\title{
Kontaktmechanische Grundlagen
}

In diesem sehr umfangreichen Kapitel sind die Methoden und Lösungen der Mechanik von axialsymmetrischen Kontaktproblemen dargelegt, die später zur Behandlung des Stoßproblems herangezogen werden. Ausgehend von der statischen Fundamentallösung der Elastizitätstheorie für einen homogenen elastischen Halbraum werden Schritt für Schritt die später im Buch verwendeten, relevanten Teile der axialsymmetrischen Kontaktmechanik entwickelt und dabei unterschiedliche physikalische Eigenschaften und Effekte (Adhäsion, Reibung, Viskoelastizität, Inhomogenität und Plastizität) berücksichtigt.

\subsection{Fundamentallösung des homogenen elastischen Halbraums}

\subsubsection{Fundamentallösung für eine Punktlast}

Eine wichtige Grundlage für viele kontaktmechanische Aufgaben ist die Fundamentallösung von Boussinesq [1, S. 81 ff.] und Cerruti [2] für die statischen Verschiebungen eines homogenen, isotropen, linear-elastischen Mediums ohne Volumenkräfte, das den unendlich ausgedehnten Halbraum $z \leq 0$ einnimmt, unter der Wirkung einer Punktlast im Koordinatenursprung mit den kartesischen Komponenten $F_{x}, F_{y}$ und $F_{z}{ }^{1}$. Die Herleitung dieser Fundamentallösung aus den Lamé-Navier-Feldgleichungen mithilfe von Methoden der Potentialtheorie ist aufwendig und soll daher an dieser Stelle nicht ausgeführt werden. Man kann sie aber bei Bedarf unter anderem bei Landau und Lifshitz [3, S. 29 ff.] nachschlagen. Man erhält folgende Ausdrücke für die kartesischen Komponenten der Verschiebungen, $u_{x}$,

\footnotetext{
${ }^{1}$ In der Kontaktmechanik führt man oft eine in den Halbraum gerichtete Koordinate $z$ ein, damit positive Normalkräfte Druckkräfte darstellen. Um Verwirrungen im Zusammenhang mit der makroskopischen Dynamik zu vermeiden, findet im vorliegenden Buch allerdings grundsätzlich die in der technischen Mechanik verbreitete Zugkraft-Konvention Anwendung.
} 
$u_{y}$ und $u_{z}$, der Oberfläche $z=0$ des elastischen Halbraums mit dem Schubmodul $G$ und der Poissonzahl $v$ :

$$
\begin{aligned}
& u_{x}(x, y, z=0)=\frac{1}{4 \pi G} \frac{1}{r}\left[-\frac{(1-2 v) x}{r} F_{z}+2(1-v) F_{x}+\frac{2 v x}{r^{2}}\left(x F_{x}+y F_{y}\right)\right], \\
& u_{y}(x, y, z=0)=\frac{1}{4 \pi G} \frac{1}{r}\left[-\frac{(1-2 v) y}{r} F_{z}+2(1-v) F_{y}+\frac{2 v y}{r^{2}}\left(x F_{x}+y F_{y}\right)\right], \\
& u_{z}(x, y, z=0)=\frac{1}{4 \pi G} \frac{1}{r}\left[2(1-v) F_{z}+\frac{1-2 v}{r}\left(x F_{x}+y F_{y}\right)\right] .
\end{aligned}
$$

Dabei (und im Folgenden) bezeichnet $r$ grundsätzlich den polaren Radius, $r=\sqrt{x^{2}+y^{2}}$. Außerdem sei angemerkt, dass die Verschiebungen und Spannungen im Inneren des Halbraums ebenfalls bestimmt werden können, aber hier aus Platzgründen weggelassen wurden.

\subsubsection{Der Kontakt zweier elastischer Körper}

Wirken zwei elastische Körper aufeinander, kann man die gerade gezeigte Fundamentallösung heranziehen, falls zwei Bedingungen erfüllt sind: Zum einen müssen die Abmaße der Körper sehr viel größer sein, als die charakteristische Länge des Kontaktgebiets, in dem sich die Körper berühren. Zum anderen müssen die Gradienten der sich berührenden Oberflächen in der Nähe des Kontaktes sehr klein sein. Diese beiden Annahmen werden sehr häufig zur sogenannten Halbraumhypothese zusammengefasst. Ist diese Hypothese erfüllt, kann man ohne Schwierigkeiten für den Kontakt der beiden elastischen Körper eine analoge Fundamentallösung formulieren wie im vorherigen Abschnitt. Wirken die beiden Körper durch eine Punktlast $\underline{F}$ im Ursprung aufeinander, ist die relative Verschiebung zweier gegenüberliegender Punkte auf den beiden Oberflächen der Körper durch

$$
\Delta \underline{u}=\underline{u}_{1}\left(x_{1}, y_{1}\right)-\underline{u}_{2}\left(x_{2}, y_{2}\right)
$$

gegeben. Unter Berücksichtigung des Wechselwirkungsprinzips

$$
\underline{F}_{1}=-\underline{F}_{2}:=\underline{F}
$$

und der jeweils entgegengesetzten Koordinatenorientierungen,

$$
\begin{aligned}
& x_{1}=-x_{2}:=x, \\
& y_{1}=-y_{2}:=y,
\end{aligned}
$$

ergeben sich damit die relativen Verschiebungen 


$$
\begin{aligned}
\Delta u_{x}(x, y) & =\frac{1}{4 \pi r}\left[-\frac{F_{z} x}{r}\left(\frac{1-2 v_{1}}{G_{1}}-\frac{1-2 v_{2}}{G_{2}}\right)+2 F_{x}\left(\frac{1-v_{1}}{G_{1}}+\frac{1-v_{2}}{G_{2}}\right)\right] \\
& +\frac{x}{2 \pi r^{3}}\left(x F_{x}+y F_{y}\right)\left(\frac{v_{1}}{G_{1}}+\frac{v_{2}}{G_{2}}\right), \\
\Delta u_{y}(x, y) & =\frac{1}{4 \pi r}\left[-\frac{F_{z} y}{r}\left(\frac{1-2 v_{1}}{G_{1}}-\frac{1-2 v_{2}}{G_{2}}\right)+2 F_{y}\left(\frac{1-v_{1}}{G_{1}}+\frac{1-v_{2}}{G_{2}}\right)\right] \\
& +\frac{y}{2 \pi r^{3}}\left(x F_{x}+y F_{y}\right)\left(\frac{v_{1}}{G_{1}}+\frac{v_{2}}{G_{2}}\right), \\
\Delta u_{z}(x, y) & =\frac{1}{4 \pi r}\left[2 F_{z}\left(\frac{1-v_{1}}{G_{1}}+\frac{1-v_{2}}{G_{2}}\right)+\frac{x F_{x}+y F_{y}}{r}\left(\frac{1-2 v_{1}}{G_{1}}-\frac{1-2 v_{2}}{G_{2}}\right)\right] .
\end{aligned}
$$

Man erkennt, dass die tangentialen Verschiebungen durch die normale Belastung - und vice versa die normalen Verschiebungen durch die tangentialen Belastungen - verschwinden, falls

$$
\frac{1-2 \nu_{1}}{G_{1}}-\frac{1-2 \nu_{2}}{G_{2}}=0 .
$$

Zwei Materialien, die diese Bedingung erfüllen, bezeichnet man als einander elastisch ähnlich.

Sind die beiden Körper elastisch ähnlich, können die oben angegebenen Beziehungen für die Komponenten der relativen Verschiebungen als Fundamentallösung eines einzigen elastischen Halbraums mit

$$
G:=\frac{G_{1} G_{2}}{G_{1}+G_{2}}
$$

und

$$
v:=\frac{v_{1} G_{2}+v_{2} G_{1}}{G_{1}+G_{2}}
$$

aufgefasst werden. Häufig führt man auch die effektiven Moduln,

$$
\begin{gathered}
\frac{1}{\tilde{E}}:=\frac{1-v}{2 G}=\frac{1-v_{1}}{2 G_{1}}+\frac{1-v_{2}}{2 G_{2}}, \\
\frac{1}{\tilde{G}}:=\frac{2-v}{4 G}=\frac{2-v_{1}}{4 G_{1}}+\frac{2-v_{2}}{4 G_{2}},
\end{gathered}
$$

ein. Das Kontaktproblem zwischen zwei elastischen Körpern kann dann auf den Kontakt eines starren Indenters mit einem elastischen Halbraum zurückgeführt werden. Dabei definiert man die Lücke zwischen den beiden kontaktierenden Oberflächen im undeformierten Zustand als Profil $f$ :

$$
f(x, y):=z_{2}(x, y)-z_{1}(x, y) .
$$


Für zwei parabolische Flächen ${ }^{2}$ mit den Krümmungsradien $R_{1}$ und $R_{2}$ ergibt sich beispielsweise

$$
f(r)=\frac{r^{2}}{2 R_{1}}+\frac{r^{2}}{2 R_{2}}=\frac{r^{2}}{2 \tilde{R}},
$$

mit dem in Gl.(2.28) eingeführten effektiven Radius $\tilde{R}$.

In den nachfolgenden Teilen dieses Buches wird, je nach der Verwendung in der jeweiligen Original-Literatur, zum Teil von dem Kontakt zwischen elastischen Körpern und an anderen Stellen von dem zwischen einem starren Indenter und einem elastischen Halbraum gesprochen. Dabei muss man sich vergegenwärtigen, dass beide Probleme im Rahmen der getroffenen Annahmen, wie gezeigt, äquivalent sind. Einen Sonderfall (und die für den weiteren Verlauf des Buches einzige relevante Ausnahme) stellt der Kontakt mit einem flachen zylindrischen Stempel dar. Da dieser unter keinen Umständen als Halbraum betrachtet werden kann, sind die im nächsten Abschnitt angegebenen Lösungen tatsächlich nur für den Kontakt eines starren Stempels mit einem elastischen Medium gültig (solange letzteres die Annahmen der Halbraumhypothese erfüllt). Die Änderungen, die sich im Fall eines elastischen Stempels ergeben, untersuchte Rao [4] in allgemeiner Form.

\subsection{Reibungsfreier Normalkontakt ohne Adhäsion}

In diesem Unterkapitel wird die allgemeine Lösung des reibungs- und adhäsionsfreien rotatiossymmetrischen Normalkontaktproblems für einfach zusammenhängende Kontaktgebiete gezeigt, die (auf verschiedenen Wegen) von Föppl [5], Schubert [6], Galin [7] und Sneddon [8] hergeleitet wurde. Ein eleganter Lösungsweg beruht auf der Idee von Mossakovski [9] und später Jäger [10], dass die Differenz zweier infinitesimal benachbarter Kontaktkonfigurationen mit den Kontaktradien $a$ und $a+\mathrm{d} a$ als eine infinitesimale Indentierung durch einen flachen zylindrischen Stempel mit dem Radius $a$ interpretiert werden kann.

\subsubsection{Lösung für den flachen zylindrischen Stempel}

Zunächst benötigt man also die von Boussinesq [1, S. 155 ff.] gefundene Lösung für das reibungsfreie elastische Normalkontaktproblem zwischen einem starren zylindrischen Stempel mit dem Radius $a$ und einem elastischen Halbraum mit dem effektiven Elastizitätsmodul $\tilde{E}$. Wird der Stempel um $d$ in den elastischen Halbraum gedrückt, sind die gemischten Randbedingungen dieses Grundproblems der Elastizitätstheorie für die Normalspannung $\sigma_{z z}$ und die vertikalen Verschiebungen $u_{z}$ durch

\footnotetext{
${ }^{2}$ Sphärische Profile können im Rahmen der Halbraumnäherung in der Nähe des Kontaktes als parabolisch angenähert werden.
} 


$$
\begin{gathered}
\sigma_{z z}(r)=0, \quad r>a, \\
u_{z}(r)=-d, \quad r \leq a,
\end{gathered}
$$

gegeben. Die Kontaktspannungen müssen außerdem grundsätzlich Druckspannungen sein.

Dieses Problem kann mithilfe der im vorherigen Unterkapitel angegebenen Fundamentallösung ohne große Schwierigkeiten vollständig gelöst werden - siehe beispielsweise die Monografien von Johnson [11, S. 59 f.] oder Popov [12, S. 313 f.]. Der Zusammenhang zwischen der Normalkraft $F_{z}$ und der Eindrucktiefe ist durch

$$
F_{z}=-2 \tilde{E} d a
$$

gegeben. Die Steifigkeit des Kontaktes ist dementsprechend

$$
k_{z}:=-\frac{\mathrm{d} F_{z}}{\mathrm{~d} d}=2 \tilde{E} a
$$

Die Spannungsverteilung im Kontakt hat die Form

$$
\sigma_{z z}(r)=-\frac{\tilde{E} d}{\pi \sqrt{a^{2}-r^{2}}}, \quad r \leq a,
$$

und die vertikalen Verschiebungen des Halbraums außerhalb des Kontaktes können zu

$$
u_{z}(r)=-\frac{2 d}{\pi} \arcsin \left(\frac{a}{r}\right), \quad r>a,
$$

bestimmt werden.

\subsubsection{Lösung für eine beliebige axialsymmetrische Indenterform}

Man betrachte nun einen rotationssymmetrischen konvexen starren Eindruckkörper mit dem Profil $f(r)$, der in einen elastischen Halbraum eingedrückt wird (siehe Abb. 3.1).

Es ist klar, dass das Kontaktgebiet kreisförmig mit dem Radius $a$ ist, wobei $a$ allerdings, im Gegensatz zum Kontakt mit einem flachen zylindrischen Stempel, nicht a priori bekannt ist,

Abb. 3.1 Indentierung eines elastischen Halbraums durch einen axialsymmetrischen starren konvexen Eindruckkörper

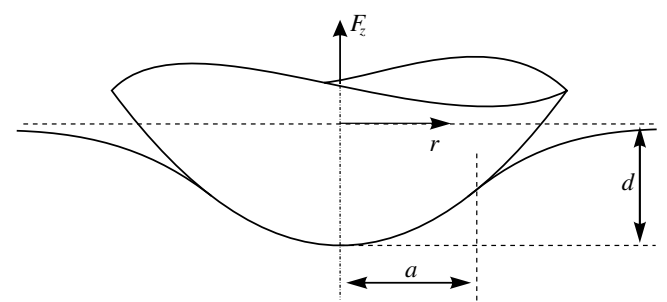


sondern im Laufe der Lösung des Kontaktproblems bestimmt werden muss. Die Bedingung (3.19) muss durch

$$
u_{z}(r)=f(r)-d, \quad r \leq a
$$

ersetzt werden, die Randbedingung (3.18) bleibt erhalten. Der Indenter habe, der Einfachheit halber und weil etwas anderes im Rahmen dieses Buches nicht gebraucht wird, keine scharfen Kanten, das Profil sei also überall stetig nach $r$ differenzierbar. Die Spannung am Rand des Kontaktes ist in diesem Fall stetig, wodurch das Randwertproblem geschlossen wird [13, S.6].

Eine Größe aus dem Tripel $\left\{a, d, F_{z}\right\}$ definiert nun eineindeutig die beiden jeweils anderen; insbesondere ist die Eindrucktiefe eine eineindeutige Funktion des Kontaktradius:

$$
d=g(a)
$$

Der Indentierungsvorgang vom Kontaktradius Null bis zum momentanen Kontaktradius $a$ kann in Anlehnung an die Idee von Mossakovski-Jäger als ein Integral von infinitesimalen Indentierungen durch flache zylindrische Stempel mit wachsenden Radien $\tilde{a}$ verstanden werden. Die gesamte Normalkraft ist durch

$$
F_{z}=\int_{0}^{a} \frac{\mathrm{d} \tilde{F}_{z}}{\mathrm{~d} \tilde{d}} \frac{\mathrm{d} \tilde{d}}{\mathrm{~d} \tilde{a}} \mathrm{~d} \tilde{a}
$$

gegeben, was man unter Verwendung der Gl.(3.21) und (3.25) nach partieller Integration ${ }^{3}$ als

$$
F_{z}=-2 \tilde{E} \int_{0}^{a}[d-g(x)] \mathrm{d} x
$$

schreiben kann. Die Druckverteilungen der Stempelindentierungen tragen zum Spannungszustand bei der radialen Koordinate $r$ erst bei, wenn der Stempelradius größer als $r$ ist. Die gesamte Spannungsverteilung ergibt sich daher zu

$$
\sigma_{z z}(r)=-\frac{\tilde{E}}{\pi} \int_{r}^{a} \frac{g^{\prime}(x)}{\sqrt{x^{2}-r^{2}}} \mathrm{~d} x, \quad r \leq a,
$$

\footnotetext{
${ }^{3}$ Zwecks der besseren Übersichtlichkeit wird die Integrationsvariable $\tilde{a}$ im Folgenden als $x$ bezeichnet. Der Sinn dieser Notation ergibt sich aus der im vierten Kapitel erläuterten Methode der Dimensionsreduktion.
} 
wobei der Hochstrich die Ableitung nach dem Argument bezeichnet. Die Verschiebungen des Halbraums sind durch

$$
u_{z}(r)=-\frac{2}{\pi} \int_{0}^{\min (r, a)} \arcsin \left(\frac{x}{r}\right) g^{\prime}(x) \mathrm{d} x-\int_{\max (r, a)}^{a} g^{\prime}(x) \mathrm{d} x
$$

gegeben, was sich wiederum nach partieller Integration zu

$$
u_{z}(r)=-\frac{2}{\pi} \int_{0}^{\min (r, a)} \frac{d-g(x)}{\sqrt{r^{2}-x^{2}}} \mathrm{~d} x
$$

vereinfachen lässt. Aus der Äquivalenz von (3.24) und (3.30) innerhalb des Kontaktgebiets folgt:

$$
f(r)=\frac{2}{\pi} \int_{0}^{r} \frac{g(x)}{\sqrt{r^{2}-x^{2}}} \mathrm{~d} x
$$

Dies ist eine Abeltransformation, die man mit dem Ergebnis [14, S. 353]

$$
g(x)=\frac{\mathrm{d}}{\mathrm{d} x}\left[\int_{0}^{|x|} \frac{r f(r)}{\sqrt{x^{2}-r^{2}}} \mathrm{~d} r\right]=|x| \int_{0}^{|x|} \frac{f^{\prime}(r)}{\sqrt{x^{2}-r^{2}}} \mathrm{~d} r
$$

invertieren kann. Die Gl.(3.25), (3.27), (3.28), (3.30) und (3.32) reproduzieren die Lösung des rotationssymmetrischen Boussinesq-Problems von Föppl-Schubert-Galin-Sneddon.

Für den Normalkontakt zwischen einer starren Kugel mit dem Radius $\tilde{R}$ und einem elastischen Halbraum erhält man im Rahmen der Halbraumnäherung $a \ll \tilde{R}$ das parabolische Profil

$$
f(r) \approx \frac{r^{2}}{2 \tilde{R}}
$$

Die Lösung in der parabolischen Näherung geht auf die klassische Arbeit von Hertz [15] zurück, die exakte Kugelform wurde von Segedin [16] zuerst behandelt. Allerdings unterscheidet sich die Lösung der Kugel selbst für Werte $\tilde{R} \approx 4 a$, die die Halbraumhypothese bereits grob verletzen, nur sehr geringfügig von der des Paraboloids [17, S. 21 f.]. Setzt man die parabolische Näherung (3.33) in die oben abgeleiteten Gleichungen der allgemeinen Lösung ein, findet man die Beziehungen 


$$
\begin{aligned}
g(x) & =\frac{x^{2}}{\tilde{R}}, \\
d(a) & =\frac{a^{2}}{\tilde{R}}, \\
F_{z}(a) & =-\frac{4}{3} \tilde{E} \frac{a^{3}}{\tilde{R}}, \\
\sigma_{z z}(r) & =-\frac{2 \tilde{E}}{\pi \tilde{R}} \sqrt{a^{2}-r^{2}}, \quad r \leq a,
\end{aligned}
$$

durch die das Kontaktproblem vollständig gelöst wird.

Für ein Profil in der Form eines Potenzgesetzes (dies schließt unter anderem die Spezialfälle des konischen und des oben gezeigten parabolischen Kontaktes mit ein),

$$
f(r)=A r^{n}, \quad n \in \mathbb{R}^{+},
$$

mit einer positiven Konstante $A$, lautet die Lösung des Kontaktproblems für die makroskopischen Größen wie folgt:

$$
\begin{aligned}
d(a) & =\beta(n) A a^{n}, \quad \beta(n):=\sqrt{\pi} \frac{\Gamma(n / 2+1)}{\Gamma[(n+1) / 2]}, \\
F_{z}(a) & =-\frac{2 n}{n+1} \tilde{E} \beta(n) A a^{n+1} .
\end{aligned}
$$

Dabei bezeichnet $\Gamma$ die im Anhang definierte Gamma-Funktion. Für $n=2$ und $A=1 /(2 \tilde{R})$ ergeben sich natürlich die obigen Ergebnisse des parabolischen Profils. Auf die Angabe der lokalen Spannungen wurde verzichtet. Es sei aber in diesem Zusammenhang auf das Handbuch von Popov et al. [17, S. 28] verwiesen.

\subsubsection{Einfluss des Reibregimes}

Bisher wurde nur der reibungsfreie Normalkontakt behandelt, bei dem mögliche relative radiale Verschiebungen zwischen den kontaktierenden Oberflächen zu keinen zusätzlichen radialen Spannungen an der Oberfläche des Halbraum-Mediums führen. Es sind aber natürlich auch andere Regime denkbar, z. B. Kontakte ohne Gleiten (also mit einem unendlichen Reibbeiwert) oder solche mit einem endlichen Reibungskoeffizienten. Die im vorangegangenen Abschnitt verwendete Methode der Superposition von infinitesimalen Flachstempellösungen ist auch auf solche Kontakte anwendbar ${ }^{4}$, allerdings sind die Lösungen deutlich komplizierter (und im Fall endlicher Reibung auch nur noch numerisch möglich), da bereits

\footnotetext{
${ }^{4}$ Tatsächlich stammt die Idee von Mossakovski ursprünglich aus der Behandlung des Normalkontaktproblems ohne Gleiten.
} 
die Grundaufgabe der Indentierung durch den flachen zylindrischen Stempel mathematisch sehr viel komplexer ist als im reibungsfreien Fall.

Andererseits gibt es im Fall elastisch ähnlicher Kontaktpartner gar keine relativen radialen Verschiebungen bei der Normalindentierung und das Reibregime spielt daher für Kontakte elastisch ähnlicher Materialien keine Rolle. Dieser Fall der elastischen Ähnlichkeit ist der hauptsächlich in dem vorliegenden Buch untersuchte, deswegen soll der Normalkontakt mit Reibung an dieser Stelle nicht ausführlich betrachtet werden. Es sei aber auf die zentralen Publikationen von Mossakovski [9, 18] und Spence [19] zum Normalkontakt ohne Gleiten und von Spence [20], Storåkers und Elaguine [21] und Zhupanska [22] zum Normalkontakt mit endlicher Reibung verwiesen. Eine gute Übersicht über die existierende Literatur zu diesem Thema liefern außerdem die Arbeit von Borodich und Keer [23] und das Handbuch von Popov et al. [17, S. 51 ff.]. Für Materialien mit positiven Poissonzahlen unterscheiden sich die Werte der Kontaktsteifigkeit für den reibungsfreien Kontakt und den Kontakt ohne Gleiten nur um maximal 10\% [17, S. 55], der reibungsfreie Fall kann daher oft auch dann als sehr gute Näherung ${ }^{5}$ herangezogen werden, wenn seine formalen Voraussetzungen nicht exakt erfüllt sind.

\subsection{Reibungsfreier Normalkontakt mit Adhäsion}

\subsubsection{Einführung}

Die Oberflächen fester, elektrisch neutraler Körper wechselwirken durch relativ schwache und schnell mit dem Abstand fallende, in der Regel anziehende Kräfte, die man als Adhäsionskräfte bezeichnet. Die physikalische Natur dieser Kräfte kann verschiedener Art sein, ihr Ursprung sind dabei van-der-Waals- oder andere schwache molekulare Wechselwirkungen. Je nach der Form der Wechselwirkung lässt sich zwischen den Oberflächen der Körper ein mikroskopisches Potential $\sigma_{\text {adh }}$ als Funktion des Abstands $h>0$ einführen, das eine charakteristische Reichweite $h_{1}$ und einen Gleichgewichtsabstand $h_{0}$ von der gleichen Größenordnung besitzt. Die Arbeit pro Fläche, die nötig ist, um zwei Oberflächen gegen die Wirkung dieses Potentials aus dem Gleichgewichtsabstand vollständig voneinander zu trennen, sei als $\Delta \gamma$ bezeichnet,

$$
\Delta \gamma:=\int_{h_{0}}^{\infty} \sigma_{\mathrm{adh}}(h) \mathrm{d} h .
$$

Die Adhäsion als Oberflächen- und die Elastizität als Volumen-Wechselwirkung skalieren unterschiedlich. Daher spielt die Adhäsion bei kontaktmechanischen Problemen vorwiegend in besonders kleinen Systemen (der Mikro- oder Nanoskala) eine Rolle. Sie gewinnt

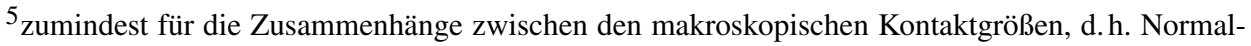
kraft, Kontaktradius und Eindrucktiefe
} 
aber auch an Bedeutung, wenn einer der Kontaktpartner sehr weich ist oder die beteiligten Oberflächen sehr glatt sind.

Falls die charakteristische Reichweite der Adhäsion vernachlässigbar klein gegenüber der kleinsten Skala des jeweiligen Kontaktproblems ist, meistens der Indentierungstiefe, spielt die konkrete Form des Potentials keine Rolle und die einzige notwendige Größe zur Charakterisierung der adhäsiven Wechselwirkung ist $\Delta \gamma$. Es reicht dann eine energetische Betrachtung zur Behandlung der Oberflächenenergie aus, wie zuerst im Rahmen der Linear-Elastischen Bruchmechanik in der klassischen Arbeit von Griffith [24] gezeigt wurde. Mit dem gleichen Ansatz wie Griffith lösten 50 Jahre später Johnson, Kendall und Roberts (JKR, [25]) das adhäsive Normalkontaktproblem von Kugeln im Grenzfall vernachlässigbarer Reichweite der adhäsiven Wechselwirkung. Der entgegengesetzte Fall, dass diese Reichweite sehr viel größer als die der elastischen Wechselwirkung ist, wurde für den Normalkontakt von Kugeln wenig später von Derjaguin, Muller und Toporov (DMT, [26]) behandelt. Sie nahmen an, dass dann die elastischen Spannungen im Kontakt, wiederum unabhängig von der konkreten Form des adhäsiven Potentials, durch die Adhäsion nicht beeinflusst werden und der bekannten Hertzschen Verteilung (3.37) genügen.

Die JKR- und die DMT-Theorie unterscheiden sich in ihren Vorhersagen. So erhalten sie für die maximale adhäsive Zugkraft $F_{A}$, die der Kontakt aufbringen kann, die verschiedenen Werte

$$
\begin{aligned}
F_{A}^{\mathrm{JKR}} & =\frac{3}{2} \pi \Delta \gamma \tilde{R}, \\
F_{A}^{\mathrm{DMT}} & =2 \pi \Delta \gamma \tilde{R} .
\end{aligned}
$$

Im Rahmen der DMT-Theorie ist der Kontakt in diesem Zustand bereits auf einen Punkt zusammengeschrumpft, das Ergebnis (3.43) stimmt daher mit dem von Bradley [27] für den Kontakt starrer Kugeln überein. Tabor [28] konnte zeigen, dass beide Theorien korrekte Grenzfälle beschreiben und dass das Verhalten im Übergangsbereich nur von dem Verhältnis zwischen dem Gleichgewichtsabstand $h_{0}$ und der charakteristischen Länge $\Delta l$ des adhäsiven Halses,

$$
\Delta l \approx\left(\frac{\tilde{R} \Delta \gamma^{2}}{\tilde{E}^{2}}\right)^{1 / 3}
$$

bestimmt wird. Der Tabor-Parameter zur Berücksichtigung der Reichweite der Adhäsion ist also

$$
\lambda_{T}=\frac{1}{h_{0}}\left(\frac{\tilde{R} \Delta \gamma^{2}}{\tilde{E}^{2}}\right)^{1 / 3}
$$

Der genaue Übergang zwischen den beiden Grenzfällen hängt natürlich - im Gegensatz zu den Grenzfällen selbst - von der konkreten Form des Potentials ab. Die erste rigorose 
analytische Lösung für den adhäsiven Normalkontakt von Kugeln bei einem beliebigen Tabor-Parameter gelang dabei Maugis [29]. Dieser verwendete das theoretische Potential einer konstanten Adhäsionsspannung innerhalb einer festen Reichweite,

$$
\sigma_{\mathrm{adh}}(h)=\sigma_{m} \mathrm{H}\left(h_{1}-h\right),
$$

mit der Heaviside-Stufenfunktion $\mathrm{H}(\cdot)$, das zuerst im Rahmen der Bruchmechanik von Dugdale [30] benutzt wurde und streng genommen keine physikalische Realität besitzt. Sein großer Vorteil besteht aber darin, dass es - im Gegensatz zu physikalisch rigoroseren Potentialen - eine analytische Einbindung in die Kontaktmechanik erlaubt. Außerdem ist das qualitative Verhalten sicher unabhängig von der Form des Potentials; zur allgemeinen Untersuchung des Einflusses des Tabor-Parameters in adhäsiven Kontakten ist daher die klassische Theorie von Maugis ein sehr berechtigter Ansatz. Trotzdem wurden auch andere Ansätze verfolgt. Greenwood [31] und Feng [32] untersuchten beispielsweise in ausführlichen numerischen Studien das Potential

$$
\sigma_{\mathrm{adh}}(h)=\frac{8 \Delta \gamma}{3 h_{0}}\left[\left(\frac{h_{0}}{h}\right)^{3}-\left(\frac{h_{0}}{h}\right)^{9}\right],
$$

das von Muller et al. [33] aus dem Lennard-Jones-(12,6)-Potential zwischen zwei Molekülen hergeleitet wurde. Damit das Maximum dieser Funktion mit dem Wert $\sigma_{m}$ aus dem Dugdale-Potential übereinstimmt und die Oberflächentrennungsarbeit $\Delta \gamma$ für beide Potentiale den gleichen Wert annimmt, muss das Verhältnis zwischen der Reichweite des DugdalePotentials und dem Gleichgewichtsabstand des Lennard-Jones-Potentials zu

$$
\frac{h_{1}}{h_{0}}=\frac{9 \sqrt{3}}{16} \approx 0,97
$$

gewählt werden. Für diesen Fall sind die beiden Potentiale in normierter Darstellung (in ihrem Zugbereich) in Abb. 3.2 gezeigt.

Greenwood und Johnson [34] konstruierten ein künstliches (und etwas merkwürdiges) Potential mit dem Zweck, die resultierende Kontaktmechanik so weit wie möglich

Abb.3.2 Der aus dem Lennard-Jones-(12,6)-Potential abgeleitete Ausdruck (3.47) und das Dugdale-Potential (3.46) für die adhäsive Spannung, jeweils in normierter Darstellung

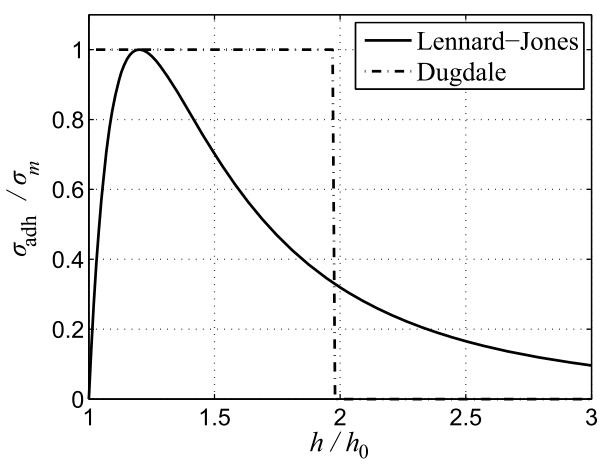


zu vereinfachen. Auch ihre Theorie enthält natürlich die JKR- und die DMT-Theorie als Grenzfälle. Eine von der genauen Form des Wechselwirkungspotentials unabhängige Beschreibung des adhäsiven Normalkontaktes von Kugeln wurde außerdem von Barthel [35] gegeben. Einen sehr guten Überblick über die Rolle der Adhäsion in der Kontaktmechanik (besonders im Hinblick auf die Kontaktmechanik rauer Oberflächen) bietet schließlich die kürzlich erschienene Arbeit von Ciavarella et al. [36].

In diesem Buch finden später bei der genaueren Untersuchung des Normalstoßproblems die JKR-Theorie und die Theorie von Maugis Anwendung, die daher in den beiden folgenden Abschnitten noch einmal detaillierter dargestellt werden.

\subsubsection{Adhäsiver Normalkontakt in der JKR-Näherung}

Im Rahmen der JKR-Theorie hat die Adhäsion die Reichweite Null, die adhäsiven Spannungen wirken also nur in der Fläche des direkten Kontaktes. Damit sind die Randbedingungen des axialsymmetrischen Normalkontaktproblems die gleichen wie im Fall des nicht-adhäsiven Kontaktes: außerhalb des Kontaktes gibt es keine Spannungen und innerhalb des Kontaktes wird die Verschiebung durch die Form des Eindruckkörpers vorgegeben. Nur der Zusammenhang (3.25) zwischen der Eindrucktiefe und dem Kontaktradius verliert seine Gültigkeit durch die Bildung des adhäsiven Halses. Aus der Gleichheit der Randbedingungen folgt sofort, dass das adhäsive Kontaktproblem aus der Superposition des nicht-adhäsiven Problems mit einer Starrkörperverschiebung des Kontaktgebiets, d.h. mit einer Indentierung durch einen flachen zylindrischen Stempel mit dem Radius $a$, hervorgeht. Dies ist die zentrale Idee der JKR-Theorie, aus der sich die Lösung des Kontaktproblems ohne Schwierigkeiten bestimmen lässt, da die Lösungen des nicht-adhäsiven Kontaktes für einen beliebigen axialsymmetrischen Indenter und den Flachstempel bereits bekannt sind. Nur die zusätzliche Starrkörperverschiebung $\Delta l$ muss bestimmt werden. Dies gelingt aber leicht, indem, in Analogie zum Griffith-Kriterium, der Gleichgewichtszustand über das Minimum der Gesamtenergie ermittelt wird. Im Folgenden wird mithilfe der obigen Überlegungen das axialsymmetrische reibungsfreie adhäsive Normalkontaktproblem in der JKR-Näherung gelöst. Die Lösung wurde, auf eine etwas andere Art hergeleitet, zuerst von Barquins und Maugis [37] präsentiert. Mit den gleichen Methoden können außerdem auch allgemeine adhäsive Normalkontakte in der JKR-Näherung behandelt werden [38-41].

Zunächst wird der Indenter ohne Berücksichtigung der Adhäsion bis zum Kontaktradius $a$ in den elastischen Halbraum eingedrückt. Die Werte der Normalkraft, der Indentierungstiefe, der Kontaktsteifigkeit und der gespeicherten elastischen Energie sind aus dem vorherigen Unterkapitel bekannt. Um Verwechslungen vorzubeugen, kennzeichnet im Folgenden das Superskript „n.a.“ die nicht-adhäsiven Größen. Anschließend wird das gesamte Kontaktgebiet auf

$$
d=d^{\text {n.a. }}-\Delta l
$$


angehoben, die Normalkraft ist dann

$$
F_{z}=F_{z}^{\text {n.a. }}+k_{z}^{\text {n.a. }} \Delta l
$$

Die gesamte Energie des Systems am Schluss des Vorgangs beträgt (unter Berücksichtigung der Oberflächenenergie)

$$
U_{\mathrm{tot}}=U_{\mathrm{el}}^{\text {n.a. }}+\frac{k_{z}^{\text {n.a. }}}{2} \Delta l^{2}+F_{z}^{\text {n.a. }} \Delta l-\pi a^{2} \Delta \gamma
$$

Bei vorgegebener (konstanter) Eindrucktiefe $d$ ist

$$
\frac{\partial \Delta l}{\partial a}=\frac{\partial d^{\text {n.a. }}}{\partial a}
$$

Der Kontaktradius stellt sich so ein, dass die Gesamtenergie des Systems minimal ist. Das führt auf die notwendige Bedingung

$$
\frac{\partial U_{\text {tot }}}{\partial a}=-F_{z}^{\text {n.a. }} \frac{\partial d^{\text {n.a. }}}{\partial a}+\frac{\Delta l^{2}}{2} \frac{\partial k_{z}^{\text {n.a. }}}{\partial a}+k_{z}^{\text {n.a. }} \Delta l \frac{\partial \Delta l}{\partial a}-k_{z}^{\text {n.a. }} \cdot \frac{\partial d^{\text {n.a. }}}{\partial a} \Delta l+F_{z}^{\text {n.a. }} \frac{\partial \Delta l}{\partial a}-2 \pi a \Delta \gamma=0,
$$

die bei konstanter Eindrucktiefe auf die Beziehung

$$
\Delta l= \pm \sqrt{\frac{4 \pi a \Delta \gamma}{\partial k_{z}^{\text {n.a. }} / \partial a}}= \pm \sqrt{\frac{2 \pi a \Delta \gamma}{\tilde{E}}}
$$

führt. Dabei entspricht der positive Wert einem Minimum der Gesamtenergie.

Die Lösung des parabolischen Kontaktes ist mit den Ergebnissen aus dem vorherigen Unterkapitel 3.2 damit durch

$$
\begin{aligned}
d(a) & =\frac{a^{2}}{\tilde{R}}-\sqrt{\frac{2 \pi a \Delta \gamma}{\tilde{E}}}, \\
F_{z}(a) & =-\frac{4}{3} \tilde{E} \frac{a^{3}}{\tilde{R}}+\sqrt{8 \pi a^{3} \Delta \gamma \tilde{E}},
\end{aligned}
$$

gegeben.

Mit der Bedingung (3.53) wurde ein Gleichgewichtszustand gefunden, über die Stabilität dieses Gleichgewichts aber noch keine Aussage getroffen. Wird die Kugel ohne Kontakt auf den Halbraum zubewegt, gibt es im Zustand der ersten Berührung, $d=0$, zwei Lösungen der Gl. (3.55) für den Kontaktradius, von denen allerdings, wie man sich leicht überzeugt, nur

$$
a_{0}=\left(\frac{2 \pi \Delta \gamma \tilde{R}^{2}}{\tilde{E}}\right)^{1 / 3}
$$


stabil ist. Es bildet sich also spontan ein Kontaktgebiet mit dem Radius $a_{0}$ aus. Zieht man die Kugel von dem Halbraum ab, haftet sie solange daran, bis der Kontakt seine Stabilität verliert und sich spontan auflöst. In welcher Kontaktkonfiguration das passiert, hängt von der Apparatur ab, mit der die Kugel angehoben wird. Diese Apparatur habe eine Normalsteifigkeit $\hat{k}_{z}$. Die Stabilitätsgrenze ergibt sich aus der Bedingung,

$$
\hat{k}_{z}=\frac{\mathrm{d} F_{z}}{\mathrm{~d} d}=\frac{\mathrm{d} F_{z}}{\mathrm{~d} a} \frac{\mathrm{d} a}{\mathrm{~d} d} .
$$

Besonders einfach wird dieses Kriterium in zwei Spezialfällen, der Weg-, beziehungsweise Kraftsteuerung. Für weggesteuerte Versuche ist die Apparatur unendlich steif, die Bedingung (3.58) vereinfacht sich somit zu der Bedingung

$$
\frac{\mathrm{d} d}{\mathrm{~d} a}=0 .
$$

Die entsprechenden kritischen Werte des Kontaktradius, der Eindrucktiefe und der Normalkraft sind in diesem Fall (gekennzeichnet durch das Superskript „WS“) durch

$$
a_{c}^{\mathrm{WS}}=\left(\frac{\pi \tilde{R}^{2} \Delta \gamma}{8 \tilde{E}}\right)^{1 / 3}, \quad d_{c}^{\mathrm{WS}}=-\frac{3}{4}\left(\frac{\pi^{2} \Delta \gamma^{2} \tilde{R}}{\tilde{E}^{2}}\right)^{1 / 3}, \quad F_{z, c}^{\mathrm{WS}}=\frac{5}{6} \pi \Delta \gamma \tilde{R}
$$

gegeben. Bei ideal-kraftgesteuerten Versuchen ist die Apparatur unendlich weich, das Stabilitätskriterium ist dann

$$
\frac{\mathrm{d} F_{z}}{\mathrm{~d} a}=0
$$

Die entsprechenden kritischen Werte des Kontaktradius, der Eindrucktiefe und der Normalkraft sind

$$
a_{c}^{\mathrm{KS}}=\left(\frac{9 \pi \tilde{R}^{2} \Delta \gamma}{8 \tilde{E}}\right)^{1 / 3}, \quad d_{c}^{\mathrm{KS}}=-\frac{1}{4}\left(\frac{3 \pi^{2} \Delta \gamma^{2} \tilde{R}}{\tilde{E}^{2}}\right)^{1 / 3}, \quad F_{z, c}^{\mathrm{KS}}=\frac{3}{2} \pi \Delta \gamma \tilde{R} .
$$

Die letzte Relation entspricht der bereits in Gl. (3.42) eingeführten maximalen Adhäsionskraft in der JKR-Näherung. Normiert man alle Größen auf ihre kritischen Werte bei Kraftsteuerung, erhält man die Zusammenhänge

$$
\begin{aligned}
\frac{d}{\left|d_{c}^{\mathrm{KS}}\right|} & =3\left(\frac{a}{a_{c}^{\mathrm{KS}}}\right)^{2}-4 \sqrt{\frac{a}{a_{c}^{\mathrm{KS}}}}, \\
\frac{F_{z}}{F_{c}^{\mathrm{KS}}} & =-\left(\frac{a}{a_{c}^{\mathrm{KS}}}\right)^{3}+2 \sqrt{\left(\frac{a}{a_{c}^{\mathrm{KS}}}\right)^{3}} .
\end{aligned}
$$

Diese sind in Abb. 3.3 dargestellt. 
Abb.3.3 Normalkraft und Indentierungstiefe als Funktionen des Kontaktradius in normierten Größen für den adhäsiven Normalkontakt von Kugeln in der JKR-Näherung

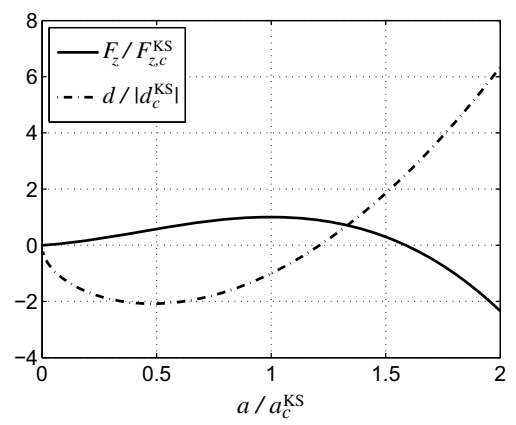

Abb. 3.4 zeigt die dadurch implizit definierte Relation zwischen den normierten Werten der Normalkraft und der Indentierungstiefe. Der durchgezogene Teil ist dabei unabhängig von der Versuchsapparatur stabil. Die Stabilität in dem strichpunktierten Teil hängt von der Steifigkeit der Apparatur ab, der punktierte Teil ist grundsätzlich instabil.

Falls das Profil $f(r)$ in der Form eines Potenzgesetzes geschrieben werden kann - siehe Gl. (3.38) - sind die Zusammenhänge zwischen Normalkraft, Kontaktradius und Eindrucktiefe mithilfe der nicht-adhäsiven Lösung aus den Gl. (3.39) und (3.40) durch

$$
\begin{aligned}
d(a) & =\beta(n) A a^{n}-\sqrt{\frac{2 \pi a \Delta \gamma}{\tilde{E}}}, \\
F_{z}(a) & =-\frac{2 n}{n+1} \tilde{E} \beta(n) A a^{n+1}+\sqrt{8 \pi a^{3} \Delta \gamma \tilde{E}}
\end{aligned}
$$

gegeben. Für den weiteren Verlauf dieses Buches sind nur die kritischen Größen im weggesteuerten Fall von Bedeutung. Dabei ist $n=1 / 2$ ein kritischer Fall, er trennt zwei unterschiedliche Formen von Instabilitäten: für $n>1 / 2$ die instabile Auflösung des Kontaktes und für $n<1 / 2$ die instabile (unbegrenzte) Ausbreitung des Kontaktes [42]. Es sollen daher im Folgenden für JKR-adhäsive Normalkontakte nur Profile mit $n>1 / 2$ betrachtet

Abb. 3.4 Normalkraft als

Funktion der

Indentierungstiefe; der gepunktete Teil ist instabil, der strichpunktierte stabil oder instabil je nach der Art der Versuchssteuerung, der durchgezogene Teil ist stabil

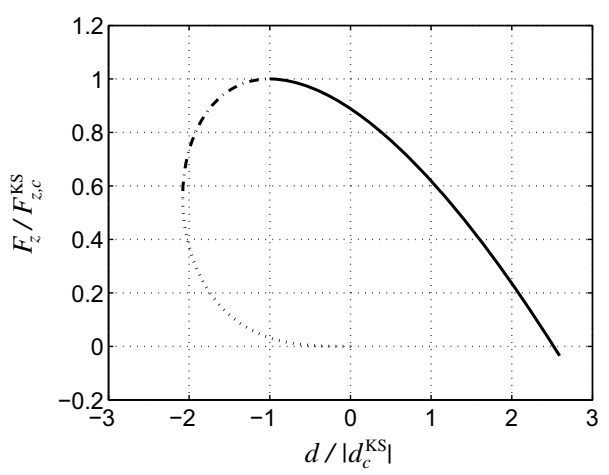


werden. Man erhält für den kritischen Kontaktradius bei Wegsteuerung auf die gleiche Art und Weise wie oben

$$
a_{c}^{\mathrm{WS}}=\left(\frac{\pi \Delta \gamma}{2 \tilde{E}}\right)^{\frac{1}{2 n-1}}\left(\frac{1}{n \beta(n) A}\right)^{\frac{2}{2 n-1}}
$$

und für die entsprechenden Werte der Eindrucktiefe und der Normalkraft

$$
\begin{aligned}
& d_{c}^{\mathrm{WS}}=\left(\frac{1}{n}-2\right)\left(\frac{\pi \Delta \gamma}{2 \tilde{E}}\right)^{\frac{n}{2 n-1}}\left(\frac{1}{n \beta(n) A}\right)^{\frac{1}{2 n-1}}, \\
& F_{c}^{\mathrm{WS}}=\left(4-\frac{2}{n+1}\right) \tilde{E}^{\frac{n-2}{2 n-1}}\left(\frac{\pi \Delta \gamma}{2}\right)^{\frac{n+1}{2 n-1}}\left(\frac{1}{n \beta(n) A}\right)^{\frac{3}{2 n-1}} .
\end{aligned}
$$

\subsubsection{Theorie von Maugis (parabolischer Kontakt)}

Der Einfluss der Reichweite der Adhäsion auf das Normalstoßproblem wird später zur Veranschaulichung der relevanten Mechanismen nur für den Kontakt von Kugeln genauer untersucht, da der allgemeine axialsymmetrische Fall für dieses Problem sehr unübersichtlich ist. Im Gegensatz zu den vorhergehenden Abschnitten soll an dieser Stelle daher ausschließlich der parabolische Kontakt betrachtet werden.

Der Übergang von der JKR- zur DMT-Theorie für den parabolischen Kontakt wurde von Maugis [29] vollständig analytisch mithilfe des Dugdale-Modells (3.46) für das adhäsive Potential gefunden. Neben dem Gebiet des direkten Kontaktes $r \leq a$ gibt es in diesem Kontaktproblem das ringförmige Gebiet $a<r \leq b$, in dem nur die adhäsive Zugspannung auf die beiden Oberflächen wirkt. Das Randwertproblem wird außerdem durch die Forderungen geschlossen, dass die Spannung am Rand des direkten Kontaktes stetig ist und der Abstand zwischen den Oberflächen bei $r=b$ der Reichweite der Adhäsion entspricht (siehe Abb. 3.5):

$$
h_{1}=\frac{b^{2}}{2 \tilde{R}}-d-u_{z}(b)
$$

\footnotetext{
Abb.3.5 Schematische

Darstellung des

Maugis-adhäsiven

Normalkontaktes zwischen einem starren Paraboloid und einem elastischen Halbraum; Veranschaulichung aller relevanten Längen
}

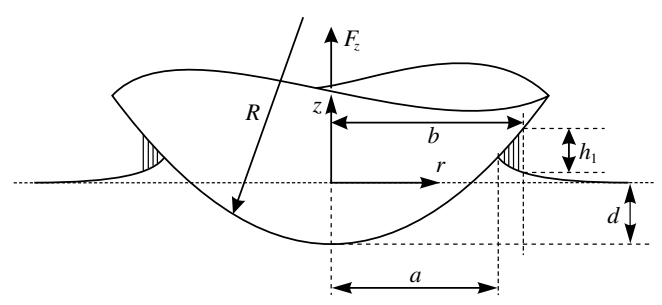


Maugis löste diese Aufgabe mit Methoden der Linear-Elastischen Bruchmechanik, indem er einen äußeren Riss unter Einfluss der Dugdale-Spannung betrachtete ${ }^{6}$. Die Herleitung von Maugis ist umfangreich und da im nächsten Kapitel eine deutlich einfachere Herleitung mithilfe der Methode der Dimensionsreduktion gezeigt ist, wird an dieser Stelle auf die Darstellung des Lösungswegs von Maugis verzichtet. Man erhält für die Spannungsverteilung im Gebiet des direkten Kontaktes

$$
\sigma_{z z}(r)=-\frac{2 \tilde{E}}{\pi \tilde{R}} \sqrt{a^{2}-r^{2}}+\frac{2 \sigma_{m}}{\pi} \arctan \left(\frac{\sqrt{b^{2}-a^{2}}}{\sqrt{a^{2}-r^{2}}}\right), \quad r \leq a .
$$

Der Zusammenhang zwischen der Eindrucktiefe $d$ und den beiden Kontaktradien $a$ und $b$ ist

$$
d=\frac{a^{2}}{\tilde{R}}-\frac{2 \sigma_{m}}{\tilde{E}} \sqrt{b^{2}-a^{2}}
$$

und die gesamte Normalkraft beträgt

$$
F_{z}=-\frac{4}{3} \tilde{E} \frac{a^{3}}{\tilde{R}}+2 \sigma_{m}\left[b^{2} \arccos \left(\frac{a}{b}\right)+a \sqrt{b^{2}-a^{2}}\right] .
$$

Außerdem führt die Forderung (3.70) auf die Gleichung

$$
\frac{\pi h_{1}}{2}=\arccos \left(\frac{a}{b}\right)\left(\frac{b^{2}}{2 \tilde{R}}-d\right)+\frac{a}{2 \tilde{R}} \sqrt{b^{2}-a^{2}}-\frac{2 \sigma_{m}}{\tilde{E}}(b-a) .
$$

Normiert man alle Größen auf die in den Gl. (3.62) gegebenen kritischen Werte in der JKR-Näherung und führt außerdem die Kürzel

$$
m:=\frac{b}{a}, \quad \Lambda:=\frac{\left|d_{c}^{\mathrm{KS}}\right|}{h_{1}}
$$

ein, können die Gl. (3.72), (3.73) und (3.74) wie folgt in dimensionsfreier Form geschrieben werden:

$$
\begin{aligned}
\hat{d} & =3 \hat{a}^{2}-\frac{16}{\pi} \hat{a} \Lambda \sqrt{m^{2}-1}, \\
\hat{F}_{z} & =-\hat{a}^{3}+\frac{4}{\pi} \Lambda \hat{a}^{2}\left[m^{2} \arccos \left(\frac{1}{m}\right)+\sqrt{m^{2}-1}\right], \\
1 & =\frac{32}{\pi^{2}} \Lambda^{2} \hat{a}\left[\arccos \left(\frac{1}{m}\right) \sqrt{m^{2}-1}-m+1\right]+ \\
& +\frac{3 \Lambda \hat{a}^{2}}{\pi}\left[\left(m^{2}-2\right) \arccos \left(\frac{1}{m}\right)+\sqrt{m^{2}-1}\right] .
\end{aligned}
$$

\footnotetext{
${ }^{6}$ Der Rand des direkten Kontaktes stellt die Spitze eines externen axialsymmetrischen Risses dar; für eine ausführliche Diskussion der Parallelen zwischen Kontakt- und Bruchmechanik sei auf die Arbeit von Giannakopoulos et al. [43] verwiesen.
} 
Der Parameter $\Lambda$ in Gl. (3.75) ist die Version des Tabor-Parameters für das DugdalePotential. Unter der Annahme (3.48) lässt er sich durch $\Lambda=4 \pi^{\frac{2}{3}} 3^{-\frac{13}{6}} \lambda_{T} \approx 0,79 \lambda_{T}$ in den klassischen Tabor-Parameter des Lennard-Jones-Potentials umrechnen.

Der JKR-Grenzfall entspricht $\Lambda \rightarrow \infty$, wobei $m \rightarrow 1$. Dann dominiert in Gl.(3.78) der erste Summand und es ergibt sich der Grenzübergang

$$
\frac{4}{\pi} \Lambda \sqrt{m^{2}-1} \rightarrow \frac{1}{\sqrt{\hat{a}}} .
$$

Der DMT-Grenzfall entspricht $\Lambda \rightarrow 0$, wobei $m \rightarrow \infty$. Dann dominiert in Gl.(3.78) der zweite Summand und man erhält den Grenzübergang

$$
\Lambda(\hat{a} m)^{2} \rightarrow \frac{2}{3} .
$$

Es sind auch Konfigurationen ohne direkten Kontakt möglich. In diesem Fall lauten die Beziehungen zwischen den makroskopischen Kontaktgrößen und der Kontaktkonfiguration in entdimensionierter Form wie folgt:

$$
\begin{aligned}
\hat{d} & \leq-\frac{16}{\pi} \Lambda \hat{b} \quad \text { (nicht unbedingt eine Gleichung), } \\
\hat{F}_{z} & =2 \Lambda \hat{b}^{2} \\
1 & =\Lambda\left(\frac{3}{2} \hat{b}^{2}-\hat{d}\right)-\frac{32}{\pi^{2}} \Lambda^{2} \hat{b} \quad \text { (trotzdem immer eine Gleichung) }
\end{aligned}
$$

wobei die Größe $\hat{b}:=m \hat{a}$ eingeführt wurde. Die oben abgeleiteten (bzw. implizit definierten) Zusammenhänge zwischen der Normalkraft und der Eindrucktiefe sind in den normierten Variablen in Abb. 3.6 gezeigt. Wenn der Zustand ohne direkten Kontakt gerade erreicht wird, wird aus der Ungleichung (3.81) eine Gleichung und man erhält die entsprechenden Werte der normierten Eindrucktiefe und des normierten Radius der adhäsiven Zone:

$$
\begin{aligned}
& \hat{b}_{a 0}=\frac{16 \Lambda}{3 \pi^{2}}\left[2-\pi+\sqrt{(2-\pi)^{2}+\frac{3 \pi^{4}}{2^{7} \Lambda^{3}}}\right], \\
& \hat{d}_{a 0}=-\frac{16}{\pi} \Lambda \hat{b}_{a 0} .
\end{aligned}
$$

Die analytische Bestimmung der kritischen Kontaktkonfigurationen in der Theorie von Maugis ist sehr aufwändig. Totale Differentiation der Gl.(3.76) bis (3.78) liefert 


$$
\begin{aligned}
\mathrm{d} \hat{d} & =\left(6 \hat{a}-\frac{16}{\pi} \Lambda \sqrt{m^{2}-1}\right) \mathrm{d} \hat{a}-\frac{16}{\pi} \hat{a} \Lambda \frac{m}{\sqrt{m^{2}-1}} \mathrm{~d} m \\
\mathrm{~d} \hat{F}_{z} & =\left[-3 \hat{a}^{2}+\frac{8}{\pi} \Lambda \hat{a}\left(m^{2} \arccos \left(\frac{1}{m}\right)+\sqrt{m^{2}-1}\right)\right] \mathrm{d} \hat{a}+ \\
& +\frac{8}{\pi} \Lambda \hat{a}^{2} m\left[\frac{1}{\sqrt{m^{2}-1}}+\arccos \left(\frac{1}{m}\right)\right] \mathrm{d} m \\
0 & =\frac{16 \Lambda}{3 \pi \hat{a}}\left[\arccos \left(\frac{1}{m}\right) \sqrt{m^{2}-1}-m+1\right]+\left(m^{2}-2\right) \arccos \left(\frac{1}{m}\right)+\sqrt{m^{2}-1}+ \\
& +\left\{\frac{16 \Lambda}{3 \pi}\left[\frac{m}{\sqrt{m^{2}-1}} \arccos \left(\frac{1}{m}\right)+\frac{1}{m}-1\right]+\hat{a}\left[\frac{\sqrt{m^{2}-1}}{m}+m \arccos \left(\frac{1}{m}\right)\right]\right\} \frac{\mathrm{d} m}{\mathrm{~d} \hat{a}} .
\end{aligned}
$$

Mit der Bedingung für den kritischen Zustand und der Lösung des Kontaktproblems ergibt sich dann ein geschlossenes Gleichungssystem zur Bestimmung der kritischen Größen. An dieser Stelle soll nur auf ein Detail eingegangen werden, dass zur späteren Behandlung des Stoßproblems von Bedeutung ist. Wie man später sehen wird, endet der Stoß, wenn der Kontakt unter weggesteuerten Bedingungen seine Stabilität verliert. Aus der Abb.3.6 ist dabei klar, dass dies nur für ausreichend große Werte des Tabor-Parameters bei Konfigurationen mit direktem Kontakt passiert. Der Grenzfall, bei dem die Auflösung des Kontaktes gerade am Ende des direkten Kontaktes erfolgt, ergibt sich aus der Lösung der Gleichung

$$
\frac{3 \pi^{3}}{2^{9} \Lambda^{3}}=1-\frac{1}{\pi} \Rightarrow \Lambda \approx 0,64 .
$$

Für größere Werte von $\Lambda$ verliert der Kontakt seine Stabilität in einer Konfiguration mit direktem Kontakt. Die Konfigurationen ohne direkten Kontakt verlieren ihre Stabilität, wie einfach zu zeigen ist, unter weggesteuerten Bedingungen bei

$$
\hat{b}_{c}^{\mathrm{WS}}=\frac{32}{3 \pi^{2}} \Lambda \text {. }
$$

Abb.3.6 Normierte

Normalkraft (Zugbereich) als

Funktion der normierten

Eindrucktiefe für den

Maugis-adhäsiven

Normalkontakt von Kugeln für

verschiedene Werte des

Tabor-Parameters $\Lambda$ mit dem

JKR- und DMT-Grenzfall

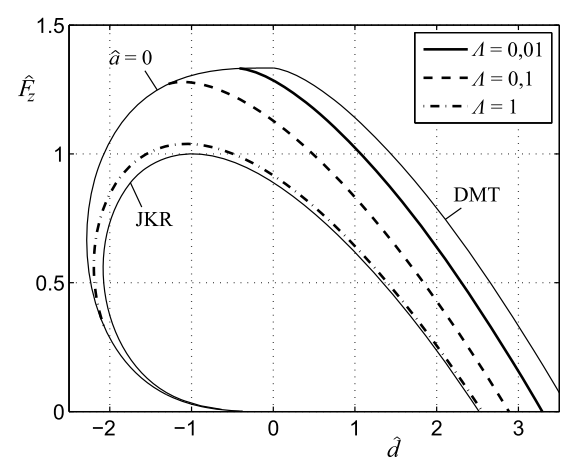




\subsubsection{Einfluss des Reibregimes}

Ebenso wie im vorherigen Unterkapitel zum Normalkontakt ohne Adhäsion wurden alle obigen Ergebnisse zum Normalkontakt mit Adhäsion unter der Voraussetzung abgeleitet, dass die Kontaktpartner elastisch ähnlich sind (und entsprechend gar keine relativen radialen Verschiebungen auftreten) oder der Kontakt reibungsfrei ist (und damit eventuell auftretende relative radiale Verschiebungen zu keinen zusätzlichen Spannungen führen). Während die Behandlung des reibungsbehafteten Normalkontaktes elastisch verschiedener Körper ohne Adhäsion zumindest physikalisch-konzeptuell keine größeren Schwierigkeiten bereitet (obwohl die rigorose mathematische Behandlung trotzdem kompliziert ist), ist das Wechselspiel von Reibung und Adhäsion bereits in den physikalischen Mechanismen noch zu großen Teilen unverstanden und Gegenstand aktueller Forschung. Dies liegt einerseits daran, dass beide Phänomene auf der kleinsten Skala sicher eng miteinander verbunden sind, diese Verbindung aber andererseits auf mesoskopischer Skala durch die Rauigkeit realer Oberflächen stark beeinflusst wird. Erst durch die Atom-Kraft-Mikroskopie (AFM) und Oberflächen-Kraft-Mikroskopie (SFM) sind seit wenigen Jahrzehnten die Kräfte auf kleinster Skala einer ausreichend genauen Messung zugänglich, die eine systematische Untersuchung des Wechselspiels von Reibung und Adhäsion ermöglicht.

Ein Beispiel für die Schwierigkeiten der theoretischen Modellierung von Adhäsion mit Reibung ist die klassische JKR-Theorie selbst: da diese auf dem reibungsfreien Normalkontakt ohne Adhäsion aufbaut, ist Reibungsfreiheit streng genommen eine ihrer Voraussetzungen; der JKR-Kontakt beschreibt also zwei Oberflächen, die mit einer unendlich großen adhäsiven Spannung aneinandergedrückt werden und trotzdem reibungsfrei aneinander abgleiten können. Trotz dieses offensichtlichen konzeptuellen Problems ist die JKRTheorie aber experimentell so oft bestätigt worden, dass ihr Nutzen und ihre Anwendbarkeit nicht zur Diskussion stehen können. Dies legt nahe, dass auch für den adhäsiven Normalkontakt (wie im nicht-adhäsiven Fall) der tatsächliche Einfluss des Reibregimes auf die Zusammenhänge zwischen den makroskopischen Kontaktgrößen in der Regel klein ist.

Ansätze zur theoretischen Modellierung der Wechselwirkung von Reibung und Adhäsion betreffen meistens den adhäsiven Tangentialkontakt; die entsprechenden Konzepte sind aber auch teilweise auf den adhäsiven Normalkontakt mit Reibung übertragbar und sollen daher an dieser Stelle kurz geschildert werden, ohne genauer auf die im folgenden Unterkapitel ausgeführten Eigenschaften des Tangentialkontaktes einzugehen.

Die erste Untersuchung zu dem Thema stammt von Savkoor und Briggs [44]. Sie studierten den JKR-adhäsiven Kontakt ohne Gleiten unter einer tangentialen Belastung und stellten durch eine Energie-Bilanz fest, dass die Tangentialbelastung zu einer Reduktion der Adhäsion führt. Diese Reduktion war im Experiment allerdings geringer als durch die Theorie vorausgesagt. Da die tangentiale Verschiebung eines kreisförmigen Kontaktgebiets ohne Gleiten zu der gleichen Form der Spannungskonzentration führt wie die Indentierung durch einen flachen zylindrischen Stempel (und damit wie im reibungsfreien JKR-Kontakt), ist dieser Ansatz äquivalent zur Betrachtung der Spannungskonzentration am Rand des 
adhäsiven Kontaktes mit mehreren „Rissmoden“: während die reine Normalbelastung einem Mode-I-Riss entspricht, kommt es durch die Tangentialbelastung zu Mode-II- und ModeIII-Komponenten. Durch eine Zusammenfassung der entsprechenden Spannungskonzentrationsfaktoren können mit dem Griffith-Kriterium der Linear-Elastischen Bruchmechanik adhäsive, tangential belastete Kontakte behandelt werden [45, 46]. Dies setzt allerdings voraus, dass alle Moden gleichwertig zur Auflösung des Kontaktes beitragen, was nicht unbedingt der Fall sein muss [47].

Mit dem Dugdale-Maugis-Modell der Adhäsion konnte man schließlich auch den Fall von partiellem oder vollständigem Gleiten im Kontakt betrachten [46, 48]. Hier kommt als weiterer Effekt die Dissipation von mechanischer Energie durch das Gleiten ins Spiel, d.h. nicht die ganze bei der Auflösung des Kontaktes freiwerdende elastische Energie kann in Oberflächentrennungsarbeit umgesetzt werden. Auch die Reversibilität der adhäsiven Kontaktbildung und -auflösung ist in diesem Fall nicht garantiert [47, 49]. Waters und Guduru [49] untersuchten daher eine durch die Anwesenheit mehrerer Moden erhöhte effektive Oberflächenenergie; Experimente im Bereich sehr geringen lokalen Gleitens stützten ihre Theorie. Während Johnson [46] feststellte, dass bei vollständigem Gleiten die tangentiale Kraft die Wirkung der Adhäsion reduziert, machten Kim et al. [48] darauf aufmerksam, dass auf mikroskopischer Skala die Scherspannung in einem einzelnen gleitenden Mikrokontakt näherungsweise konstant und gleich der Scherfestigkeit ist. Bei konstanter Scherspannung in einem gleitenden Kontakt kann die tangentiale Belastung aber sogar zu einer Erhöhung der Adhäsion führen [50].

Ein minimales Modell mit Amontons-Coulomb-Reibung und Dugdale-Maugis-Adhäsion benutzten Popov und Dimaki [51] für den adhäsiven Tangentialkontakt. Sie stellen fest, dass (zumindest bei kleinen Gebieten lokalen Gleitens) die Adhäsion zu einer zusätzlichen Anpresskraft der Oberflächen führt, die die Reibkraft überwinden muss. Filippov et al. [52] untersuchten ein nanoskopisches Modell von gebildeten und aufgelösten Kontakten (linearen Federn) unter tangentialer Belastung.

Der adhäsive Normalkontakt eines Kegels ohne Gleiten im JKR-Grenzfall wurde von Borodich et al. [53] gelöst, allerdings berücksichtigten die Autor*innen dabei nicht den Beitrag radialer Riss-Moden zur Auflösung des Kontaktes; stattdessen verwendeten sie die entsprechende Lösung des nicht-adhäsiven Problems ohne Gleiten und die JKR-Theorie in der ursprünglichen Formulierung für reibungsfreie Kontakte, was wiederum physikalisch nicht ganz einleuchtet. In der gleichen Näherung wurde von Lyashenko et al. [54] der JKR-adhäsive ebene Stoß ohne Gleiten einer starren Kugel auf einen elastischen Halbraum untersucht.

Mergel et al. [55] schlugen mehrere kontinuumsmechanische Modelle für Kontakte mit Reibung und Adhäsion auf der Basis von Amontons-Coulomb-Reibung und dem LennardJones-Potential vor. Die Autor*innen gaben außerdem eine ausführliche Literaturübersicht über bestehende Modellansätze zu adhäsiver Reibung, insbesondere in bio-adhäsiven Systemen. 


\subsection{Tangentialkontakt}

Das folgende Unterkapitel ist axialsymmetrischen Kontakten gewidmet, die sowohl in normaler Richtung $z$ als auch unidirektional in tangentialer Richtung $x$ belastet sind. Dabei sei grundsätzlich angenommen, dass beide Kontaktpartner elastisch ähnlich sind und damit die Kontaktaufgaben in normaler und tangentialer Richtung elastisch entkoppeln. Die analytische Behandlung elastisch gekoppelter Probleme mit Reibung ist äußerst kompliziert und würde den Rahmen dieses Buches sprengen. Es sei in diesem Zusammenhang aber auf die ausgezeichnete Monografie von Barber [13, S. 184 ff.] verwiesen.

Unter der Voraussetzung elastischer Ähnlichkeit kann man zunächst das Tangentialkontaktproblem unter Annahme der Abwesenheit lokalen Gleitens lösen. Die resultierenden Schubspannungen zeigen am Rand des Kontaktes das gleiche Singularitätsverhalten wie die Druckverteilung bei der Indentierung durch einen flachen zylindrischen Stempel. Da die Normalspannungen am Rand von (nicht-adhäsiven) Kontakten konvexer Oberflächen verschwinden, breitet sich daher durch die tangentiale Belastung vom Rand des Kontaktes ein ringförmiges Gleitgebiet ins Innere des Kontaktes aus. Wenn das innere Haftgebiet vollständig verschwindet, beginnt der Kontakt global zu gleiten.

\subsubsection{Tangentialkontakt ohne Gleiten}

Zunächst betrachte man das Tangentialkontaktproblem ohne Gleiten zwischen einem starren Indenter und einem elastischen Halbraum. Es ist klar, dass die tangentialen Verschiebungen im Kontakt ohne Gleiten einer Starrkörperverschiebung des Kontaktgebiets um $u_{x, 0}$ entsprechen müssen. Wie sich mithilfe der Fundamentallösung aus Gl.(3.1) ohne größere Schwierigkeiten zeigen lässt, siehe z. B. Johnson [11, S. 71 ff.], kann dieses Problem durch die Spannungsverteilung

$$
\sigma_{x z}(r)=\frac{\tilde{G}}{\pi} \frac{u_{x, 0}}{\sqrt{a^{2}-r^{2}}}, \quad r \leq a,
$$

mit dem in Gl.(3.15) eingeführten effektiven Schubmodul $\tilde{G}$, gelöst werden. Diese Schubspannungsverteilung hat offensichtlich die gleiche Form wie die Druckverteilung unter einem flachen zylindrischen Stempel in Gl. (3.22) und daher das gleiche Singularitätsverhalten am Rand des Kontaktes. Die gesamte Tangentialkraft beträgt

$$
F_{x}=2 \tilde{G} a u_{x, 0},
$$

womit die tangentiale Steifigkeit des vollständig haftenden Kontaktes zu

$$
k_{x}:=\frac{\mathrm{d} F_{x}}{\mathrm{~d} u_{x, 0}}=2 \tilde{G} a
$$

bestimmt werden kann. 


\subsubsection{Cattaneo-Mindlin-Theorie}

Die Grundlage der Untersuchung des parabolischen Tangentialkontaktes unter Berücksichtigung des lokalen Gleitens sind die Verschiebungen der Oberfläche eines elastischen Halbraums unter der Einwirkung der rotationssymmetrischen Schubspannungsverteilung

$$
\sigma_{x z}(r)=\frac{\sigma_{1}}{a} \sqrt{a^{2}-r^{2}}, \quad r \leq a,
$$

die analog zur Hertzschen Druckverteilung (3.37) ist. Mithilfe der Fundamentallösung (3.1) erhält man für die Verschiebungen der Halbraumoberfläche innerhalb des Kreises $r \leq a$

$$
\begin{aligned}
& u_{x}(x, y)=\frac{\pi \sigma_{1}}{32 G a}\left[4(2-v) a^{2}-(4-3 v) x^{2}-(4-v) y^{2}\right], \quad r \leq a, \\
& u_{y}(x, y)=\frac{\pi \sigma_{1} v x y}{16 G a}, \quad r \leq a,
\end{aligned}
$$

und außerhalb des Kontaktkreises

$$
\begin{aligned}
u_{x}(x, y) & =\frac{\sigma_{1}}{16 G a}(4-2 v)\left[a \sqrt{r^{2}-a^{2}}+\left(2 a^{2}-r^{2}\right) \arcsin \left(\frac{a}{r}\right)\right]+ \\
& +\frac{\sigma_{1}}{16 G a} v\left(x^{2}-y^{2}\right)\left[\arcsin \left(\frac{a}{r}\right)+\frac{a}{r}\left(\frac{2 a^{2}}{r^{2}}-1\right) \sqrt{1-\frac{a^{2}}{r^{2}}}\right], \quad r>a \\
u_{y}(x, y) & =\frac{\sigma_{1} v x y}{8 G a}\left[\arcsin \left(\frac{a}{r}\right)+\frac{a}{r}\left(\frac{2 a^{2}}{r^{2}}-1\right) \sqrt{1-\frac{a^{2}}{r^{2}}}\right], \quad r>a
\end{aligned}
$$

Die ausführliche Herleitung der aufgeführten Gleichungen ist im Anhang dargelegt. Es sei außerdem angemerkt, dass die von Johnson [11, S. 74 f.] angegebenen Ergebnisse im Fall der Verschiebungen außerhalb des Kontaktkreises leicht fehlerhaft sind.

Man betrachte nun folgende einfache Belastungsgeschichte: eine starre Kugel wird mit einer konstanten Normalkraft in den elastischen Halbraum gedrückt und anschließend tangential verschoben. Dann ist klar, dass bei einem unendlichen Reibbeiwert $\mu$ die Oberflächen im Kontakt vollständig aneinander haften und die Schubspannungsverteilung im Kontakt daher durch Gl. (3.91) gegeben ist. Diese Spannungen divergieren aber am Rand des Kontaktes, d.h. der Kontakt kann bei einem endlichen Reibbeiwert nicht vollständig haften, da die Spannungen am Rand des Kontaktgebiets grundsätzlich die Haftbedingung verletzen. Der Kontakt setzt sich also aus einem inneren Haft- und einem Gleitgebiet zusammen.

Cattaneo [56] und Mindlin [57] stellten unabhängig voneinander fest, dass aus den Verschiebungen (3.95) folgt, dass eine passende Differenz zweier Spannnungsverteilungen in der Form (3.94) mit zwei verschiedenen Radien $a$ und $c<a$, 


$$
\begin{array}{ll}
\sigma_{x z}^{I}(r)=\frac{\sigma_{1}}{a} \sqrt{a^{2}-r^{2}}, & r \leq a, \\
\sigma_{x z}^{I I}(r)=\frac{\sigma_{2}}{c} \sqrt{c^{2}-r^{2}}, & r \leq c,
\end{array}
$$

eine konstante Verschiebung $u_{x}$ innerhalb des Gebiets $r \leq c$ erzeugen kann, falls

$$
\frac{\sigma_{1}}{a}=\frac{\sigma_{2}}{c}
$$

Da die tangentiale Verschiebung innerhalb des Gebiets $r \leq c$ konstant ist, hat $c$ dann die Bedeutung des Radius des Haftgebiets. Im Gleitgebiet ist

$$
\left|\sigma_{x z}\right|=\mu\left|\sigma_{z z}\right|, \quad c<r \leq a,
$$

und damit wegen Gl. (3.37)

$$
\sigma_{1}=\frac{2 \mu \tilde{E} a}{\pi \tilde{R}} \operatorname{sgn}\left(u_{x, 0}\right) .
$$

Setzt man voraus, dass es im Kontakt nur Normalspannungen und uni-direktionale Schubspannungen $\sigma_{x z}$ gibt, und dass die aus diesen Schubspannungen resultierenden Verschiebungen $u_{y}$ vernachlässigt werden können (diese beiden Annahmen werden häufig zur „CattaneoMindlin-Näherung“ zusammengefasst), ist das Kontaktproblem damit gelöst. Die Schubspannungen im Haftgebiet sind

$$
\sigma_{x z}(r)=\frac{2 \mu \tilde{E}}{\pi \tilde{R}}\left(\sqrt{a^{2}-r^{2}}-\sqrt{c^{2}-r^{2}}\right) \operatorname{sgn}\left(u_{x, 0}\right) .
$$

Die gesamte Schubspannungsverteilung ist in normierter Darstellung für verschiedene Haftradien in Abb. 3.7 gezeigt.

Abb. 3.7 Verteilung der normierten Schubspannungen ( $p_{0}$ bezeichnet den mittleren Druck) bei verschiedenen Haftradien für das Cattaneo-Mindlin-Problem. Die dünne Linie bezeichnet die Verteilung bei vollständigem Gleiten. Diese entspricht bis auf den Faktor $\mu$ der Druckverteilung

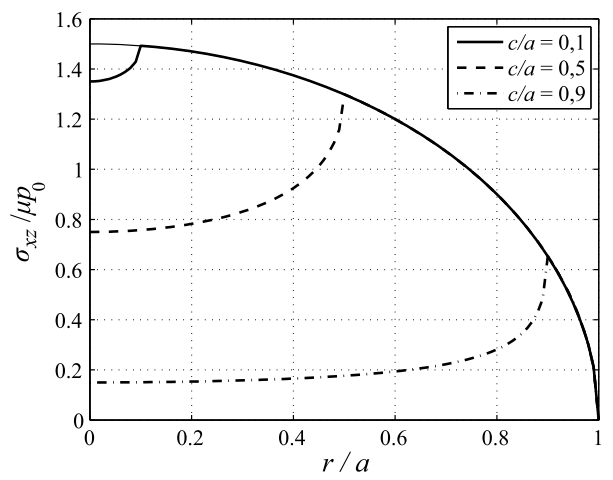


Die Vernachlässigung der Verschiebungen $u_{y}$ ist dabei problematisch. Tatsächlich gibt es, wie aus den Gl. (3.96) und (3.98) klar hervorgeht, bei der angenommenen Verteilung der Tangentialspannungen relative Verschiebungen $\Delta u_{y}$ im Gleitgebiet. Die Schubspannungen im Gleitgebiet sind daher nicht den relativen Verschiebungen entgegengerichtet, was die Isotropie des Reibgesetzes verletzt. Johnson [11, S. 219] gab an, dass das Verhältnis der relativen Verschiebungen $\Delta u_{y} / \Delta u_{x}$ von der Größenordnung $v /(4-2 v)$ ist (die Richtungsabweichung beträgt damit wenige Grad) und schlussfolgerte, dass die Cattaneo-MindlinLösung eine gute Näherung der exakten Lösung darstellt. Dies bestätigten Munisamy et al. [58] durch Vergleiche mit der widerspruchsfreien (numerischen) Lösung des Problems.

\subsubsection{Erweiterung auf beliebige Belastungsgeschichten}

Bisher wurde nur die einfachste Belastungsgeschichte eines Tangentialkontaktes betrachtet (konstante Normalkraft, monoton wachsende Tangentialkraft). Im Gegensatz zum reinen Normalkontaktproblem ist aber die Lösung des Tangentialkontaktproblems nicht nur von der momentanen Konfiguration des Kontaktes bestimmt, sondern von der gesamten bisherigen Belastungsgeschichte, da im Haftgebiet Teile der Belastungsgeschichte in Form von Tangentialspannungen gespeichert werden. Der Kontakt besitzt in diesem Sinne ein „Gedächtnis“. Durch die Energie-Dissipation im Gleitgebiet kommt es außerdem bei zyklischer Belastung zur Hysterese. Die Angabe eines expliziten Gesetzes für die Tangentialkraft $F_{x}$ ist daher nur mit Kenntnis der Belastungsgeschichte möglich.

Mindlin und Deresiewicz [59] gaben einen geschlossenen Regelsatz an, mit dem man das Kontaktproblem bei einer beliebigen Belastungsgeschichte lösen kann, und untersuchten eine Vielzahl unterschiedlicher Belastungsfälle. Eine elegantere Formulierung der Lösung für beliebige Belastungsgeschichten stammt von Jäger [60]. Beide genannten Publikationen sind natürlich unter den Annahmen der Cattaneo-Mindlin-Näherung zustande gekommen.

Die Grundidee der Jägerschen Lösung ist, dass man die Tangentialspannungen bei einer beliebigen Belastungsgeschichte als eine Superposition von Cattaneo-Mindlin-Spannungen

$$
\sigma_{B}\left(r ; a_{i}, a_{j}\right):=\frac{2 \mu \tilde{E}}{\pi \tilde{R}} \begin{cases}\sqrt{a_{i}^{2}-r^{2}}-\sqrt{a_{j}^{2}-r^{2}}, & r \leq a_{j}, \\ \sqrt{a_{i}^{2}-r^{2}}, & a_{j}<r \leq a_{i},\end{cases}
$$

konstruieren kann. Nach einem von Jäger [61] und Ciavarella [62, 63] unabhängig voneinander gefundenen Theorem lässt sich Gl. (3.105) für alle axialsymmetrischen Kontakte im Rahmen der Cattano-Mindlin-Näherung zu

$$
\sigma_{B}\left(r ; a_{i}, a_{j}\right)=-\mu \begin{cases}\sigma_{z z}\left(r ; a_{i}\right)-\sigma_{z z}\left(r ; a_{j}\right), & r \leq a_{j}, \\ \sigma_{z z}\left(r ; a_{i}\right) \quad, & a_{j}<r \leq a_{i},\end{cases}
$$


verallgemeinern. Die zugehörigen Werte der tangentialen Starrkörperverschiebung und Tangentialkraft können als

$$
\begin{aligned}
u_{x, 0, B}\left(a_{i}, a_{j}\right) & :=\frac{\mu \tilde{E}}{\tilde{G}}\left(\frac{a_{i}^{2}}{\tilde{R}}-\frac{a_{j}^{2}}{\tilde{R}}\right)=\frac{\mu \tilde{E}}{\tilde{G}}\left[g\left(a_{i}\right)-g\left(a_{j}\right)\right], \\
F_{x, B}\left(a_{i}, a_{j}\right) & :=\frac{4}{3} \frac{\mu \tilde{E}}{\tilde{R}}\left(a_{i}^{3}-a_{j}^{3}\right)=-\mu\left[F_{z}\left(a_{i}\right)-F_{z}\left(a_{j}\right)\right]
\end{aligned}
$$

geschrieben werden. Man überzeugt sich außerdem leicht davon, dass die eingeführten Basisspannungen die Superpositionsregel

$$
\sigma_{B}\left(r ; a_{i}, a_{j}\right)+\sigma_{B}\left(r ; a_{j}, a_{k}\right)=\sigma_{B}\left(r ; a_{i}, a_{k}\right), \quad a_{i}>a_{j}>a_{k},
$$

erfüllen.

Man betrachte nun einen Kontakt nach einer einzigen Cattaneo-Mindlin-Belastung. Das Kontaktgebiet mit dem Radius $a_{1}$ besteht aus einem Haftgebiet mit dem Radius $c_{1}$ und einem Gleitgebiet $c_{1}<r \leq a_{1}$. Ohne Beschränkung der Allgemeinheit sei angenommen, dass $u_{x, 0,1}>0$. Die Verteilung der Tangentialspannungen ist also durch

$$
\sigma_{x z}(r)=\sigma_{B}\left(r ; a_{1}, c_{1}\right)
$$

gegeben. Nun wird die Eindrucktiefe um $\Delta d$ und anschließend die tangentiale Starrkörperverschiebung um $\Delta u_{x, 0}$ verändert ${ }^{7}$. Da sich bei jeder (noch so kleinen) tangentialen Belastung vom Rand des Kontaktes ein Gleitgebiet ausbreitet, zerfällt das neue Kontaktgebiet mit dem Radius $a_{2}$ wiederum in ein Haftgebiet mit dem Radius $c_{2}$ und ein Gleitgebiet $c_{2}<r \leq a_{2}$. Grundsätzlich müssen zwei Fälle unterschieden werden: ein wachsendes Kontaktgebiet mit $a_{2}>a_{1}$ (also $\Delta d>0$ ) und ein schrumpfendes Kontaktgebiet mit $a_{2}<a_{1}$ (also $\Delta d<0$ ). Der Spezialfall eines konstanten Kontaktradius (also $\Delta d=0$ ) ergibt sich in beiden Varianten elementar als Grenzfall.

\section{Kontaktgebiet wächst}

Wenn das Kontaktgebiet wächst, gibt es zunächst - bevor die zusätzliche tangentiale Verschiebung $\Delta u_{x, 0}$ aufgebracht wird - einen Punkt vollständigen Haftens. Danach breitet sich das Gleitgebiet erneut vom Rand des Kontaktes nach innen aus. Solange $c_{2}>a_{1}$, lauten die Randbedingungen für das Differenzproblem (zwischen den Zuständen mit den Indizes „,1“ und ,2") dann wie folgt:

$$
\begin{aligned}
\Delta u_{x}(r) & =\Delta u_{x, 0}, \quad r \leq c_{2}, \\
\left|\Delta \sigma_{x z}(r)\right| & =\mu\left|\sigma_{z z}(r)\right|, \quad c_{2}<r \leq a_{2} .
\end{aligned}
$$

Dies ist aber selbst ein Cattaneo-Mindlin-Problem mit der entsprechenden Lösung

\footnotetext{
${ }^{7}$ Beide Inkremente können aber müssen nicht zwangsläufig infinitesimal sein [60].
} 


$$
\Delta \sigma_{x z}(r)=\operatorname{sgn}\left(\Delta u_{x, 0}\right) \sigma_{B}\left(r ; a_{2}, c_{2}\right)
$$

Es wird also eine weitere Cattaneo-Mindlin-Spannung zu der bereits bestehenden linear superponiert. Die tangentiale Starrkörperverschiebung verändert sich um

$$
\left|\Delta u_{x, 0}\right|=u_{x, 0, B}\left(a_{2}, c_{2}\right)
$$

das bedeutet, die Bedingung $c_{2}>a_{1}$ führt auf die Forderung

$$
\left|\Delta u_{x, 0}\right|<\mu \frac{\tilde{E}}{\tilde{G}}\left[g\left(a_{2}\right)-g\left(a_{1}\right)\right]=\mu \frac{\tilde{E}}{\tilde{G}} \Delta d .
$$

Falls $c_{1}<c_{2}<a_{1}$, muss man zunächst die Schubspannungen im Ring $c_{2}<r \leq a_{1}$ durch eine Verteilung

$$
\Delta \sigma_{x z, I}(r)=-\sigma_{B}\left(r ; a_{1}, c_{2}\right)
$$

löschen. Anschließend wird wiederum eine weitere Verteilung

$$
\Delta \sigma_{x z, I I}(r)=\operatorname{sgn}\left(\Delta u_{x, 0}\right) \sigma_{B}\left(r ; a_{2}, c_{2}\right)
$$

linear superponiert. Im Fall $\Delta u_{x, 0}>0$ ist die gesamte neue Spannungsverteilung wegen der Superpositionsregel (3.109) durch

$$
\sigma_{x z}(r)=\sigma_{B}\left(r ; a_{1}, c_{1}\right)-\sigma_{B}\left(r ; a_{1}, c_{2}\right)+\sigma_{B}\left(r ; a_{2}, c_{2}\right)=\sigma_{B}\left(r ; a_{2}, c_{1}\right),
$$

gegeben, das Haftgebiet schrumpft also sofort auf den Radius $c_{1}$. Der Fall $c_{1}<c_{2} \leq a_{1}$ existiert daher nur, falls $\Delta u_{x, 0}<0$, d. h. falls ein Umkehrpunkt der Tangentialbewegung auftritt. Da die gesamte Differenz der tangentialen Starrkörperverschiebung unter diesen Umständen als

$$
\Delta u_{x, 0}=-\left[u_{x, 0, B}\left(a_{1}, c_{2}\right)+u_{x, 0, B}\left(a_{2}, c_{2}\right)\right]
$$

geschrieben werden kann, führt die Bedingung $c_{2}>c_{1}$ auf die Forderung

$$
\Delta u_{x, 0}>-2 u_{x, 0,1}-\mu \frac{\tilde{E}}{\tilde{G}} \Delta d .
$$

Falls $c_{2} \leq c_{1}$, wird die gesamte ursprünglich vorhandene Spannungsverteilung gelöscht und neu überschrieben. Man erhält

$$
\sigma_{x z}(r)=\operatorname{sgn}\left(\Delta u_{x, 0}\right) \sigma_{B}\left(r ; a_{2}, c_{2}\right)
$$

und entsprechend

$$
u_{x, 0,2}=\operatorname{sgn}\left(\Delta u_{x, 0}\right) u_{x, 0, B}\left(a_{2}, c_{2}\right) .
$$




\section{Kontaktgebiet schrumpft}

Wenn das Kontaktgebiet schrumpft, d.h. $\Delta d<0$, muss zunächst der Ring $a_{2}<r \leq a_{1}$ von Tangentialspannungen befreit werden, während das neue Kontaktgebiet $r \leq a_{2}$ haftet. Dazu ist eine Spannungsverteilung

$$
\Delta \sigma_{x z, I}(r)=-\sigma_{B}\left(r ; a_{1}, a_{2}\right)
$$

und eine Verschiebung

$$
\Delta u_{x, 0, I}=-u_{x, 0, B}\left(a_{1}, a_{2}\right)=\mu \frac{\tilde{E}}{\tilde{G}} \Delta d
$$

notwendig. Dann verkleinert man das Kontaktgebiet. Anschließend kann analog wie im vorher betrachteten Fall verfahren werden (wobei das Kontaktgebiet sogar konstant bleibt).

\section{Zusammenfassung der verschiedenen Fälle}

Die resultierenden Spannungen nach der Belastung kann man für alle möglichen Fälle zu

$\sigma_{x z}(r)=\left\{\begin{array}{l}\sigma_{B}\left(r ; a_{1}, c_{1}\right)+\operatorname{sgn}\left(\Delta u_{x, 0}\right) \sigma_{B}\left(r ; a_{2}, c_{2}\right), \quad \Delta d \geq 0 \wedge\left|\Delta u_{x, 0}\right| \leq \mu \frac{\tilde{E}}{\tilde{G}} \Delta d, \\ \sigma_{B}\left(r ; c_{2}, c_{1}\right)-\sigma_{B}\left(r ; a_{2}, c_{2}\right), \quad-\mu \frac{\tilde{E}}{\tilde{G}}|\Delta d| \geq \Delta u_{x, 0}>-2 u_{x, 0,1}-\mu \frac{\tilde{E}}{\tilde{G}} \Delta d, \\ \operatorname{sgn}\left(\Delta u_{x, 0}-\mu \frac{\tilde{E}}{\tilde{G}} \Delta d\right) \sigma_{B}\left(r ; a_{2}, c_{2}\right), \quad \text { sonst, }\end{array}\right.$

zusammenfassen. Der Radius $c_{2}$ des neuen Haftgebiets ergibt sich dabei, je nach den drei in Gl. (3.125) unterschiedenen Möglichkeiten, nach den Gl. (3.114), (3.119) oder (3.122). Die gesamte Tangentialkraft ergibt sich durch die in Gl. (3.125) beschriebene Superposition von Cattaneo-Mindlin-Kräften $F_{x, B}$ nach Gl. (3.108).

\section{Allgemeine Ausgangssituation}

Die obigen Beziehungen können ohne Schwierigkeiten in iterativer Form für eine beliebige Ausgangssituation verallgemeinert werden. Es sei $\sigma_{x z, n}$ die Spannungsverteilung nach $n$ Verschiebungsinkrementen $\Delta d_{i}$ und $\Delta u_{x, 0, i}$. Dann ist

$$
\sigma_{x z, n}(r)=\left\{\begin{array}{l}
\sigma_{x z, n-1}(r)+\operatorname{sgn}\left(\Delta u_{x, 0, n}\right) \sigma_{B}\left(r ; a_{n}, c_{n}\right), 1 \\
\sigma_{x z, n-2}(r)+\operatorname{sgn}\left(\Delta u_{x, 0, n-1}\right) \sigma_{B}\left(r ; c_{n}, c_{n-1}\right)-\operatorname{sgn}\left(\Delta u_{x, 0, n}\right) \sigma_{B}\left(r ; a_{n}, c_{n}\right), \\
\sigma_{x z, n-2}(r)+\operatorname{sgn}\left(\Delta u_{x, 0, n}-\mu \frac{\tilde{E}}{\tilde{G}} \Delta d_{n}\right) \sigma_{B}\left(r ; a_{n}, c_{n}\right), \text { sonst }
\end{array}\right.
$$

wobei die Bedingungen durch

$$
\text { (1): } \Delta d_{n} \geq 0 \wedge\left|\Delta u_{x, 0, n}\right| \leq \mu \frac{\tilde{E}}{\tilde{G}} \Delta d_{n},
$$




$$
\text { (2): }-\mu \frac{\tilde{E}}{\tilde{G}}\left|\Delta d_{n}\right| \geq \Delta u_{x, 0, n}>-\mu \frac{\tilde{E}}{\tilde{G}}\left[\Delta d_{n}+2 d_{n-1}-2 g\left(c_{n-1}\right)\right]
$$

gegeben sind. Wenn der sonstige Fall in Gl. (3.126) eintritt, wurde offenbar das Inkrement mit dem Index $n-1$ vollständig gelöscht. Man muss dann den Index um Eins reduzieren.

Die ganze Lösung lässt sich als Algorithmus formulieren, was Aleshin et al. [64, 65] benutzten, um „Gedächtnis-Diagramme“ (memory diagrams) von Tangentialkontakten mit beliebigen Belastungsgeschichten in der Cattaneo-Mindlin-Näherung zu erstellen. Eine andere Deutung der gezeigten Kontaktlösungen liefert außerdem die im nächsten Kapitel dieses Buches erläuterte Methode der Dimensionsreduktion.

\subsection{Torsionskontakt}

In diesem Unterkapitel stehen axialsymmetrische Kontakte im Mittelpunkt, die sowohl durch eine Normalkraft als auch durch ein Torsionsmoment um die Normalenachse belastet werden. Torsionskontakte besitzen ähnliche Eigenschaften wie die im vorigen Unterkapitel behandelten Tangentialkontakte: das Kontaktgebiet zerfällt in der Regel in ein Haft- und ein Gleitgebiet und der Kontakt weist daher Gedächtnis- und Hysterese-Effekte auf. Der Aufbau dieses Unterkapitels ist deswegen weitgehend analog zu dem vorherigen: zuerst wird das Kontaktproblem ohne Gleiten gelöst und anschließend lokales Gleiten durch die bei vollständigem Haften am Rand des Kontaktes divergierenden Schubspannungen berücksichtigt.

\subsubsection{Torsionskontakt ohne Gleiten}

Es soll zunächst das statische, rotationssymmetrische Reissner-Sagoci-Problem für einen elastischen Halbraum gelöst werden. Die gemischten Randbedingungen für die Schubspannung und die torsionale Verschiebung des Halbraums sind

$$
\begin{aligned}
\sigma_{\varphi z}(r) & =0, \quad r>a, \\
u_{\varphi}(r) & =r[\varphi-\gamma(r)], \quad r \leq a,
\end{aligned}
$$

d.h. es wird innerhalb eines kreisförmigen Kontaktgebiets ohne Gleiten eine vorgegebene torsionale Verschiebung in den elastischen Halbraum eingeprägt. Es ist $\gamma(r)$ eine beliebige Funktion mit $\gamma(0)=0$ und $\varphi$ ein Winkel um sicherzustellen, dass dies keine Beschränkung der Allgemeinheit darstellt. Physikalisch beschreibt $\gamma(r)$ die Abweichung der torsionalen Verschiebungen von einer reinen Starrkörperrotation um $\varphi$. Das einzige tatsächliche Kontaktproblem ohne Gleiten, das durch diese Randbedingungen beschrieben wird, ist der Kontakt mit einem starren flachen zylindrischen Stempel, für den offenbar $\gamma(r) \equiv 0$. Trotzdem benötigt man für die Behandlung des Kontaktproblems mit lokalem Gleiten den allgemeinen Fall für ein beliebiges $\gamma(r)$, da für Torsionskontakte kein dem Ciavarella-Jäger-Theorem 
für Tangentialkontakte analoges Prinzip existiert, mit dem das Kontaktproblem auf den reibungsfreien Normalkontakt zurückgeführt werden könnte.

Das Verschiebungsfeld in Gl. (3.130) kann in Analogie zur allgemeinen Lösung des rotationssymmetrischen reibungs- und adhäsionsfreien Normalkontaktproblems als Integral von infinitesimalen Starrkörperrotationen mit wachsendem Kontaktradius $\tilde{a}$ aufgefasst werden [10]. Man benötigt also wiederum zuallererst die Lösung für die Torsion durch einen flachen zylindrischen Stempel, die z. B. bei Johnson [11, S. 81 f.] dokumentiert ist. Der Zusammenhang zwischen dem Torsionsmoment $M_{z}$ und dem Verdrehwinkel $\varphi$ ist durch

$$
M_{z}=\frac{16 G}{3} \varphi a^{3}
$$

gegeben. Die Spannungsverteilung im Kontakt ist

$$
\sigma_{\varphi z}(r)=\frac{4 G r \varphi}{\pi \sqrt{a^{2}-r^{2}}}, \quad r \leq a,
$$

und die Verschiebungen außerhalb des Kontaktgebiets sind

$$
u_{\varphi}(r)=\frac{2 \varphi}{\pi}\left[r \arcsin \left(\frac{a}{r}\right)-a \sqrt{1-\frac{a^{2}}{r^{2}}}\right], \quad r>a .
$$

Analog zu Gl. (3.25) kann man die Funktion

$$
\varphi=\phi(a)
$$

einführen. Nun werden analog zum reibungsfreien Normalkontakt die einzelnen Starrkörperrotationen mit wachsendem Kontaktradius summiert. Da die folgenden Ergebnisse, wie oben beschrieben, nur für den Kontakt mit Gleiten kontaktmechanische Relevanz haben, sollen sie sich auf den Fall beschränken, dass die Schubspannungen am Rand des Kontaktes (wie die Normalspannungen am Rand des Kontaktes mit gekrümmten Oberflächen) verschwinden. Man erhält nach partieller Integration für das gesamte Torsionsmoment

$$
M_{z}=16 G \int_{0}^{a} x^{2}[\varphi-\phi(x)] \mathrm{d} x .
$$

Die gesamte Schubspannung im Kontaktgebiet ist

$$
\sigma_{\varphi z}(r)=\frac{4 G r}{\pi} \int_{r}^{a} \frac{\phi^{\prime}(x)}{\sqrt{x^{2}-r^{2}}} \mathrm{~d} x, \quad r \leq a,
$$

und die Verschiebungen sind 


$$
u_{\varphi}(r)=\frac{2}{\pi} \int_{0}^{\min (r, a)}\left[r \arcsin \left(\frac{x}{r}\right)-x \sqrt{1-\frac{x^{2}}{r^{2}}}\right] \phi^{\prime}(x) \mathrm{d} x+r \int_{\max (r, a)}^{a} \phi^{\prime}(x) \mathrm{d} x,
$$

was sich zu

$$
u_{\varphi}(r)=\frac{4}{\pi r} \int_{0}^{\min (r, a)} \frac{x^{2}[\varphi-\phi(x)]}{\sqrt{r^{2}-x^{2}}} \mathrm{~d} x
$$

zusammenfassen lässt. Die Funktionen $\phi(x)$ und $\gamma(r)$ lassen sich wie im Fall des reibungsfreien Normalkontaktes durch geeignete Abeltransformationen ineinander überführen. Man erhält aus dem Vergleich der Gl. (3.130) und (3.138)

$$
\gamma(r)=\frac{4}{\pi r^{2}} \int_{0}^{r} \frac{x^{2} \phi(x)}{\sqrt{r^{2}-x^{2}}} \mathrm{~d} x
$$

und durch Inversion dieser Beziehung [14, S. 353]

$$
\phi(x)=\frac{1}{2 x^{2}} \frac{\mathrm{d}}{\mathrm{d} x}\left[\int_{0}^{|x|} \frac{r^{3} \gamma(r)}{\sqrt{x^{2}-r^{2}}} \mathrm{~d} r\right]
$$

\subsubsection{Torsionskontakt mit Gleiten}

Auf Grundlage der Überlegungen aus dem vorherigen Abschnitt kann man nun den überlagerten Normal- und Torsionskontakt zwischen einem rotationssymmetrischen starren Indenter mit dem Profil $f(r)$ und einem elastischen Halbraum untersuchen, wobei im Kontakt Haftung und Reibung nach dem Amontons-Coulomb-Gesetz angenommen sei.

Der starre Indenter werde um $d$ in den Halbraum eingedrückt und anschließend um den Winkel $\varphi>0$ um seine Achse verdreht. Wie im Fall des Tangentialkontaktes in der Cattaneo-Mindlin-Näherung wird sich im Inneren des Kontaktes ein Haftgebiet $r \leq c$ mit dem Haftradius $c \leq a$ ausbilden, das von einem ringförmigen Gleitgebiet $c<r \leq a$ umgeben ist. Im Haftgebiet besteht die torsionale Verschiebung aus einer reinen Starrkörperdrehung um den Winkel $\varphi$ und im Gleitgebiet herrscht Coulumbsche Reibung. Die gemischten Randbedingungen für das Torsionsproblem lauten daher wie folgt:

$$
\begin{aligned}
u_{\varphi}(r) & =r \varphi, \quad r \leq c, \\
\sigma_{\varphi z}(r) & =-\mu \sigma_{z z}(r), \quad r>c .
\end{aligned}
$$

Aus dem Vergleich von Gl.(3.138) mit der Randbedingung (3.141) ist klar, dass 


$$
\phi(x)=0, \quad|x| \leq c,
$$

sein muss. Aus dem Vergleich von Gl. (3.136) mit der Randbedingung (3.142) erhält man die Beziehung

$$
\sigma_{z z}(r)=-\frac{4 G r}{\mu \pi} \int_{r}^{a} \frac{\phi^{\prime}(x)}{\sqrt{x^{2}-r^{2}}} \mathrm{~d} x, \quad c<r \leq a .
$$

Dies ist eine Abeltransformation, die mit dem Ergebnis [14, S. 353]

$$
\phi(x)=\frac{\mu}{2 G} \int_{x}^{a} \frac{\sigma_{z z}(r)}{\sqrt{r^{2}-x^{2}}} \mathrm{~d} r+C, \quad c<|x| \leq a,
$$

invertiert werden kann. Aus der Lösung des Normalkontaktproblems (siehe Gl.(3.28)) ist $\sigma_{z z}$ bekannt und man erhält

$$
\tilde{\phi}(a, x):=C-\phi(x)=\frac{\mu}{\pi(1-v)} \int_{x}^{a} \frac{g^{\prime}(\xi)}{\xi} \mathrm{K}\left(\sqrt{1-\frac{x^{2}}{\xi^{2}}}\right) \mathrm{d} \xi, \quad c<|x| \leq a
$$

mit dem im Anhang definierten vollständigen elliptischen Integral erster Art, $\mathrm{K}(k)$. Die Konstante $C$ ergibt sich aus der Stetigkeit von $\phi$ an der Stelle $x=c$. Es ist

$$
\phi(x)=-\tilde{\phi}(a, x)+\tilde{\phi}(a, c), \quad c<|x| \leq a .
$$

Die Beziehung zwischen dem Verdrehwinkel $\varphi$ und den Radien $c$ und $a$ ist damit durch

$$
\varphi=\phi(a)=\tilde{\phi}(a, c)
$$

gegeben. Die Torsionsspannungen im Haftgebiet sind

$$
\sigma_{\varphi z}(r)=-\frac{4 G r}{\pi} \int_{c}^{a} \frac{\partial \tilde{\phi}(a, x)}{\partial x} \frac{\mathrm{d} x}{\sqrt{x^{2}-r^{2}}}, \quad r \leq c
$$

und das gesamte Torsionsmoment beträgt

$$
M_{z}=\frac{16 G}{3} \varphi c^{3}-4 \mu \int_{c}^{a}\left[c \sqrt{r^{2}-c^{2}}+r^{2} \arccos \left(\frac{c}{r}\right)\right] \sigma_{z z}(r) \mathrm{d} r .
$$

Die Ergebnisse in den Gl. (3.148), (3.149) und (3.150) wurden für einen allgemeinen rotationssymmetrischen Indenter zuerst von Jäger [10] publiziert. Für einen parabolischen Indenter mit dem Krümmungsradius $\tilde{R}$ fand Lubkin [66] die Lösung. Mit der Funktion $g(x)$ aus 
der Lösung des Normalkontaktproblems (siehe Gl. (3.34)) ergibt sich in diesem Fall als Lösung des Torsionsproblems

$$
\tilde{\phi}(a, x)=\frac{2 \mu a}{\pi(1-v) \tilde{R}}\left[\mathrm{~K}\left(\sqrt{1-\frac{x^{2}}{a^{2}}}\right)-\mathrm{E}\left(\sqrt{1-\frac{x^{2}}{a^{2}}}\right)\right],
$$

mit dem im Anhang definierten vollständigen elliptischen Integral zweiter Art E $(k)$. Die Spannung im Haftgebiet ist $[66,67]$

$$
\sigma_{\varphi z}(r)=\frac{8 \mu G a}{\pi^{2}(1-v) \tilde{R}} \sqrt{1-\frac{r^{2}}{a^{2}}}\left\{\frac{\pi}{2}+[\mathrm{K}(k)-\mathrm{E}(k)] \mathrm{F}\left(\beta, k^{\prime}\right)-\mathrm{K}(k) \mathrm{E}\left(\beta, k^{\prime}\right)\right\},
$$

mit

$$
k^{\prime}:=\frac{c}{a}, \quad k:=\sqrt{1-k^{\prime 2}}, \quad \beta:=\arcsin \sqrt{\frac{c^{2}-r^{2}}{c^{2}-\left(k^{\prime} r\right)^{2}}}
$$

sowie den im Anhang definierten unvollständigen elliptischen Integralen erster und zweiter Art, $\mathrm{F}(\beta, k)$ und $\mathrm{E}(\beta, k)$. Die Schubspannungsverteilung ist in normierter Darstellung für verschiedene Haftradien in Abb.3.8 gezeigt. Das gesamte Torsionsmoment wurde von Lubkin numerisch bestimmt und tabellarisch angegeben. Jäger [67] gab als analytische Näherung den Ausdruck

$$
M_{z} \approx \frac{4 \mu \tilde{E} a^{4}}{3 \tilde{R}} k^{2}\left(1-k^{2}+\frac{3 \pi}{16} k^{2}\right)
$$

an.

Abb. 3.8 Verteilung der normierten Schubspannungen ( $p_{0}$ bezeichnet den mittleren Druck) bei verschiedenen Haftradien für das Lubkin-Problem. Die dünne Linie bezeichnet die Verteilung bei vollständigem Gleiten

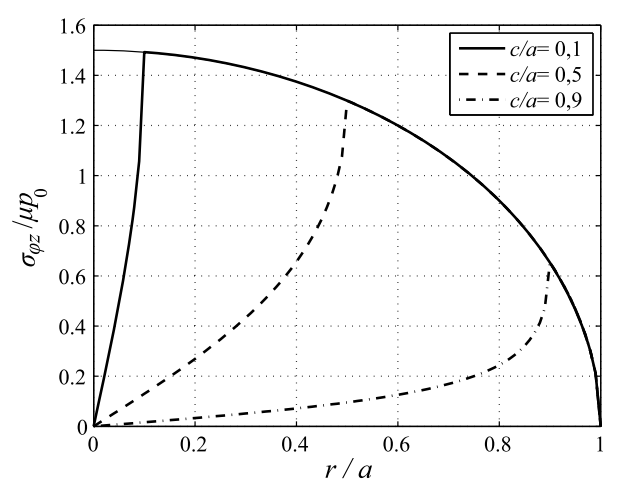




\subsubsection{Erweiterung auf beliebige Belastungsgeschichten (parabolischer Kontakt)}

Deresiewicz [68] untersuchte den oszillierenden Torsionskontakt von elastischen Kugeln. Eine allgemeine Lösung für beliebige Belastungsgeschichten wurde später von Jäger [67] angegeben. Diese basiert, analog zu dem entsprechenden Tangentialkontaktproblem, auf einer geeigneten Superposition von Lubkin-Lösungen

$$
\begin{aligned}
& \sigma_{L}(r ; a, c):=\frac{2 \mu \tilde{E}}{\pi \tilde{R}} \sqrt{a^{2}-r^{2}} \begin{cases}1+\frac{2}{\pi}\left\{[\mathrm{K}(k)-\mathrm{E}(k)] \mathrm{F}\left(\beta, k^{\prime}\right)-\mathrm{K}(k) \mathrm{E}\left(\beta, k^{\prime}\right)\right\}, & r \leq c, \\
1, & c<r \leq a .\end{cases} \\
& \varphi_{L}(a, c):=\frac{2 \mu a}{\pi(1-v) \tilde{R}}[\mathrm{~K}(k)-\mathrm{E}(k)],
\end{aligned}
$$

wobei die Definitionen von $k, k^{\prime}$ und $\beta$ den Gl. (3.153) entnommen werden können.

Man erkennt, dass durch eine Superposition $\sigma_{L}\left(r ; a_{i}, a_{k}\right)-\sigma_{L}\left(r ; a_{i}, a_{j}\right)$ mit $a_{i} \geq a_{j} \geq$ $a_{k}$ ein spannungsfreier Ring $a_{j} \leq r \leq a_{i}$ erzeugt werden kann, während das Gebiet $r \leq a_{k}$ haftet. Diese Beobachtung genügt, um die Spannungsverteilung für beliebige Belastungsgeschichten zu konstruieren. Da das Torsionskontaktproblem nicht, wie das Tangentialkontaktproblem in der Cattaneo-Mindlin-Näherung, auf das Normalkontaktproblem zurückgeführt werden kann und die Lubkin-Spannungen keine zu Gl. (3.109) analoge Superpositionsregel erfüllen, ist die Behandlung der einzelnen Szenarien (etwas paradoxerweise) dabei sogar einfacher als im Fall des Tangentialkontaktes.

Man betrachte einen Kontakt nach einer Lubkin-Belastung. Das Kontaktgebiet mit dem Radius $a_{1}$ besteht aus einem Haftgebiet mit dem Radius $c_{1}$ und einem Gleitgebiet $c_{1}<r$ $\leq a_{1}$. Ohne Beschränkung der Allgemeinheit sei angenommen, dass $\varphi_{1}>0$. Die Verteilung der Schubspannungen ist durch Gl. (3.155) gegeben. Nun wird die Eindrucktiefe um $\Delta d$ und anschließend die Starrkörperrotation um $\Delta \varphi$ verändert ${ }^{8}$. Da sich bei jeder (noch so kleinen) torsionalen Belastung vom Rand des Kontaktes ein Gleitgebiet ausbreitet, zerfällt das neue Kontaktgebiet mit dem Radius $a_{2}$ wiederum in ein Haftgebiet mit dem Radius $c_{2}$ und ein Gleitgebiet $c_{2}<r \leq a_{2}$. Grundsätzlich müssen zwei Fälle unterschieden werden: ein wachsendes Kontaktgebiet mit $a_{2}>a_{1}$ (also $\Delta d>0$ ) und ein schrumpfendes Kontaktgebiet mit $a_{2}<a_{1}$ (also $\Delta d<0$ ).

\section{Kontaktgebiet wächst}

Wenn das Kontaktgebiet wächst, gibt es zunächst - bevor die zusätzliche Verdrehung $\Delta \varphi$ aufgebracht wird - einen Zustand vollständigen Haftens. Danach breitet sich das Gleitgebiet erneut vom Rand des Kontaktes nach innen aus. Solange $c_{2}>a_{1}$, kann einfach eine einzelne Lubkin-Spannung linear superponiert werden,

\footnotetext{
${ }^{8}$ Wie im Tangentialkontakt müssen beide Inkremente nicht zwangsläufig infinitesimal sein.
} 


$$
\begin{aligned}
\sigma_{\varphi z}(r) & =\sigma_{L}\left(r ; a_{1}, c_{1}\right)+\sigma_{L}\left(r ; a_{2}, c_{2}\right) \operatorname{sgn}(\Delta \varphi), \\
\varphi & =\varphi_{L}\left(a_{1}, c_{1}\right)+\varphi_{L}\left(a_{2}, c_{2}\right) \operatorname{sgn}(\Delta \varphi) .
\end{aligned}
$$

Die Bedingung $c_{2}>a_{1}$ führt dabei auf die Forderung

$$
|\Delta \varphi|<\varphi_{L}\left(a_{2}, a_{1}\right)
$$

Falls $c_{1}<c_{2}<a_{1}$, muss zunächst der Ring $c_{2} \leq r \leq a_{1}$ von Spannungen befreit werden; anschließend wird wiederum eine einzelne Lubkin-Spannung linear superponiert:

$$
\begin{aligned}
\sigma_{\varphi z}(r) & =\sigma_{L}\left(r ; a_{1}, c_{1}\right)-\sigma_{L}\left(r ; a_{1}, c_{2}\right)+\sigma_{L}\left(r ; a_{2}, c_{2}\right) \operatorname{sgn}(\Delta \varphi), \\
\varphi & =\varphi_{L}\left(a_{1}, c_{1}\right)-\varphi_{L}\left(a_{1}, c_{2}\right)+\varphi_{L}\left(a_{2}, c_{2}\right) \operatorname{sgn}(\Delta \varphi) .
\end{aligned}
$$

Für den Fall $c_{2} \leq c_{1}$ wurde die ganze ursprüngliche Belastungsgeschichte gelöscht und die Lösung ist entsprechend einfach

$$
\begin{aligned}
\sigma_{\varphi z}(r) & =\sigma_{L}\left(r ; a_{2}, c_{2}\right) \operatorname{sgn}(\Delta \varphi), \\
\varphi & =\varphi_{L}\left(a_{2}, c_{2}\right) \operatorname{sgn}(\Delta \varphi) .
\end{aligned}
$$

Die Bestimmung der zu Gl.(3.159) analogen Bedingungen für $\Delta \varphi$ in den letzten beiden Fällen ist dabei elementar.

\section{Kontaktgebiet schrumpft}

Wenn das Kontaktgebiet schrumpft, muss zunächst der Ring $a_{2}<r \leq a_{1}$ von Schubspannungen befreit werden. Dazu ist eine Verteilung

$$
\begin{aligned}
\Delta \sigma_{\varphi z, I}(r) & =-\sigma_{L}\left(r ; a_{1}, a_{2}\right), \\
\Delta \varphi_{I} & =-\varphi_{L}\left(a_{1}, a_{2}\right)
\end{aligned}
$$

nötig. Anschließend kann man wie oben verfahren, wobei der Kontaktradius konstant bleibt.

\section{Allgemeine Ausgangssituation}

Die obigen Beziehungen kann man ohne Schwierigkeiten in iterativer Form für eine beliebige Ausgangssituation verallgemeinern. Es sei $\sigma_{\varphi z, n}$ die Spannungsverteilung nach $n$ Verschiebungsinkrementen $\Delta d_{i}$ und $\Delta \varphi_{i}$. Außerdem sei durch $s_{i}$ das Vorzeichen der jeweiligen Sliprichtung definiert. Dann ist

$$
\sigma_{\varphi z, n}(r)= \begin{cases}\sigma_{\varphi z, n-1}(r)+s_{n} \sigma_{L}\left(r ; a_{n}, c_{n}\right) & \text { für } 1 \\ \sigma_{\varphi z, n-1}(r)-s_{n-1} \sigma_{L}\left(r ; a_{n-1}, c_{n}\right)+s_{n} \sigma_{L}\left(r ; a_{n}, c_{n}\right), & \text { für } 2 \\ \sigma_{\varphi z, n-2}(r)+s_{n} \sigma_{L}\left(r ; a_{n}, c_{n}\right) & , \text { sonst, }\end{cases}
$$

wobei die Bedingungen durch 


$$
\begin{gathered}
\text { (1): } \Delta d_{n} \geq 0 \wedge\left|\Delta \varphi_{n}\right| \leq \varphi_{L}\left(a_{n}, a_{n-1}\right), \\
\text { (2): }\left(\Delta d_{n} \geq 0 \wedge \varphi_{L}\left(a_{n}, a_{n-1}\right)<\left|\Delta \varphi_{n}\right| \leq \varphi_{L}\left(a_{n}, c_{n-1}\right)-\varphi_{L}\left(a_{n-1}, c_{n-1}\right) \operatorname{sgn}\left(\Delta \varphi_{n}\right)\right) \\
\vee\left(\Delta d_{n}<0 \wedge \varphi_{L}\left(a_{n-1}, a_{n}\right)<-\Delta \varphi_{n} \leq \varphi_{L}\left(a_{n}, c_{n-1}\right)+\varphi_{L}\left(a_{n-1}, c_{n-1}\right)\right)
\end{gathered}
$$

gegeben sind. Wenn der sonstige Fall in Gl. (3.166) eintritt, wurde offenbar das Inkrement mit dem Index $n-1$ vollständig gelöscht. Man muss dann den Index um Eins reduzieren.

\subsection{Viskoelastizität}

\subsubsection{Einführung}

Elastomere sind sehr vielseitige Werkstoffe. Durch ihre hohe Deformierbarkeit in Verbindung mit einem vergleichsweise kleinen Elastizitätsmodul passen sie sich sehr gut an andere Oberflächen an. Sie haben ein je nach Zeitskala unterschiedliches Materialverhalten, das auch ihre Kontakteigenschaften maßgeblich beeinflusst. Dabei weist Gummi in vielen Materialpaarungen einen hohen Reibbeiwert im nutzbaren Zeitbereich auf. Außerdem sind Elastomere beständig gegenüber Hitze und Feuchtigkeit. Aufgrund dieser Vielseitigkeit finden Elastomere in Reifen, Dichtungen und anderen technischen Systemen häufige Anwendung. Auch Biomaterialien wie Gelenkknorpel kann man oft als (mehrphasige) viskoelastische Medien modellieren.

Das Materialverhalten dieser Werkstoffklasse lässt sich grob in statische und dynamische Eigenschaften unterteilen. Beide haben ihren Ursprung in der molekularen Struktur der Elastomere, die aus schwach miteinander wechselwirkenden langen Polymerketten aufgebaut sind. Im thermodynamischen Gleichgewicht ist die Anzahl möglicher Konfigurationen einer einzelnen Kette - und damit ihre Entropie - abhängig vom Abstand zwischen den beiden Kettenenden. Im spannungsfreien Zustand befindet sich die Kette im bevorzugten ,,verknäuelten“ Zustand maximaler Entropie. Legt man an einen Elastomerblock daher quasistatisch eine Spannung an, werden die Polymerketten ,entflechtet“ und die Entropie sinkt. Da damit die Freie Energie steigt, ergibt sich eine elastische Reaktion, die der angelegten Spannung entgegenwirkt. Der mit dieser entropieinduzierten Elastizität verbundene Elastizitätsmodul ist sehr klein (in der Regel von der Größenordnung $1 \mathrm{MPa}$ ) und der ganze Prozess mehr oder weniger frei von Dissipation ${ }^{9}$.

Die Relaxation in das thermodynamische Gleichgewicht kann allerdings je nach der konkreten Struktur des Elastomers unterschiedlich viel Zeit in Anspruch nehmen. Instantan (das heißt im Moment des Anlegens der Spannung) reagiert das Elastomer wie ein Festkörper

\footnotetext{
${ }^{9}$ Tatsächlich zeigen viele Elastomere eine schwache „statische Hysterese“. Inwieweit diese auf viskose Verluste bei sehr kleinen Geschwindigkeiten zurückzuführen ist, soll an dieser Stelle nicht diskutiert werden. Der genannte Effekt wird im weiteren Verlauf dieses Buches auch nicht berücksichtigt.
} 
mit einem Elastizitätsmodul der Größenordnung $1 \mathrm{GPa}$. Dieser „Glasmodul“ ist also um mehrere Größenordnungen höher als der oben beschriebene statische Modul. Durch die innere Reibung während der Relaxation ist die Deformation eines Elastomers auf mittleren Zeitskalen darüber hinaus mit teilweise hoher Energie-Dissipation verbunden.

Insbesondere bei großen Deformationen verhalten sich Elastomere nichtlinear. Im Rahmen dieses Buches sollen sie allerdings als linear-viskoelastische Medien betrachtet werden, weil die Berücksichtigung von Nichtlinearitäten sehr kompliziert ist und den Rahmen dieses Buches sprengen würde. Im folgenden Abschnitt wird daher das allgemeine linear-viskoelastische Materialgesetz von Elastomeren eingeführt, auf dem deren kontaktmechanische Eigenschaften beruhen. Anschließend werden rheologische Modelle und die Kontaktmechanik von viskoelastischen Medien untersucht.

\subsubsection{Das allgemeine linear-viskoelastische Materialgesetz}

Man betrachte zunächst nur die reine Schubdeformation eines Elastomers: Wird ein Elastomerblock um einen Winkel $2 \varepsilon$ geschert und diese Deformation aufrecht erhalten, relaxiert die Schubspannung in dem Medium wegen der oben beschriebenen Mechanismen nach einer Funktion $\sigma=\sigma(t)$. Den auf die Deformation bezogenen Ausdruck

$$
G(t):=\frac{\sigma(t)}{2 \varepsilon}
$$

bezeichnet man als zeitabhängigen Schubmodul. Analog dazu gibt es den statischen Kriechversuch, bei dem eine konstante Scherspannung $\sigma$ an den Block angelegt wird. Die Deformation vergrößert sich dann im Laufe der Zeit, $\varepsilon=\varepsilon(t)$, und den auf die angelegte Spannung bezogenen Ausdruck

$$
W(t):=\frac{2 \varepsilon(t)}{\sigma}
$$

nennt man Kriechfunktion. Setzt man lineares Materialverhalten voraus, können die Spannungen bei einer beliebigen Deformationsgeschichte durch die Superposition

$$
\sigma(t)=2 \int_{-\infty}^{t} G\left(t-t^{\prime}\right) \dot{\varepsilon}\left(t^{\prime}\right) \mathrm{d} t^{\prime}
$$

bestimmt werden. Durch Laplace-Transformation dieser Gleichung erhält man unter der Annahme, dass das Medium bis zum Zeitpunkt $t=0$ undeformiert und spannungsfrei war,

$$
\sigma^{*}(s)=2 s G^{*}(s) \varepsilon^{*}(s)
$$


wobei hier und im Folgenden der Hochstern die Laplace-Transformierte einer zeitabhängigen Funktion bezeichnet. Analog ergibt sich die Deformation bei einer allgemeinen Belastungsgeschichte zu

$$
\varepsilon(t)=\frac{1}{2} \int_{-\infty}^{t} W\left(t-t^{\prime}\right) \dot{\sigma}\left(t^{\prime}\right) \mathrm{d} t^{\prime}
$$

was im Laplace-Raum (die Belastung beginne wiederum erst bei $t=0$ ) dem Produkt

$$
\varepsilon^{*}(s)=\frac{s}{2} W^{*}(s) \sigma^{*}(s),
$$

entspricht. Aus dem Vergleich der Gl. (3.172) und (3.174) wird sofort deutlich, dass der zeitabhängige Schubmodul (manchmal auch Relaxationsfunktion genannt) und die Kriechfunktion nicht unabhängig voneinander sind. Sie bilden ein Transformationspaar, das der Gleichung

$$
s^{2} G^{*}(s) W^{*}(s) \equiv 1
$$

gehorcht. Eine weitere Materialfunktion, die häufig zur Beschreibung des viskoelastischen Verhaltens von Elastomeren verwendet wird, ist der Komplexe (frequenzabhängige) Schubmodul $\hat{G}(\omega)$. Dieser beschreibt die Spannungsantwort bei harmonischer Dehnung und kann über die Beziehung

$$
\hat{G}(\omega):=i \omega G^{*}(s=i \omega)
$$

mit der imaginären Einheit $i$, aus der Laplace-Transformierten des zeitabhängigen Moduls gewonnen werden. Sein Realteil wird Speichermodul und sein Imaginärteil Verlustmodul genannt.

Vereinzelt trifft man in der Literatur auch die Funktionen $s G^{*}(s)$ und $s W^{*}(s)$ als eigenständige Transformierte der Materialfunktionen. Das hat zum einen den Vorteil, dass diese Funktionen die gleichen physikalischen Einheiten aufweisen wie ihre zeitabhängigen Ursprünge, $G(t)$ und $W(t)$. Zum anderen ist das Produkt der beiden Transformierten wegen Gl. (3.175) grundsätzlich gleich Eins.

Im Allgemeinen zeigt ein Elastomer auch eine viskoelastische Reaktion gegenüber hydrostatischer Kompression. Völlig analog zu den obigen Betrachtungen zur Schubbelastung kann man in diesem Fall einen zeitabhängigen Kompressionsmodul $K(t)$ und eine entsprechende Kriechfunktion einführen. Das vollständige allgemeine linear-viskoelastische Materialgesetz (bereits in nach Scher- und Spuranteil aufgeteilter Form) lautet damit wie folgt: 


$$
\begin{aligned}
& \Sigma_{i j}(t)=2 \int_{-\infty}^{t} G\left(t-t^{\prime}\right) \dot{e}_{i j}\left(t^{\prime}\right) \mathrm{d} t^{\prime}, \\
& \sigma_{l l}(t)=3 \int_{-\infty}^{t} K\left(t-t^{\prime}\right) \dot{\varepsilon}_{l l}\left(t^{\prime}\right) \mathrm{d} t^{\prime} .
\end{aligned}
$$

Dabei bezeichnen $\Sigma_{i j}$ und $e_{i j}$ die spurfreien Anteile des Spannungs- und Verzerrungstensors. Die Berücksichtigung des Kriechens bei hydrostatischer Kompression verkompliziert die Lösung von Kontaktaufgaben mit Elastomeren in der Regel deutlich, wie z. B. anhand der Lösung eines noch vergleichsweise einfachen Problems der konischen Indentierung mit einem monoton wachsenden Kontaktradius und rein elastischem Verhalten bei hydrostatischer Kompression von Vandamme und Ulm [69] deutlich wird. In der Regel verwendet man daher nur die Reaktion gegen reinen Schub zur Charakterisierung eines Elastomers. Dies ist sehr häufig auch durchaus berechtigt, da man die meisten relevanten Elastomere, wie Gummi oder Gelenkknorpel, in guter Näherung als inkompressibel annehmen kann [70, 71]. Trotzdem besteht aber natürlich keine physikalische Notwendigkeit, dass ein Elastomer keine Deformationsantwort gegen hydrostatische Kompression aufweist; so nimmt man beispielsweise in der Geophysik an, dass der Erdmantel aus kompressiblem viskoelastischem Material aufgebaut ist [72]. Im folgenden Abschnitt wird daher die Berücksichtigung der Kompressibilität für den Normalkontakt genauer beschrieben.

\subsubsection{Berücksichtigung der Kompressibilität (Normalkontakt)}

Für den reibungsfreien Normalkontakt kann man das kompressible Problem auf ein entsprechend modifiziertes inkompressibles Problem zurückführen. Man benötigt dafür nur die Fundamentallösung des elastischen Normalkontaktproblems und das viskoelastische Korrespondenzprinzip, das in spezieller Form zuerst von Alfrey [73] publiziert und später von Lee [74] und Radok [75] verallgemeinert wurde. Die genannten Arbeiten beziehen sich auf isotrope, homogene Medien.

Die Fundamentallösung für die vertikale Verschiebung der Oberfläche eines elastischen Halbraums unter Wirkung einer ab dem Zeitpunkt $t=0$ im Koordinatenursprung aufgebrachten Punktlast in $z$-Richtung ist nach Gl.(3.3) durch

$$
u_{z}^{\mathrm{el}}(r, t)=\frac{F_{z} \mathrm{H}(t)(1-v)}{2 \pi G r}=\frac{F_{z} \mathrm{H}(t)}{4 \pi G r} \frac{3 K+4 G}{3 K+G}=\frac{F_{z} \mathrm{H}(t)}{4 \pi G r}\left(1+\frac{3 G}{3 K+G}\right)
$$

gegeben, wobei $\mathrm{H}(t)$ die Heaviside-Funktion bezeichnet. Da das entsprechende viskoelastische Problem die gleichen Randbedingungen aufweist, kann es durch LaplaceTransformation problemlos in das elastische Problem mit einem Parameter $s$ 
überführt werden. Die viskoelastische Fundamentallösung kann man daher erhalten, indem zunächst mithilfe der Substitutionen des Korrespondenzprinzips ${ }^{10}$,

$$
\begin{aligned}
& G \rightarrow s G^{*}(s), \\
& K \rightarrow s K^{*}(s),
\end{aligned}
$$

und Laplace-Transformation der zeitabhängigen Punktlast die Laplace-Transformierte der viskoelastischen Lösung gewonnen wird:

$$
u_{z}^{*}(r, s)=\frac{F_{z}}{4 \pi r}\left\{\frac{1}{s^{2} G^{*}(s)}+\frac{3}{\left[G^{*}(s)+3 K^{*}(s)\right] s^{2}}\right\} .
$$

Die Rücktransformation von Gl. (3.182) liefert dann die gesuchte Lösung des viskoelastischen Problems,

$$
u_{z}(r, t)=\frac{F_{z}}{4 \pi r}\left[W(t)+W_{1}(t)\right]
$$

mit

$$
W_{1}^{*}(s):=\frac{3}{\left[G^{*}(s)+3 K^{*}(s)\right] s^{2}} \quad \Leftrightarrow \quad G_{1}(t)=K(t)+\frac{G(t)}{3} .
$$

Gl.(3.183) beschreibt aber die Fundamentallösung für einen inkompressiblen viskoelastischen Halbraum mit der Scher-Kriechfunktion $W(t)+W_{1}(t)$. Gelingt es also, die Gl. (3.184) nach $W_{1}(t)$ aufzulösen, ist das kompressible Normalkontaktproblem auf ein entsprechendes äquivalentes inkompressibles Problem zurückgeführt. Diese Rücktransformation ist allerdings in der Regel nur schwer analytisch durchzuführen. Es sei außerdem noch einmal darauf hingewiesen, dass diese Art der Berücksichtigung der Kompressibilität auf den normalen Fundamentallösungen des kompressiblen und des äquivalenten inkompressiblen Mediums beruht und daher nur für den reibungsfreien Normalkontakt anwendbar ist.

\subsubsection{Rheologische Modelle}

Eine weitere, häufig verwendete Möglichkeit zur Beschreibung der viskoelastischen Eigenschaften von Elastomeren sind sogenannte ,rheologische Modelle“, die aus einzelnen linearelastischen und linear-viskosen Elementen aufgebaut sind. Durch verschiedene Schaltungen dieser beiden Grundelemente kann man sehr unterschiedliches (und durch eine genügende Verallgemeinerung beliebiges) lineares viskoelastisches Materialverhalten repräsentieren. Zur Darstellung der elastischen und viskosen Elemente werden in der Regel Federn und

\footnotetext{
${ }^{10}$ Den in Gl.(3.172) gegebenen Zusammenhang kann man als elastisches Materialgesetz mit dem Parameter $s$ und dem Schubmodul $s G^{*}(s)$ deuten; das gleiche gilt auch für die LaplaceTransformation von Gl. (3.178).
} 
Dämpfer verwendet; man muss dabei aber in Erinnerung behalten, dass es sich um kontinuumsmechanische, volumenspezifische Größen handelt. Anstatt von Steifigkeiten und Dämpfungskonstanten wird im Folgenden daher von elastischen Modulen und Viskositäten die Rede sein.

Bis auf ausdrückliche Ausnahmen sind alle betrachteten Medien inkompressibel. Entsprechend bezieht sich die Darstellung nur auf die Rheologie bei der reinen Schubbelastung.

\section{Das Kelvin-Voigt-Medium}

Die in vieler Hinsicht einfachste Behandlung von viskoelastischem Materialverhalten besteht in der vollständigen Trennung der elastischen und viskosen Eigenschaften. Das rheologische Modell eines solchen, üblicherweise als Kelvin-Voigt-Körper bezeichneten Mediums ist eine Parallelschaltung einer Feder mit einem Dämpfer. Das Materialgesetz hat daher die Form

$$
\sigma(t)=2 G_{\infty} \varepsilon(t)+2 \eta \dot{\varepsilon}(t)
$$

Aus dem Vergleich der Gl.(3.171) und (3.185) liest man den zeitabhängigen Schubmodul ab,

$$
G(t)=G_{\infty}+\eta \delta(t)
$$

wobei $\delta(\cdot)$ die Dirac-Distribution bezeichnet. Daraus ist ersichtlich, dass dieses Material eine unendlich schnelle Spannungsrelaxation aufweist. Aus Gl. (3.176) ergibt sich außerdem

$$
\hat{G}(\omega)=G_{\infty}+i \eta \omega
$$

für den komplexen Schubmodul. Durch die Anwendung von Gl.(3.175) erhält man als Kriechfunktion den Ausdruck

$$
W(t)=\frac{1}{G_{\infty}}\left[1-\exp \left(-\frac{G_{\infty}}{\eta} t\right)\right] .
$$

\section{Das Maxwell-Medium}

Ein weiteres typisches rheologisches Modell ist das Maxwell-Medium, das durch eine Reihenschaltung aus einer Feder und einem Dämpfer repräsentiert wird. Die Superposition der Kriechfunktionen dieser beiden Grundbausteine liefert für die Kriechfunktion des MaxwellMediums den Ausdruck

$$
W(t)=\frac{1}{G_{1}}+\frac{t}{\eta_{1}} .
$$

Man erkennt, dass das viskoelastische Materialverhalten des Maxwell-Mediums keinen rein elastischen Anteil besitzt (das Material besitzt also keinen statischen Modul), da der Körper 
bei einer konstanten Belastung unbegrenzt kriecht. Das Material verhält sich auf sehr großen Zeitskalen daher wie eine Flüssigkeit. Die Relaxationsfunktion ergibt sich zu

$$
G(t)=G_{1} \exp \left(-\frac{G_{1}}{\eta_{1}} t\right) .
$$

Die rheologischen Modelle des Kelvin-Voigt-Körpers sowie des Maxwell-Körpers sind schematisch in Abb. 3.9 gezeigt.

\section{Ein Standardmodell für Gummi}

Die charakteristischen Eigenschaften von Gummi, die ein rheologisches Modell abbilden sollte, um ein zumindest qualitativ korrektes Materialverhalten zu erzielen, wurden bereits im einführenden Text zu diesem Unterkapitel aufgeführt:

- auf sehr kleinen Zeitskalen einen großen Schubmodul ohne Dissipation

- auf sehr großen Zeitskalen einen kleinen Schubmodul ohne nennenswerte Dissipation

- auf mittleren Zeitskalen hohe Dissipation

Es gibt verschiedene Möglichkeiten, ein minimales rheologisches Modell zu konstruieren, das diese Eigenschaften erfüllt. Eine Variante besteht darin, ein Maxwell-Element mit einer einzelnen Feder mit dem Modul $G_{\infty} \ll G_{1}$ parallel zu schalten. Das Medium verhält sich dann auch auf großen Zeitskalen wie ein Festkörper. Die Relaxations- und Kriechfunktion ergeben sich $\mathrm{zu}$

$$
\begin{aligned}
G(t) & =G_{\infty}+G_{1} \exp \left(-\frac{G_{1}}{\eta_{1}} t\right) \\
W(t) & =\frac{1}{G_{\infty}}\left[1-\frac{G_{1}}{G_{\infty}} \exp \left(-\frac{G_{\infty} G_{1} t}{\left(G_{1}+G_{\infty}\right) \eta_{1}}\right)\right],
\end{aligned}
$$

und der komplexe Schubmodul beträgt

$$
\hat{G}(\omega)=G_{\infty}+G_{1} \frac{(\omega \tau)^{2}}{1+(\omega \tau)^{2}}+i G_{1} \frac{\omega \tau}{1+(\omega \tau)^{2}}, \quad \tau:=\frac{\eta_{1}}{G_{1}} .
$$

\section{Abb. 3.9 Das}

Kelvin-Voigt-Medium (links) als rheologisches Modell sowie das Maxwell-Element (rechts)
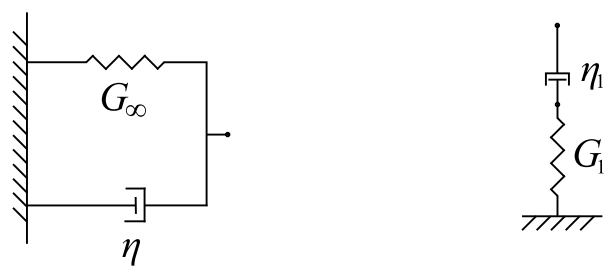
Für $G_{\infty} \rightarrow 0$ ergeben sich natürlich die Ausdrücke des Maxwell-Mediums, für $G_{1} \rightarrow \infty$ hingegen die des Kelvin-Voigt-Körpers.

\section{Prony-Reihen}

Das oben beschriebene Standard-Medium weist nur auf einer einzigen charakteristischen Zeitskala $\tau$ Spannungsrelaxation auf. Die Relaxation durch die „Entflechtung“ der Polymermoleküle ist aber in Wirklichkeit ein auf mehreren Längen- und Zeitskalen ablaufender Prozess. Ein reales Elastomer weist daher mehrere charakteristische Relaxationszeiten auf. Um dies in einem rheologischen Modell abzubilden, konstruiert man sogenannte „PronyReihen“. Es kommen dafür verallgemeinerte Maxwell- oder verallgemeinerte Kelvin-VoigtElemente in Frage. Bei einem verallgemeinerten Maxwell-Modell wird das beschriebene Standardmodell um eine beliebige Anzahl von Maxwell-Elementen mit den Modulen $G_{k}$ und den Viskositäten $\eta_{k}$ in Parallelschaltung ergänzt. Der gesamte zeitabhängige Modul ist dann

$$
G(t)=G_{\infty}+\sum_{k=1}^{N} G_{k} \exp \left(-\frac{t}{\tau_{k}}\right), \quad \tau_{k}:=\frac{\eta_{k}}{G_{k}}
$$

Durch den Übergang zu einem kontinuierlichen Spektrum ergibt sich die Transformation

$$
G(t)=G_{\infty}+\int_{0}^{\infty} g(s) \exp (-s t) \mathrm{d} s
$$

durch die sich wegen der Existenz und Eindeutigkeit der Inversen Laplace-Transformation beliebiges linear-viskoelastisches Materialverhalten in dem rheologischen Modell abbilden lässt. Technische Pronyreihen nutzen in der Regel 15 bis 30 verschiedene logarithmisch skalierte Relaxationszeiten.

\section{Der Kelvin-Maxwell-Körper}

Wenn das betrachtete Kontaktproblem eine eigene Zeitskala $\tau$ hat, z. B. eine charakteristische Dauer ${ }^{11}$, spielen die Relaxationsprozesse, die auf eben dieser Zeitskala ablaufen eine dominierende Rolle. Alle anderen Relaxationen können, zumindest in guter Näherung, entweder als unendlich langsam oder als unendlich schnell betrachtet werden. Die verschiedenen Maxwell-Elemente der Prony-Reihe mit großen Relaxationszeiten kann man daher in diesem Fall zu einer einzelnen Feder mit dem Modul $G_{\infty}$ (gemeinsam mit dem tatsächlichen statischen Modul) zusammenfassen, während die Elemente mit sehr kleinen Relaxationszeiten in einem einzelnen Dämpfer mit der Viskosität $\eta_{0}$ aufgehen:

\footnotetext{
${ }^{11}$ Bei Stoßproblemen hat diese charakteristische Dauer in der Regel die Größenordnung von einigen Millisekunden.
} 

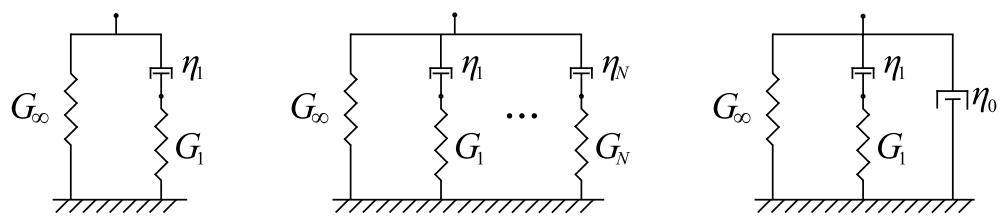

Abb. 3.10 Das Standardelement (links), eine Prony-Reihe in Form eines verallgemeinerten Maxwell-Elements (mitte) und ein Kelvin-Maxwell-Element (rechts). Die verwendeten Größen können dem Text entnommen werden

$$
\begin{aligned}
G_{\infty} & =\sum_{\tau_{k} \gg \tau} G_{k}, \\
\eta_{0} & =\sum_{\tau_{k} \ll \tau} \eta_{k} .
\end{aligned}
$$

Es entsteht dann eine Parallelschaltung aus einem Kelvin-Voigt- mit einem MaxwellElement, dessen Relaxationszeit genau der Zeitskala $\tau$ entspricht. Die gesamte Relaxationsfunktion dieses Kelvin-Maxwell-Körpers ist elementarerweise durch

$$
G(t)=G_{\infty}+G_{1} \exp \left(-\frac{G_{1}}{\eta_{1}} t\right)+\eta_{0} \delta(t), \quad \eta_{1}=G_{1} \tau,
$$

gegeben. Dieses rheologische Element besitzt nur drei freie Parameter (die Zeitskala $\tau$ ist durch das Kontaktproblem vorgegeben) und ist deswegen sehr einfach zu implementieren und zu untersuchen. Andererseits ist das Modell gleichzeitig sehr allgemein, da es aus einer beliebig komplizierten Prony-Reihe hervorgeht.

Abb. 3.10 zeigt schematische Darstellungen der rheologischen Modelle des Standardkörpers und des beschriebenen Kelvin-Maxwell-Körpers sowie eine Prony-Reihe in Form eines verallgemeinerten Maxwell-Modells.

\section{Das rheologische Modell für ein allgemeines kompressibles Medium}

Es soll zum Abschluss der Betrachtung rheologischer Modelle noch einmal kurz auf den Kontakt mit kompressiblen Medien eingegangen werden, allerdings ohne eine spezielle Rheologie zu berücksichtigen, das heißt, für ein beliebiges Relaxationsverhalten bei Scherung oder Kompression, ausgedrückt durch die beiden Materialfunktionen $G(t)$ und $K(t)$.

Während die analytische Rücktransformation von Gl. (3.184) im Allgemeinen sehr schwierig sein kann, ist es sehr einfach, das rheologische Modell zu konstruieren, das die äquivalente Scher-Kriechfunktion $W(t)+W_{1}(t)$ aufweist, welche nötig ist, um das kompressible Medium für den Normalkontakt durch ein äquivalentes inkompressibles Medium zu ersetzen: es ist einfach eine Reihenschaltung der rheologischen Modelle, welche die Relaxationsfunktionen $G(t)$ und $G_{1}(t)$ reproduzieren. Dabei ist wegen Gl. (3.184) $G_{1}(t)$ eine einfache Superposition, also Parallelschaltung, der Relaxationen $K(t)$ und $G(t) / 3$. Das vollständige rheologische Modell ist in Abb.3.11 gezeigt. 
Abb.3.11 Das rheologische Modell für ein kompressibles Medium mit beliebiger Rheologie (nach [76]). Die Boxen sind als verallgemeinerte rheologische Elemente zu verstehen, die das angegebene Relaxationsverhalten reproduzieren

\subsubsection{Behandlung viskoelastischer Kontaktprobleme nach Lee und Radok}

Mithilfe des Korrespondenzprinzips zwischen Randwertproblemen der linearen Elastizität und Viskoelastizität kann man unter bestimmten Umständen auch Kontaktprobleme zwischen viskoelastischen Körpern untersuchen, wie zuerst Lee und Radok [77] zeigen konnten.

Man betrachte den Normalkontakt zwischen einem starren axialsymmetrischen konvexen Indenter mit dem Profil $f(r)$ und einem inkompressiblen viskoelastischen Medium ${ }^{12}$ mit dem zeitabhängigen Schubmodul $G(t)$. Dann lautet die normierte Spannungsverteilung für das entsprechende elastische Problem mit dem Kontaktradius $a$ nach Gl. (3.28) wie folgt:

$$
\sigma_{z z}^{\mathrm{el}}(r, t)=\sigma_{z z}^{\mathrm{el}}(r ; a(t)):=-\frac{4}{\pi} \int_{r}^{a(t)} \frac{g^{\prime}(x)}{\sqrt{x^{2}-r^{2}}} \mathrm{~d} x, \quad r \leq a(t),
$$

wobei die Hilfsfunktion $g(x)$ durch Gl. (3.32) gegeben ist. Die nach dem Korrespondenzprinzip zugehörige viskoelastische Lösung lautet im Laplace-Raum (der Stern bezeichnet wiederum die jeweilige Laplace-Transformierte)

$$
\sigma_{z z}^{*}(r, s)=s G^{*}(s)\left(\sigma_{z z}^{\mathrm{el}}\right)^{*}(r, s),
$$

und im Zeitbereich

$$
\sigma_{z z}(r, t)=\int_{0}^{t} G\left(t-t^{\prime}\right) \frac{\partial \sigma_{z z}^{\mathrm{el}}}{\partial t^{\prime}}\left(r, t^{\prime}\right) \mathrm{d} t^{\prime}
$$

Lee \& Radok konnten zeigen, dass die Druckverteilung (3.201) tatsächlich die gemischten Randbedingungen des viskoelastischen Kontaktproblems erfüllt, falls der Kontaktradius

\footnotetext{
${ }^{12} \mathrm{Da}$ beide Kontaktpartner elastisch ähnlich sind, muss der Kontakt nicht notwendigerweise reibungsfrei sein.
} 
$a(t)$ monoton mit der Zeit wächst. Der Beweis von Lee \& Radok bezieht sich auf den parabolischen Kontakt, lässt sich aber ohne Schwierigkeiten für beliebige axialsymmetrische Profile verallgemeinern [78].

Im Fall des monoton wachsenden Kontaktradius ist außerdem der Zusammenhang zwischen Eindrucktiefe und Kontaktradius universal,

$$
d(t)=d^{\mathrm{el}}(t)=d^{\mathrm{el}}(a(t))=g(a(t)) .
$$

Die gesamte Normalkraft ergibt sich zu

$$
F_{z}(t)=\int_{0}^{t} G\left(t-t^{\prime}\right) \frac{\mathrm{d} F_{z}^{\mathrm{el}}}{\mathrm{d} t^{\prime}}\left(t^{\prime}\right) \mathrm{d} t^{\prime}
$$

wobei wegen Gl.(3.27) die entsprechende elastische Lösung durch

$$
F_{z}^{\mathrm{el}}(t)=F_{z}^{\mathrm{el}}(a(t)):=-8 \int_{0}^{a(t)}[g(a(t))-g(x)] \mathrm{d} x .
$$

gegeben ist.

\subsubsection{Erweiterung auf beliebige Belastungsgeschichten}

Schon Lee \& Radok haben erkannt, dass die Anwendung der oben beschriebenen Methode für Fälle, in denen der Kontaktradius ein Maximum besitzt, zu unphysikalischen Zugspannungen in den Gebieten am Rand des Kontaktes führt, in denen im Laufe der Indentierung der Kontakt wieder verloren geht. Die korrekte Lösung des axialsymmetrischen Kontaktproblems stammt in diesem Fall von Graham [78] und Ting [79]. Hunter [80] publizierte bereits 1960 die Lösung für den parabolischen Kontakt und untersuchte damit das viskoelastische Normalstoßproblem von Kugeln. Eine alternative aber äquivalente Formulierung schlug später Greenwood [81] vor.

Der Zeitpunkt, bei dem der Kontaktradius sein Maximum annimmt sei $t_{m}$. Dann kann für alle $t \leq t_{m}$ das Kontaktproblem mit den obigen Gleichungen gelöst werden. Für alle $t^{\prime}>t_{m}$ gibt es nun einen Zeitpunkt $t_{1}\left(t^{\prime}\right)<t_{m}$ (siehe die erläuternde Skizze in Abb.3.12), sodass

$$
a\left(t_{1}\right)=a\left(t^{\prime}\right)
$$

Die korrekten Ausdrücke für die Eindrucktiefe und die Normalkraft für $t>t_{m}$ lauten dann nach Ting wie folgt: 
Abb. 3.12 Beispielhafter Verlauf des Kontaktradius mit einem einzelnen Maximum

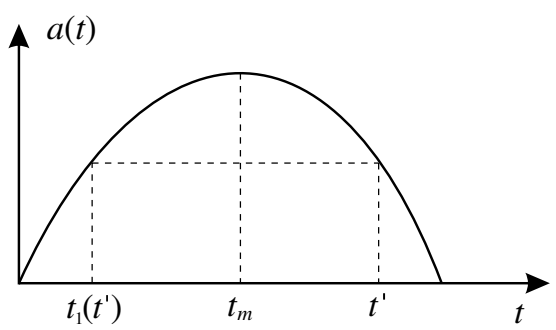

$d(t)=d^{\mathrm{el}}(a(t))-\int_{t_{m}}^{t} W\left(t-t^{\prime}\right) \frac{\mathrm{d}}{\mathrm{d} t^{\prime}}\left[\int_{t_{1}\left(t^{\prime}\right)}^{t^{\prime}} G\left(t^{\prime}-t^{\prime \prime}\right) \frac{\mathrm{d} d^{\mathrm{el}}}{\mathrm{d} t^{\prime \prime}}\left(t^{\prime \prime}\right) \mathrm{d} t^{\prime \prime}\right] \mathrm{d} t^{\prime}$,

$\sigma_{z z}(r, t)=\int_{0}^{t_{1}(t)} G\left(t-t^{\prime}\right) \frac{\partial \sigma_{z z}^{\mathrm{el}}}{\partial t^{\prime}}\left(r, t^{\prime}\right) \mathrm{d} t^{\prime}$,

$$
F_{z}(t)=\int_{0}^{t_{1}(t)} G\left(t-t^{\prime}\right) \frac{\mathrm{d} F_{z}^{\mathrm{el}}}{\mathrm{d} t^{\prime}}\left(t^{\prime}\right) \mathrm{d} t^{\prime}
$$

mit der Kriechfunktion $W(t)$ des viskoelastischen Mediums.

Eine ähnliche Prozedur kann man auch entwickeln, wenn der Kontaktradius eine beliebige Anzahl von Maxima und Minima aufweist, wie in späteren Publikationen von Graham [82] und Ting [83] demonstriert wurde. Allerdings wird die Ausführung der verketteten Differentiationen und Integrationen mit jedem Extremum des Kontaktradius mühseliger. Da für die Behandlung des einfachen Stoßproblems der Fall eines einzigen Maximums ausreicht, soll an dieser Stelle auf die Angabe der allgemeinen Gleichungen verzichtet werden.

\subsection{Funktionale Gradientenmedien}

\subsubsection{Einführung}

Angetrieben durch den technologischen Bedarf nach größerer Beständigkeit und flexiblerer Einsetzbarkeit von Werkstoffen und nicht zuletzt beflügelt durch das Studium von Lösungen, die die Natur in biologischen Tribosystemen entwickelt hat, wurde in den vergangenen Jahrzehnten der Kontaktmechanik von komplexeren Materialklassen - wie Verbundwerkstoffen, geschichteten Medien oder Funktionalen Gradientenmaterialien (FGM) - ein hohes $\mathrm{Ma} ß$ wissenschaftlicher Aufmerksamkeit zuteil. Da, wie sich herausstellt, die Verwendung von FGM in stoßbeanspruchten Systemen von großem Vorteil sein kann, z. B. zur Reduktion der auftretenden Kontaktspannungen, ist das vorliegende Unterkapitel der Kontaktmechanik solcher Medien gewidmet. 
Innerhalb eines FGM variieren die mechanischen Eigenschaften kontinuierlich über das Volumen, das Material ist inhomogen. Beispiele sind gehärtete Oberflächen sowie vielfältige biologische und biotechnologische Systeme, wie Knochen, Gelenke oder deren künstliche Varianten, Haftvorrichtungen (z. B. an den Füßen von Geckos) und Zellmembranen [84]. Ein korrekt eingestellter Gradient des Elastizitätsmoduls kann nachweislich zu erhöhter Verschleiß-Beständigkeit führen [85]. Im Gegensatz zu der in vielerlei Hinsicht ähnlichen Klasse der geschichteten Medien leiden FGM dabei nicht unter Delamination, thermozyklischem Kriechen oder anderen an diskrete Grenzflächen gebundenen Versagensmechanismen.

Im Fokus dieses Buches stehen Medien, in denen der Schubmodul $G$ mit der Tiefe $z$ variiert (bei zumindest näherungsweise konstanter Poissonzahl). Diese spezielle Form der Inhomogenität untersuchten Geomechaniker schon seit den 1940er Jahren, lange bevor überhaupt der Begriff „Funktionale Gradientenmaterialien“ geprägt wurde, siehe beispielsweise die Arbeiten von Holl [86], Rostovtsev [87] und Gibson [88]. Systematische Untersuchungen des Normalkontaktes ohne Adhäsion gehen auf Booker et al. [89, 90] und Giannakopoulos und Suresh [91, 92]) zurück. Im Laufe der Zeit wurden unterschiedliche konkrete Funktionen $G=G(z)$ betrachtet (siehe z. B. die Abhandlung von Selvadurai [93]); der einzige Fall, der eine weitgehend analytische Behandlung zulässt, scheint dabei das Potenzgesetz

$$
G(z)=G_{0}\left(\frac{z}{z_{0}}\right)^{k}, \quad|k|<1,
$$

zu sein. Während der Definitionsbereich des Exponenten $k$ in den meisten Arbeiten auf nicht-negative Werte beschränkt wird, geht aus der Arbeit von Fabrikant und Sankar [94] hervor, dass die erhaltenen Ergebnisse auch für Werte $k<0$ verwendbar sind. Obwohl der Zusammenhang (3.209) wegen des an der Oberfläche wahlweise verschwindenden $(k>0)$ oder divergierenden $(k<0)$ Moduls quantitativ sicher problematisch ist, soll in dem vorliegenden Buch auf diesen zurückgegriffen werden, da für andere Formen der Inhomogenität bereits die Fundamentallösung, also die Grundlage aller kontaktmechanischen Rechnungen, nur numerisch bestimmbar ist. Außerdem lassen sich alle qualitativen Effekte der Gradierung auch durch den Zusammenhang (3.209) abbilden ${ }^{13}$. Mit $k=0$ steht als zusätzlicher Vorteil darüber hinaus jederzeit der homogene Grenzfall (mit den bekannten Lösungen) als Testlösung bereit.

Obwohl die Bestimmung der JKR-adhäsiven Lösung des axialsymmetrischen Normalkontaktproblems aus der nicht-adhäsiven Lösung auch für inhomogene Medien keine größere Schwierigkeit darstellt, existieren vollständige Lösungen von adhäsiven Normalkontaktproblemen mit FGM erst seit etwa 10 Jahren [95-97]. Tangentialkontakte von FGM sind sogar erst seit wenigen Jahren durch die Arbeit von Heß und Popov [98] einer analytischen Behandlung zugänglich. Andererseits können durch das viskoelastische Korrespondenz-

\footnotetext{
${ }^{13}$ Insofern gelten für dieses Modell ähnliche Dinge wie die, die an früherer Stelle über das DugdaleModell der adhäsiven Spannung gesagt wurden.
} 
prinzip unter bestimmten Umständen auch Kontaktprobleme von viskoelastischen inhomogenen Medien erfasst werden [99]. Dies ist besonders für biologische Gradientenmedien interessant, da diese in der Regel visko- oder poroelastische Eigenschaften aufweisen [84].

\subsubsection{Fundamentallösung des inhomogenen Halbraums}

Im Fall eines Halbraums mit einer elastischen Gradierung in der Form eines Potenzgesetzes wurde von Booker et al. [89] die Fundamentallösung für die Verschiebungen des Halbraums unter Einwirkung einer Punktlast im Ursprung hergeleitet. Für die Verschiebungen der Halbraumoberfläche ergibt sich

$$
\begin{aligned}
& u_{x}=\frac{z_{0}^{k}}{4 \pi G_{0} r^{3+k}}\left[\left(H x^{2}+P y^{2}\right) F_{x}+(H-P) x y F_{y}+A x r F_{z}\right], \\
& u_{y}=\frac{z_{0}^{k}}{4 \pi G_{0} r^{3+k}}\left[\left(H y^{2}+P x^{2}\right) F_{y}+(H-P) x y F_{x}+A y r F_{z}\right], \\
& u_{z}=\frac{z_{0}^{k}}{4 \pi G_{0} r^{3+k}}\left[\operatorname{Lr}\left(x F_{x}+y F_{y}\right)+B r^{2} F_{z}\right],
\end{aligned}
$$

mit den Kürzeln

$$
\begin{aligned}
& B:=\frac{2(1-v)}{(1+k)^{2}} \beta \sin \left(\frac{\pi \beta}{2}\right) \frac{\Gamma\left(\frac{3+k+\beta}{2}\right) \Gamma\left(\frac{3+k-\beta}{2}\right)}{\left[\Gamma\left(1+\frac{k}{2}\right)\right]^{2}}, \\
& L:=-\frac{2(1-v)}{k} \cos \left(\frac{\pi \beta}{2}\right) \frac{\Gamma\left(\frac{3+k+\beta}{2}\right) \Gamma\left(\frac{3+k-\beta}{2}\right)}{\Gamma\left(\frac{1+k}{2}\right) \Gamma\left(\frac{3+k}{2}\right)}=-A
\end{aligned}
$$

für die vertikale Verschiebung, wobei die Hilfsgröße $\beta$ durch

$$
\beta:=\sqrt{(1+k)\left(1-\frac{k v}{1-v}\right)} .
$$

definiert ist, sowie den Kürzeln

$$
\begin{aligned}
& H:=-\frac{2(1-v) k}{\beta(1-k)} \sin \left(\frac{\pi \beta}{2}\right) \frac{\Gamma\left(\frac{3+k+\beta}{2}\right) \Gamma\left(\frac{3+k-\beta}{2}\right)}{\left[\Gamma\left(1+\frac{k}{2}\right)\right]^{2}}+\frac{2}{1-k}, \\
& P:=\frac{2(1-v)}{\beta(1-k)} \sin \left(\frac{\pi \beta}{2}\right) \frac{\Gamma\left(\frac{3+k+\beta}{2}\right) \Gamma\left(\frac{3+k-\beta}{2}\right)}{\left[\Gamma\left(1+\frac{k}{2}\right)\right]^{2}}-\frac{2 k}{1-k}
\end{aligned}
$$

für die tangentiale Verschiebung. Im homogenen Fall $k=0$ erhält man die Lösungen von Boussinesq und Cerruti aus den Gl.(3.1) bis (3.3). 
Für den Kontakt zweier Körper (der Exponent $k$ der Gradierung muss für beide übereinstimmen), die der Halbraumnäherung genügen und die durch eine Punktkraft im gemeinsamen Koordinatenursprung aufeinander wirken, lauten die sich ergebenden relativen Verschiebungen damit wie folgt:

$$
\begin{aligned}
\Delta u_{x} & =\frac{F_{x}}{4 \pi r^{3+k}}\left[\left(\frac{z_{01}^{k}}{G_{01}} H_{1}+\frac{z_{02}^{k}}{G_{02}} H_{2}\right) x^{2}+\left(\frac{z_{01}^{k}}{G_{01}} P_{1}+\frac{z_{02}^{k}}{G_{02}} P_{2}\right) y^{2}\right] \\
& +\frac{x y F_{y}}{4 \pi r^{3+k}}\left(\frac{z_{01}^{k}}{G_{01}} H_{1}+\frac{z_{02}^{k}}{G_{02}} H_{2}-\frac{z_{01}^{k}}{G_{01}} P_{1}-\frac{z_{02}^{k}}{G_{02}} P_{2}\right)+\frac{x r F_{z}}{4 \pi r^{3+k}}\left(\frac{z_{01}^{k}}{G_{01}} A_{1}-\frac{z_{02}^{k}}{G_{02}} A_{2}\right), \\
\Delta u_{z} & =\frac{1}{4 \pi r^{2+k}}\left[\left(\frac{z_{01}^{k}}{G_{01}} L_{1}-\frac{z_{02}^{k}}{G_{02}} L_{2}\right)\left(x F_{x}+y F_{y}\right)+\left(\frac{z_{01}^{k}}{G_{01}} B_{1}+\frac{z_{02}^{k}}{G_{02}} B_{2}\right) r F_{z}\right] .
\end{aligned}
$$

Die beiden Körper sind elastisch ähnlich, d. h. die relativen tangentialen Verschiebungen durch die Normalbelastung und vice versa verschwinden, falls

$$
\frac{z_{01}^{k}}{G_{01}} L_{1}=\frac{z_{02}^{k}}{G_{02}} L_{2} .
$$

Insbesondere ist das der Fall bei gleichen Materialien, oder wenn ein Körper starr ist und der andere dem Holl-Verhältnis

$$
v=v_{H}:=\frac{1}{2+k}
$$

genügt, oder wenn beide Körper dem Holl-Verhältnis genügen. Es ist dabei offensichtlich, dass dieses Verhältnis nur für positive Exponenten $k$ physikalisch erfüllbar ist, da die thermodynamische Stabilität eines Mediums nur für $v \leq 0,5$ gegeben ist. Das bedeutet aber natürlich nicht, dass verschiedene Körper mit negativen Exponenten nicht elastisch ähnlich sein können, sie müssen nur die Bedingung (3.220) erfüllen.

Wenn ein Halbraum dem Holl-Verhältnis genügt, vereinfachen sich die oben eingeführten Kürzel bedeutend und man erhält

$$
\beta=1, \quad B=\frac{1}{1+k}, \quad L=0, \quad H=2+k, \quad P=1 .
$$

Falls die beteiligten Gradientenmedien elastisch ähnlich sind und der Kontakt der Halbraumhypothese genügt, kann der Kontakt zwischen zwei elastischen Körpern wie im homogenen Fall auf den Kontakt zwischen einem starren Indenter und einem elastischen Halbraum zurückgeführt werden. Die Verallgemeinerung der effektiven elastischen Moduln für Materialien mit einer elastischen Gradierung in der Form eines Potenzgesetzes sind die Moduln 


$$
\begin{aligned}
& c_{N}:=4 \cos \left(\frac{k \pi}{2}\right)\left(\frac{z_{01}^{k}}{G_{01}} B_{1}+\frac{z_{02}^{k}}{G_{02}} B_{2}\right)^{-1}, \\
& c_{T}:=8 \cos \left(\frac{k \pi}{2}\right)\left[\frac{z_{01}^{k}}{G_{01}}\left(H_{1}+P_{1}\right)+\frac{z_{02}^{k}}{G_{02}}\left(H_{2}+P_{2}\right)\right]^{-1} .
\end{aligned}
$$

Diese vereinfachen sich für $k=0 \mathrm{zu} \tilde{E}$ und $\tilde{G}$.

\subsubsection{Reibungsfreier Normalkontakt ohne Adhäsion}

Wie im elastisch homogenen Fall ist die Grundlage der allgemeinen axialsymmetrischen Lösung des reibungsfreien Normalkontaktproblems elastisch inhomogener Körper die Lösung des Problems der reibungsfreien Indentierung eines elastisch inhomogenen Halbraums durch einen starren flachen zylindrischen Stempel. Diese Lösung wurde ebenfalls von Booker et al. [90], basierend auf der von ihnen erhaltenen Fundamentallösung, vorgelegt. Die Normalkraft $F_{z}$ als Funktion der Eindrucktiefe $d$ und des Stempelradius $a$ beträgt

$$
F_{z}=-2 c_{N} \frac{d a^{1+k}}{1+k}
$$

woraus man die Kontaktsteifigkeit

$$
k_{z}=2 c_{N} \frac{a^{1+k}}{1+k}
$$

erhält. Die Spannungsverteilung im Kontakt hat die Form

$$
\sigma_{z z}(r)=-\frac{c_{N} d}{\pi \sqrt{\left(a^{2}-r^{2}\right)^{1-k}}}, \quad r \leq a,
$$

und die Verschiebungen außerhalb des Kontaktes können zu

$$
u_{z}(r)=-\frac{d}{\pi} \cos \left(\frac{k \pi}{2}\right) \mathrm{B}\left(\frac{a^{2}}{r^{2}} ; \frac{1+k}{2}, \frac{1-k}{2}\right), \quad r>a,
$$

bestimmt werden. Es sei darauf hingewiesen, dass diese Lösung (wie im homogenen Fall) - obwohl die Definition des Normalmoduls $c_{N}$ in Gl.(3.223) impliziert, dass beide Körper elastisch sein können - nur für den Kontakt mit einem starren Stempel gültig ist, da der elastische Stempel unbedingt die Annahmen der Halbraumhypothese verletzt.

Durch eine geeignete Superposition von infinitesimalen Stempellösungen kann man nun, in völliger Analogie zu dem in Abschn.3.2.2 ausführlich hergeleiteten homogenen Fall, die Lösung für eine beliebige axialsymmetrische Indenterform $f(r)$ bestimmen. Man erhält für 
die gesamte Normalkraft, die Spannungsverteilung und die Verschiebungen nach Abschluss des Indentierungsvorgangs [97]:

$$
\begin{aligned}
F_{z} & =-2 c_{N} \int_{0}^{a}[d-g(x)] x^{k} \mathrm{~d} x, \\
\sigma_{z z}(r) & =-\frac{c_{N}}{\pi} \int_{r}^{a} \frac{g^{\prime}(x) \mathrm{d} x}{\sqrt{\left(x^{2}-r^{2}\right)^{1-k}}}, \quad r \leq a, \\
u_{z}(r) & =-\frac{2}{\pi} \cos \left(\frac{k \pi}{2}\right) \int_{0}^{\min (r, a)} \frac{x^{k}[d-g(x)] \mathrm{d} x}{\sqrt{\left(r^{2}-x^{2}\right)^{1+k}}} .
\end{aligned}
$$

Die Verschiebungen innerhalb des Kontaktes sind durch den Eindruckkörper vorgegeben, also

$$
f(r)=\frac{2}{\pi} \cos \left(\frac{k \pi}{2}\right) \int_{0}^{r} \frac{x^{k} g(x) \mathrm{d} x}{\sqrt{\left(r^{2}-x^{2}\right)^{1+k}}} .
$$

Dies ist eine verallgemeinerte Abel-Transformation, die mit dem Ergebnis [97]

$$
g(x)=|x|^{1-k} \int_{0}^{|x|} \frac{f^{\prime}(r) \mathrm{d} r}{\sqrt{\left(x^{2}-r^{2}\right)^{1-k}}}
$$

invertiert werden kann. Damit ist das Kontaktproblem in allgemeiner Form gelöst. Für den parabolischen Kontakt mit dem Krümmungsradius $\tilde{R}$ in der Nähe des Kontaktes ergibt sich

$$
\begin{aligned}
g(x) & =\frac{x^{2}}{\tilde{R}(1+k)}, \\
d & =\frac{a^{2}}{\tilde{R}(1+k)}, \\
F_{z} & =-\frac{4 c_{N} a^{3+k}}{\tilde{R}(1+k)^{2}(3+k)}, \\
\sigma_{z z}(r) & =-\frac{2 c_{N}}{\pi \tilde{R}(1+k)^{2}} \sqrt{\left(a^{2}-r^{2}\right)^{1+k}}, \quad r \leq a,
\end{aligned}
$$

was im homogenen Fall $k=0$ natürlich mit der Hertzschen Lösung übereinstimmt.

Für ein Profil in der Form eines Potenzgesetzes (siehe Gl. (3.38)) lautet die Lösung des Kontaktproblems für die makroskopischen Größen wie folgt: 


$$
\begin{aligned}
d(a) & =\beta(n, k) A a^{n}, \quad \beta(n, k):=\frac{n}{2} \mathrm{~B}\left(\frac{n}{2}, \frac{1+k}{2}\right) \\
F_{z}(a) & =-\frac{2 n c_{N} \beta(n, k) A}{(1+k)(n+k+1)} a^{n+k+1},
\end{aligned}
$$

mit der im Anhang definierten vollständigen Beta-Funktion $\mathrm{B}(\cdot, \cdot)$.

Die entscheidende Größe für die dynamischen Eigenschaften eines Kontaktes ist die Kontaktsteifigkeit als Funktion der Eindrucktiefe $d$. Im Fall des Potenzprofils ergibt sich hierfür

$$
k_{z}(d)=\frac{2}{1+k} c_{N}\left(\frac{1}{\beta(n, k) A}\right)^{\frac{1+k}{n}} d^{\frac{1+k}{n}} .
$$

Man erkennt, dass sich derselbe Zusammenhang auch für den Kontakt eines Indenters mit einem homogenen elastischen Halbraum mit dem effektiven Modul $\tilde{E}$ ergibt, wenn man den Exponent $\tilde{n}$ des Indenterprofils entsprechend

$$
\tilde{n}:=\frac{n}{1+k}
$$

wählt. Die dynamischen Eigenschaften eines Kontaktes mit einem FGM sind also durch ein bestimmtes homogenes Problem exakt abbildbar. Die Konstante $\tilde{A}$ des äquivalenten homogenen Problems ${ }^{14}$ ergibt sich zu

$$
\tilde{A}=A\left(\frac{\tilde{E}}{c_{N}}\right)^{\tilde{n}}(1+k)^{\tilde{n}} \frac{\beta(n, k)}{\beta(\tilde{n}, 0)} .
$$

\subsubsection{Reibungsfreier Normalkontakt mit Adhäsion in der JKR-Näherung}

Die Tatsache, dass der JKR-adhäsive (reibungsfreie) Normalkontakt zwischen einem axialsymmetrischen starren Indenter und einem elastischen Halbraum aus einer Superposition des entsprechenden nicht-adhäsiven Problems mit einer Indentierung durch einen flachen zylindrischen Stempel hervorgeht, folgt unmittelbar aus der Gleichheit der gemischten Randbedingungen des nicht-adhäsiven und des JKR-adhäsiven Problems und ist daher unabhängig von den elastischen Eigenschaften des Halbraums. Insbesondere gilt dies auch für die spezielle Form der elastischen Inhomogenität, die in diesem Unterkapitel behandelt wird, der Gradierung in der Form eines Potenzgesetzes.

Die Lösung des nicht-adhäsiven Problems wurde oben gezeigt. Bei der Herleitung der JKR-adhäsiven aus der nicht-adhäsiven Lösung (siehe Abschn. 3.3.2) muss man in Gl. (3.54) nur den Ausdruck der (nicht-adhäsiven) inkrementellen Kontaktsteifigkeit aus Gl.(3.226)

\footnotetext{
${ }^{14}$ Die Äquivalenz erstreckt sich natürlich nicht über lokale Größen, wie z. B. Spannungen.
} 
einsetzen. Man erhält (wie im Abschn.3.3.2 bezeichnet das Superskript „n.a.“ die nichtadhäsiven Größen):

$$
\begin{aligned}
& d=d^{\text {n.a. }}-\Delta l=d^{\text {n.a. }}-\sqrt{\frac{2 \pi \Delta \gamma}{c_{N}} a^{1-k}} \\
& F_{z}=F_{z}^{\text {n.a. }}+k_{z}^{\text {n.a. }} \Delta l=F_{z}^{\text {n.a. }}+\frac{2}{1+k} \sqrt{2 \pi \Delta \gamma c_{N} a^{3+k}} .
\end{aligned}
$$

Für den parabolischen Kontakt ergibt sich entsprechend

$$
\begin{aligned}
d & =\frac{a^{2}}{\tilde{R}(1+k)}-\sqrt{\frac{2 \pi \Delta \gamma}{c_{N}} a^{1-k}}, \\
F_{z} & =-\frac{4 c_{N} a^{3+k}}{\tilde{R}(1+k)^{2}(3+k)}+\frac{2}{1+k} \sqrt{2 \pi \Delta \gamma c_{N} a^{3+k}}
\end{aligned}
$$

als Lösung des JKR-adhäsiven (reibungsfreien) Normalkontaktproblems. Der instantane Kontaktradius für $d=0$ beträgt

$$
a_{0}=\left[\frac{2 \pi \Delta \gamma \tilde{R}^{2}(1+k)^{2}}{c_{N}}\right]^{\frac{1}{3+k}}
$$

Die kritische Konfiguration, bei der der Kontakt seine Stabilität verliert und sich auflöst, ist im Fall der Kraftsteuerung durch die Beziehungen

$$
\begin{aligned}
d_{c}^{\mathrm{KS}} & =\frac{k-1}{(1+k)(3+k) \tilde{R}}\left[\frac{\pi(1+k)^{2}(3+k)^{2} R^{2} \Delta \gamma}{8 c_{N}}\right]^{\frac{2}{3+k}}, \\
a_{c}^{\mathrm{KS}} & =\left[\frac{\pi(1+k)^{2}(3+k)^{2} \tilde{R}^{2} \Delta \gamma}{8 c_{N}}\right]^{\frac{1}{3+k}}, \\
F_{z, c}^{\mathrm{KS}} & =\frac{3+k}{2} \pi \Delta \gamma \tilde{R}
\end{aligned}
$$

und im Fall der Wegsteuerung durch

$$
\begin{aligned}
& d_{c}^{\mathrm{WS}}=-\frac{3+k}{\left(1-k^{2}\right) R}\left[\frac{\pi\left(1-k^{2}\right)^{2} \tilde{R}^{2} \Delta \gamma}{8 c_{N}}\right]^{\frac{2}{3+k}}, \\
& a_{c}^{\mathrm{WS}}=\left[\frac{\pi\left(1-k^{2}\right)^{2} \tilde{R}^{2} \Delta \gamma}{8 c_{N}}\right]^{\frac{1}{3+k}}, \\
& F_{z, c}^{\mathrm{WS}}=\frac{(1-k)(3 k+5)}{2(3+k)} \pi \Delta \gamma \tilde{R}
\end{aligned}
$$


gegeben. Wie von Chen et al. [95] angemerkt wurde, ist die maximale Adhäsionskraft (bei Kraftsteuerung) im Fall des Gibson-Mediums, also des inkompressiblen linear-gradierten Halbraums ( $k \rightarrow 1, v=0,5$ ), gleich $2 \pi \Delta \gamma R$, d. h. gleich der maximalen Adhäsionskraft im DMT-Grenzfall der Adhäsion ${ }^{15}$. Die Erklärung dieses Phänomens - für steigende Werte des Exponenten $k$ verringert sich die Differenz zwischen dem DMT- und dem JKR-Verhalten wurde erst durch die kürzlich publizierte Lösung des Dugdale-Maugis-adhäsiven Normalkontaktproblems von axialsymmetrischen Körpern mit einer elastischen Gradierung in der Form eines Potenzgesetzes geliefert [100].

\subsubsection{Tangentialkontakt}

Analytische Lösungen von Tangentialkontaktproblemen elastisch gradierter Materialien existieren, wie gesagt, erst seit sehr kurzer Zeit. Der Aufbau des folgenden Abschnitts orientiert sich dabei an der Struktur des Unterkapitels 3.4, das dem Tangentialkontakt elastisch homogener Medien gewidmet ist; zunächst wird das Problem ohne lokales Gleiten (also mit einem unendlich großen Reibkoeffizienten) gelöst und anschließend der Einfluss endlicher Reibung berücksichtigt. Die Kontaktpartner seien einander grundsätzlich elastisch ähnlich.

\section{Kontakt ohne Gleiten}

Verschiebt man ein kreisförmiges Gebiet mit dem Radius $a$ an der Oberfläche eines Halbraums mit einer elastischen Gradierung in der Form (3.209) als Ganzes um $u_{x, 0}$, so ist dafür eine tangentiale $\mathrm{Kraft}^{16}$

$$
F_{x}=\frac{2}{1+k} c_{T} a^{1+k} u_{x, 0}
$$

mit dem in Gl.(3.224) gegebenen Tangentialmodul des Gradientenmediums, notwendig. Die Verteilung der Scherspannungen in der Kontaktfläche hat die Form

$$
\sigma_{x z}(r)=\frac{c_{T}}{\pi} \frac{u_{x, 0}}{\sqrt{\left(a^{2}-r^{2}\right)^{1-k}}}, \quad r \leq a,
$$

und die tangentiale Kontaktsteifigkeit des haftenden Kontaktes ergibt sich zu

$$
k_{x}=2 c_{T} \frac{a^{1+k}}{1+k} .
$$

Eine wichtige Größe ist der häufig „,Mindlin-Verhältnis“ genannte Quotient $l$ aus der tangentialen und normalen Kontaktsteifigkeit des Flachstempelkontaktes. Dieser ist unabhängig von der Kontaktkonfiguration durch das Verhältnis der Moduln

\footnotetext{
${ }^{15}$ Die maximale Adhäsionskraft in der DMT-Theorie entspricht einer Konfiguration ohne direkten Kontakt; sie ist daher grundsätzlich von den elastischen Eigenschaften unabhängig.

${ }^{16}$ Die Herleitung der Lösung dieses Kontaktproblems ist im Anhang gegeben.
} 
Abb.3.13 Konturlinien-

Diagramm für das

Mindlin-Verhältnis zwischen tangentialer und normaler Kontaktsteifigkeit für einen gradierten elastischen Halbraum als Funktion des Exponenten $k$ der elastischen Gradierung und der Poissonzahl $v$ (für den gesamten thermodynamisch stabilen Wertebereich von $v$ )

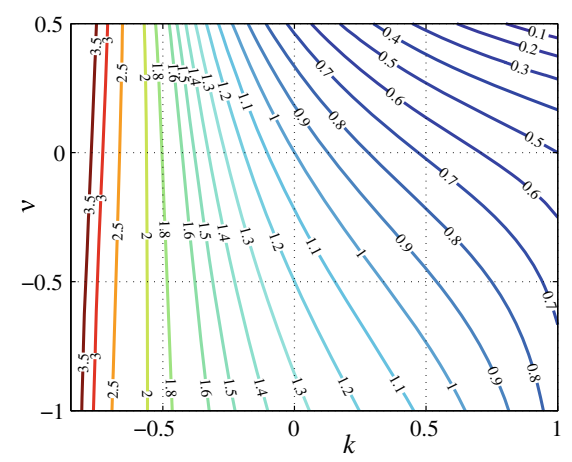

$$
l:=\frac{k_{x}}{k_{z}}=\frac{c_{T}}{c_{N}}
$$

gegeben. Wenn ein Medium starr ist und das zweite dem Holl-Verhältnis (3.221) genügt, oder beide Medien dem Holl-Verhältnis genügen, vereinfacht sich dieser Ausdruck zu [101]

$$
l=\frac{2}{(1+k)(3+k)} .
$$

Für ein einzelnes elastisches Medium mit einer Gradierung in der Form eines Potenzgesetzes hängt $l$ nur von dem Exponent $k$ und der Poissonzahl $v$ (und nicht von $G_{0}$ oder $z_{0}$ ) ab. Der Zusammenhang $l=l(k, v)$ ist in Abb. 3.13 als Konturlinien-Diagramm dargestellt.

\section{Kontakt mit Gleiten}

Eine axialsymmetrische Spannungsverteilung der Form

$$
\sigma_{x z}(r)=\sigma_{1}\left(1-\frac{r^{2}}{a^{2}}\right)^{\frac{1+k}{2}}, \quad r \leq a,
$$

erzeugt an der Oberfläche eines Mediums mit einer elastischen Gradierung in der Form eines Potenzgesetzes mit dem Exponenten $k$ innerhalb des Kontaktgebiets die tangentialen Verschiebungen $^{17}$

$$
\begin{aligned}
& u_{x}(x, y)=\frac{\pi \sigma_{1}(1+k)}{2 c_{T}} a^{1-k}\left\{1-\left[1-\frac{(1-k)(3 H+P)}{4(H+P)}\right] \frac{x^{2}}{a^{2}}-\left[1-\frac{(1-k)(H+3 P)}{4(H+P)}\right] \frac{y^{2}}{a^{2}}\right\}, \\
& u_{y}(x, y)=\frac{\pi \sigma_{1} z_{0}^{k}\left(1-k^{2}\right)(H-P) x y}{4 c_{T}(H+P) a^{1+k}}
\end{aligned}
$$

\footnotetext{
${ }^{17}$ Die Lösung dieses Randwertproblems mithilfe der Fundamentallösung des Gradientenmaterials ist im Anhang gegeben.
} 
mit den in den Gl.(3.216) und (3.217) eingeführten Kürzeln $H$ und $P$. Auf die Angabe der Verschiebungen außerhalb des Kontaktgebiets wurde verzichtet, da das tangentiale Kontaktproblem mit Gleiten, wie im homogenen Fall, im Rahmen der Cattaneo-MindlinNäherung (Vernachlässigung der Querverschiebungen im Gleitgebiet, Gültigkeit eines lokalen Amontons-Coulomb-Reibgesetzes) gelöst werden soll. Allerdings existieren für Gradientenmedien - im Gegensatz zu homogenen Medien - noch keine publizierten Studien zu dem aus diesen vereinfachenden Annahmen resultierenden Fehler.

Im Rahmen dieser Vereinfachung ist mit den oben angegebenen Verschiebungen und der Lösung des Normalkontaktproblems klar, dass eine Spannungsverteilung der Form

$$
\sigma_{x z}(r)=\frac{2 \mu c_{N}}{\pi \tilde{R}(1+k)^{2}} \begin{cases}\left(a^{2}-r^{2}\right)^{\frac{1+k}{2}}-\left(c^{2}-r^{2}\right)^{\frac{1+k}{2}}, & r \leq c, \\ \left(a^{2}-r^{2}\right)^{\frac{1+k}{2}}, & c<r \leq a,\end{cases}
$$

mit dem Haftradius $c$, die gemischten Randbedingungen des Tangentialkontaktproblems von Kugeln erfüllt. Das Ciavarella-Jäger-Theorem gilt also im Rahmen der Cattaneo-MindlinNäherung auch für den Tangentialkontakt von Kugeln mit der beschriebenen elastischen Gradierung. Die tangentiale Starrkörperverschiebung und die gesamte Tangentialkraft bestimmt man leicht $\mathrm{zu}$

$$
\begin{aligned}
u_{x, 0} & =\frac{\mu c_{N}}{c_{T}}\left(\frac{a^{2}}{\tilde{R}(1+k)}-\frac{c^{2}}{\tilde{R}(1+k)}\right), \\
F_{x} & =-\mu F_{z}\left[1-\left(\frac{c}{a}\right)^{3+k}\right] .
\end{aligned}
$$

Da die Spannungsverteilung (3.262) die Superpositionsregel (3.109) erfüllt, ist der in Abschn.3.4.3 geschilderte Formalismus zur Behandlung beliebiger Belastungsgeschichten für den axialsymmetrischen Tangentialkontakt homogener Medien auch für das hier untersuchte, elastisch inhomogene Tangentialkontaktproblem anwendbar.

\subsection{Plastizität}

\subsubsection{Einführung}

Die Spannungen in mechanischen Kontakten, gerade bei stoßartiger Belastung, sind oft so hoch, dass es zumindest lokal zu plastischer Deformation kommt. Die Berücksichtigung der Plastizität für das Kontaktproblem ist in analytischer Form äußerst kompliziert, da zu diesem Zweck in der Regel die Kenntnis des vollständigen Spannungszustands auch innerhalb der kontaktierenden Körper notwendig ist. Das liegt daran, dass das Maximum der Vergleichsspannung (und damit der Ausgangspunkt der plastischen Deformation) sehr häufig unterhalb der Oberfläche lokalisiert ist. Es ist dabei bemerkenswert, dass der Spannungszustand direkt unterhalb eines nur in normaler Richtung belasteten Kontaktes zum Großteil reiner 
hydrostatischer Kompression entspricht [11, S. 173 f.]. In elasto-plastischen Kontakten gibt es deswegen in der Regel einen elastisch deformierten „Kern“ in der unmittelbaren Umgebung des Kontaktes, der von einem plastisch deformierten Gebiet eingeschlossen ist.

Die Phänomenologie von elasto-plastischen Kontaktproblemen kann man grob in vier Stadien unterteilen: Bei ausreichend kleinen Belastungen sind alle Deformationen elastisch; bei größeren Lasten folgt ein elasto-plastischer Bereich, der schließlich in ein voll-plastisches Stadium mit unbeschränktem Fließen übergeht. Die Entlastung ist ein im Wesentlichen elastischer Prozess [11, S. 181 ff.], [102] mit einem durch die vorherige plastische Verformung veränderten Profil.

Einen hervorragenden und aktuellen Überblick über die verschiedenen theoretischen Ansätze in diesem Bereich (einschließlich der Behandlung von Adhäsion, Reibung und Rauigkeit) bietet die Publikation von Ghaednia et al. [103]; deswegen sollen an dieser Stelle nur die wichtigsten Arbeiten zu dem Thema kurz beschrieben werden.

\subsubsection{Normalkontakt ohne Adhäsion (parabolischer Kontakt)}

Die ersten Publikationen zu plastischen Kontaktproblemen (und ein Großteil der experimentellen Arbeiten auf dem Gebiet) stammen aus dem Bereich der Härtemessung. Der Indentierung eines elasto-plastischen Mediums (Halbraums) durch eine starre Kugel entspricht dabei das Brinell-Verfahren. Dieses Problem wurde für den starr-plastischen Fall von Ishlinski [104] mithilfe des Gleitlinienverfahrens vollständig analytisch gelöst.

Ein Klassiker auf dem Gebiet der Härtemessung ist das Buch von Tabor [105]. Er begründete, dass die Spannungsverteilung im voll-plastischen Kontakt näherungsweise konstant ist und diese konstante Spannung (d.h. die Härte) in etwa dem Dreifachen der Fließgrenze entspricht [105, S. 17].

Seit knapp 40 Jahren bedienen sich die meisten theoretischen, der Problematik gewidmeten Arbeiten numerischer Lösungen mithilfe der Finite-Elemente-Methode (FEM). Durch die Fortschritte in der Entwicklung der Methode und der Rechenleistung moderner Computer waren dabei FEM-basierte Modelle von elasto-plastischen Normalkontakten in der Lage, immer mehr Effekte immer besser zu berücksichtigen und zu untersuchen, beginnend mit der elastisch-ideal-plastischen Indentierung durch eine starre Kugel [106], über die Berücksichtigung der Verfestigung [107-109] und des Reibregimes [110, 111] bis zur Betrachtung des Kontaktes zweier elasto-plastischer Körper [112]. Anders als für elastische Kontakte (im Rahmen der Halbraumnäherung) wurden dabei, besonders bei großen plastischen Deformationen, Unterschiede zwischen der Indentierung (also dem Kontakt eines starren gekrümmten Körpers mit einem elasto-plastischen Halbraum) und der Verflachung (d. h. dem Kontakt eines elasto-plastischen Körpers mit einer starren Ebene) beobachtet [113, 114]. Diese Unterschiede sind im Wesentlichen darauf zurückzuführen, dass zur Erzeugung starker plastischer Deformation Eindrucktiefen nötig sind, die die Halbraumannahme grob 
verletzen. Die makroskopische Form des deformierten Körpers spielt dann eine Rolle, beispielsweise für das Fließverhalten.

Da FEM-Modelle von dynamischen Problemen immer noch sehr rechenintensiv sind, wurden seit etwa 20 Jahren auch wieder verstärkt analytische Näherungslösungen für das elasto-plastische Normalkontaktproblem gesucht - meist mit Bezug auf das jeweilige Stoßproblem und daher fokussiert auf den Zusammenhang zwischen Normalkraft und Eindrucktiefe. Vu-Quoc et al. [115] schlugen ein einfaches Modell vor, das sie mithilfe von FEM-Rechnungen validierten, beziehungsweise anpassten. Leider enthält dieses Modell zwei empirische Parameter, die nicht a priori bekannt sind und für jedes Kontaktproblem separat aus FEM-Modellen oder Experimenten bestimmt werden müssen. Ein analytisches Modell ohne zusätzliche fit-Parameter stammt von Thornton [116]. Ein weiterer analytischer Ansatz, der von Zhao et al. [117] und Brake [118] verfolgt wurde, besteht in der Interpolation im elasto-plastischen Bereich zwischen den analytischen Lösungen für den elastischen und voll-plastischen Bereich. Da beide Modelle, der Ansatz von Thornton und die elasto-plastische Interpolation, im weiteren Verlauf dieses Buches zur Behandlung des elasto-plastischen Normalstoßes herangezogen werden, sind sie im Folgenden detaillierter ausgeführt. Eine weitere Möglichkeit der analytischen Behandlung und daher ebenfalls dargestellt sind außerdem analytische Approximationen von rigorosen FEM-Lösungen.

\section{Das Modell von Thornton}

Das Maximum der Vergleichsspannung nach von-Mises im Hertzschen Kontakt liegt unterhalb der Oberfläche; dieses Maximum kann man mit der Lösung von Huber [119] für die Spannungen im Inneren des Halbraums bestimmen. Johnson [11, S. 155] gab für den Hertzschen Normalkontakt an, dass lokales Fließen (sowohl nach dem Kriterium von Tresca als auch dem nach von-Mises) im Inneren bei einem Druck

$$
p_{\max }=1,6 \sigma_{f}
$$

mit der Fließgrenze $\sigma_{f}$ in einem einachsigen Zugversuch ${ }^{18}$, beginnt. Die entsprechenden kritischen Werte der Anpresskraft, des Kontaktradius und der Indentierungstiefe sind

$$
F_{Y}:=\frac{4}{3}\left(\frac{4 \pi}{5}\right)^{3}\left(\frac{\sigma_{f}}{\tilde{E}}\right)^{3} \tilde{E} \tilde{R}^{2}, \quad a_{Y}:=\frac{4 \pi}{5} \frac{\sigma_{f}}{\tilde{E}} \tilde{R}, \quad d_{Y}:=\left(\frac{4 \pi}{5}\right)^{2}\left(\frac{\sigma_{f}}{\tilde{E}}\right)^{2} \tilde{R} .
$$

Die Idee von Thornton besteht nun darin, von der elastischen Druckverteilung auszugehen und diese bei dem kritischen Wert $p_{Y}:=1,6 \sigma_{f}$,,abzuschneiden“. Für den Hertzschen Kontakt ergibt sich für die Druckverteilung im elasto-plastischen Bereich beispielsweise der Zusammenhang

\footnotetext{
${ }^{18}$ Wenn beide Körper deformierbar sind, bezeichnet $\sigma_{f}$ die Fließgrenze des weniger festen Mediums. Das gleiche gilt für die Härte $\sigma_{0}$.
} 


$$
\sigma_{z z}(r)=-\frac{2 \tilde{E}}{\pi \tilde{R}}\left\{\begin{array}{lr}
\sqrt{a^{2}-r^{2}}, & a_{p}<r \leq a, \\
\sqrt{a^{2}-a_{p}^{2}}, & r \leq a_{p},
\end{array}\right.
$$

mit

$$
p_{Y}=\frac{2 \tilde{E} a_{Y}}{\pi \tilde{R}}=\frac{2 \tilde{E}}{\pi \tilde{R}} \sqrt{a^{2}-a_{p}^{2}},
$$

der in Abb. 3.14 schematisch gezeigt ist. Aus der letzten Gl.(3.268) folgt, dass

$$
a^{2}=a_{Y}^{2}+a_{p}^{2}
$$

Die gesamte Normalkraft wird durch Integration der Druckverteilung bestimmt und mit Gl. (3.269) erhält man

$$
F_{z}=2 \pi \int_{0}^{a} \sigma_{z z}(r) r \mathrm{~d} r=-\frac{8 \pi}{15} \sigma_{f}\left(3 a^{2}-a_{Y}^{2}\right) .
$$

Wenn man außerdem annimmt, dass der Zusammenhang zwischen Kontaktradius und Eindrucktiefe (zumindest für nicht zu starke plastische Deformation) weiterhin durch die elastische Lösung $a^{2}=d \tilde{R}$ gegeben ist, lautet der Zusammenhang zwischen Normalkraft und Eindrucktiefe im elasto-plastischen Bereich

$$
F_{z}(d)=-\frac{8 \pi}{15} \sigma_{f} \tilde{R}\left(3 d-d_{Y}\right)
$$

Diese (bemerkenswerterweise lineare) Gleichung beschreibt die Tangente an die elastische Hertzsche Lösung am Ende des elastischen Bereichs.

Die Entlastung (nach Erreichen der maximalen Eindrucktiefe $d_{\max }$ ) des Kontaktes ist ein weitgehend elastischer Prozess. Thornton nahm daher an, dass der Zusammenhang zwischen Normalkraft und Eindrucktiefe in der Entlastungsphase durch die elastische Lösung

Abb.3.14 Druckverteilung für den elastisch-ideal-plastischen Hertzschen Kontakt nach dem Modell von Thornton. Die dünnen Linien beschreiben elastische Druckverteilungen nach Hertz

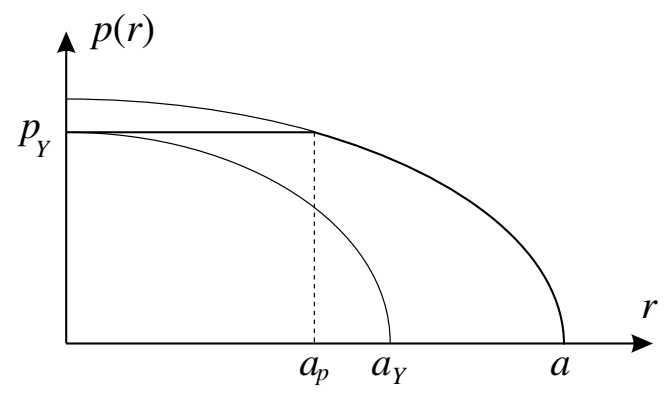




$$
F_{z}=-\frac{4}{3} \tilde{E} \sqrt{R_{p}\left(d-d_{\text {res }}\right)^{3}}
$$

mit dem durch die plastische Deformation vergrößerten Krümmungsradius des Profils [11, S. 182]

$$
R_{p}=\frac{4 \tilde{E} a_{\max }^{3}}{3\left|F_{z, \max }\right|}
$$

beschrieben werden kann. Die nach der vollständigen Entlastung verbleibende Resteindrucktiefe ergibt sich aus der Stetigkeit des Kraftverlaufs zu

$$
d_{\mathrm{res}}=d_{\max }-\left(\frac{3\left|F_{z, \max }\right|}{4 \tilde{E} \sqrt{R_{p}}}\right)^{2 / 3}=d_{\max }-\frac{a_{\max }^{2}}{R_{p}} .
$$

Normiert man alle Größen auf die kritischen Werte zu Beginn des lokalen Fließens, kann Gl. (3.271) in dimensionsloser Form als

$$
\frac{F_{z}}{F_{Y}}=-\frac{1}{2}\left(3 \frac{d}{d_{Y}}-1\right)
$$

geschrieben werden. Für die Entlastungsphase ergibt sich

$$
\frac{F_{z}}{F_{Y}}=-\sqrt{\frac{R_{p}}{\tilde{R}}}\left[\frac{d}{d_{Y}}-\frac{d_{\max }}{d_{Y}}\left(1-\frac{\tilde{R}}{R_{p}}\right)\right]^{3 / 2},
$$

wobei wegen der Gl.(3.271) und (3.273) der Zusammenhang zwischen der maximalen Eindrucktiefe und dem Krümmungsradius während der Entlastung durch

$$
\frac{\tilde{R}}{R_{p}}=\frac{1}{2}\left(3-\frac{d_{Y}}{d_{\max }}\right) \sqrt{\frac{d_{Y}}{d_{\max }}} .
$$

gegeben ist. Offenbar hängt das Verhalten in diesem Modell nur von dem Verhältnis $d_{Y} / d_{\max }$ ab. In Abb. 3.15 sind die Verläufe der Normalkraft als Funktion der Eindrucktiefe in normierten Größen bei einem vollständigen Belastungs- und Entlastungszyklus für verschiedene Werte dieses Verhältnisses dargestellt.

Der hysteretische Energie-Verlust $\Delta U$ während eines vollständigen Lastzyklus kann ohne Schwierigkeiten durch Integration bestimmt werden. In normierten Größen erhält man den Zusammenhang

$$
\frac{|\Delta U|}{F_{Y} d_{Y}}=\frac{2}{5}+\frac{1}{4}\left(\frac{3 d_{\mathrm{max}}^{2}}{d_{Y}^{2}}-2 \frac{d_{\mathrm{max}}}{d_{Y}}-1\right)-\frac{2}{5} \sqrt{\frac{R_{p}}{\tilde{R}}}\left(\frac{d_{\mathrm{max}}}{d_{Y}}-\frac{d_{\mathrm{res}}}{d_{Y}}\right)^{5 / 2},
$$

der in Abb. 3.16 grafisch dargestellt ist. 
Abb.3.15 Normalkraft als Funktion der Eindrucktiefe für verschiedene maximale Eindrucktiefen nach dem Thornton-Modell. Die dünne Linie beschreibt die elastische Lösung

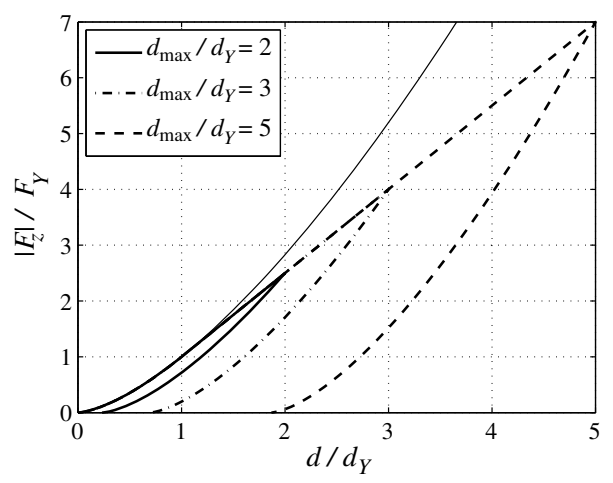

Abb.3.16 Normierter Hysterese-Verlust bei einem vollständigen Lastzyklus als Funktion der maximalen Eindrucktiefe nach dem Thornton-Modell

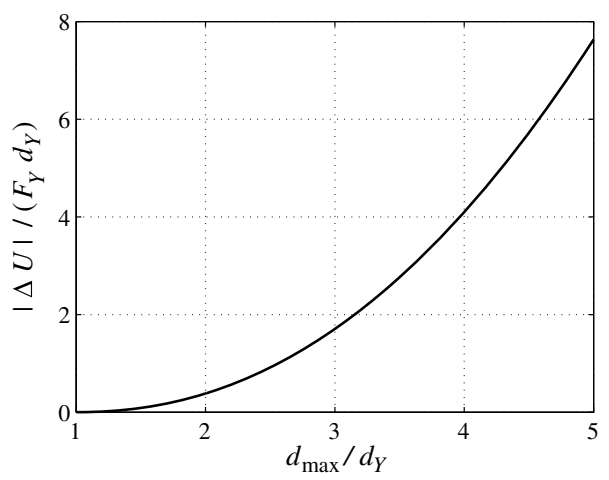

Die Lösung von Thornton ist nur am Anfang des elasto-plastischen Bereichs verwendbar, wenn der Kontakt noch weit entfernt von der voll-plastischen Konfiguration ist. Dies ist bereits daran erkennbar, dass für den Zusammenhang zwischen Kontaktradius und Eindrucktiefe weiterhin die elastische Lösung verwendet wird und der Maximaldruck im Kontakt im elasto-plastischen Bereich nicht weiter steigt. Diese beiden Schwächen des Modells wurden in einer modifizierten Version von Li et al. [120] behoben, allerdings auf Kosten deutlich reduzierter Handhabbarkeit und der Einführung eines fit-Parameters sowie weiterer, theoretisch schwer begründbarer Annahmen. Im Folgenden wird daher eine einfachere analytische Näherungslösung für den elasto-plastischen Kontakt geschildert, die man bis in den voll-plastischen Bereich verwenden kann.

\section{Interpolation im elasto-plastischen Bereich}

Bei rein elastischer Deformation der Kugeln ist der Zusammenhang zwischen Normalkraft und Eindrucktiefe durch die Hertzsche Lösung gegeben. In dimensionsloser Form lässt sich diese als 


$$
\frac{F_{z}^{\mathrm{el}}}{F_{Y}}=-\left(\frac{d}{d_{Y}}\right)^{3 / 2}, \quad d \leq d_{Y}
$$

schreiben. Für den voll-plastischen Bereich kann man unter einigen vereinfachenden Annahmen ebenfalls eine analytische Lösung konstruieren. Zunächst ist die Druckverteilung im voll-plastischen Kontakt, wie Experimente und FEM-Simulationen zeigen, in sehr guter Näherung konstant. Die gesamte Normalkraft ist daher einfach

$$
F_{z}^{\mathrm{pl}}=-\pi \sigma_{0} a^{2}
$$

mit der Härte $\sigma_{0}$. Der Zusammenhang zwischen Kontaktradius und Eindrucktiefe ist im voll-plastischen Bereich, wenn die Profile der Kugeln immer noch durch die parabolische Näherung beschreibbar sind,

$$
a=\sqrt{2 \tilde{R} d}+C
$$

Die Konstante $C$ hängt von dem genauen Fließverhalten ab und kann nur schwer theoretisch bestimmt werden. Nimmt man an, dass am Rand des Kontaktes durch die plastische Deformation Material weder aufgehäuft noch entfernt wird, ist der Wert von $C$ Null. In normierten Größen lautet die Beziehung zwischen Normalkraft und Eindrucktiefe in diesem Fall wie folgt:

$$
\frac{F_{z}^{\mathrm{pl}}}{F_{Y}}=-\frac{15 \sigma_{0}}{8 \sigma_{f}} \frac{d}{d_{Y}}:=-Q \frac{d}{d_{Y}}, \quad d>D d_{y} .
$$

Für metallische Werkstoffe entspricht die Härte ungefähr dem Dreifachen der Fließgrenze, die Konstante $Q$ hat also in etwa den Wert $Q \approx 5$. In mehreren Publikationen ${ }^{19}$ wurde allerdings darauf hingewiesen, dass die Härte mit dem Verhältnis $a / \tilde{R}$ fällt, beispielsweise nach dem Zusammenhang [121]

$$
\frac{\sigma_{0}}{\sigma_{Y}}=2,84-0,92\left[1-\cos \left(\frac{\pi a}{\tilde{R}}\right)\right] .
$$

Die „Konstante“ $Q$ ist also selbst schwach von der Eindrucktiefe abhängig. Da es sich bei dem Interpolationsmodell aber generell um ein recht grobes Verfahren handelt, soll diese Abhängigkeit im Rahmen der Präzision des Modells vernachlässigt werden. Der Wert der Konstante $D$ für den Gültigkeitsbereich der voll-plastischen Lösung ist ebenfalls nur schwer theoretisch begründbar; Johnson [11, S. 180] gab aufgrund experimenteller Ergebnisse den ungefähren Wert $D \approx 80$ an.

Für den elasto-plastischen Bereich kann man nun eine analytische Näherungslösung finden, indem zwischen der elastischen und der voll-plastischen Lösung stetig differenzierbar interpoliert wird. Obwohl dieses Verfahren im elasto-plastischen Bereich keine konkrete

${ }^{19}$ siehe die Übersicht von Ghaednia et al. [103] 
Physik beschreibt, bietet es in vielen Fällen, wie sich zeigen wird, eine gute Approximation des tatsächlichen Verhaltens. Zur Erfüllung aller Übergangsbedingungen ist für die Interpolation ein Polynom dritter Ordnung

$$
-\frac{F_{z}}{F_{Y}}=1+\frac{3}{2}\left(\frac{d}{d_{Y}}-1\right)+C_{2}\left(\frac{d}{d_{Y}}-1\right)^{2}+C_{3}\left(\frac{d}{d_{Y}}-1\right)^{3}, \quad d_{Y}<d \leq D d_{Y},
$$

hinreichend. Die Stetigkeit der Normalkraft und der Kontaktsteifigkeit am Übergang zum voll-plastischen Bereich liefert die Werte der noch unbestimmten Konstanten:

$$
C_{2}=\frac{Q(2 D+1)-3 D}{(D-1)^{2}}, \quad C_{3}=\frac{3 D+1-2 Q(D+1)}{2(D-1)^{3}} .
$$

Für den Kontaktradius als Funktion der Eindrucktiefe kann man ähnlich interpolieren. Die normierten Lösungen im elastischen und voll-plastischen Bereich sind

$$
\frac{a_{\mathrm{el}}^{2}}{a_{Y}^{2}}=\frac{d}{d_{Y}}, \quad \frac{a_{\mathrm{pl}}^{2}}{a_{Y}^{2}}=2 \frac{d}{d_{Y}} .
$$

Durch stetig differenzierbare Interpolation erhält man für den elasto-plastischen Bereich

$$
\frac{a^{2}}{a_{Y}^{2}}=1+\left(\frac{d}{d_{Y}}-1\right)+\frac{2 D+1}{(D-1)^{2}}\left(\frac{d}{d_{Y}}-1\right)^{2}-\frac{D+1}{(D-1)^{3}}\left(\frac{d}{d_{Y}}-1\right)^{3}, \quad d_{y}<d \leq D d_{Y} .
$$

Die elastische Entlastung kann man wiederum mithilfe der Gl. (3.272) bis (3.274) behandeln.

Der normierte Energieverlust durch die Hysterese beträgt für den Fall, dass nicht in den voll-plastischen Bereich belastet wird (d.h. $d_{\max } \leq D d_{Y}$ )

$$
\begin{aligned}
\frac{|\Delta U|}{F_{Y} d_{Y}} & =\frac{2}{5}+\left(\frac{d_{\max }}{d_{Y}}-1\right)+\frac{3}{4}\left(\frac{d_{\max }}{d_{Y}}-1\right)^{2}+\frac{C_{2}}{3}\left(\frac{d_{\max }}{d_{Y}}-1\right)^{3}+\frac{C_{3}}{4}\left(\frac{d_{\max }}{d_{Y}}-1\right)^{4} \\
& -\frac{2}{5} \sqrt{\frac{R_{p}}{\tilde{R}}}\left(\frac{d_{\max }}{d_{Y}}-\frac{d_{\mathrm{res}}}{d_{Y}}\right)^{5 / 2} .
\end{aligned}
$$

Die Bestimmung der Hysterese für den Fall $d_{\max }>D d_{Y}$ ist elementar aber länglich und soll daher an dieser Stelle aus Platzgründen ausgelassen werden.

Die Abbildungen 3.17 und 3.18 zeigen den normierten Normalkraftverlauf und den hysteretischen Energieverlust nach dem Interpolationsmodell für $Q=5$ und $D=80$. Vergleicht man mit den obigen Abbildungen der gleichen Zusammenhänge für das Modell von Thornton, erkennt man, dass letzteres eine deutlich geringere Kontaktsteifigkeit für den elasto-plastischen Bereich vorhersagt. Daraus resultiert im Thornton-Modell eine größere 
Abb.3.17 Verläufe aus Abb. 3.15 für das Interpolationsmodell mit $Q=5$ und $D=80$

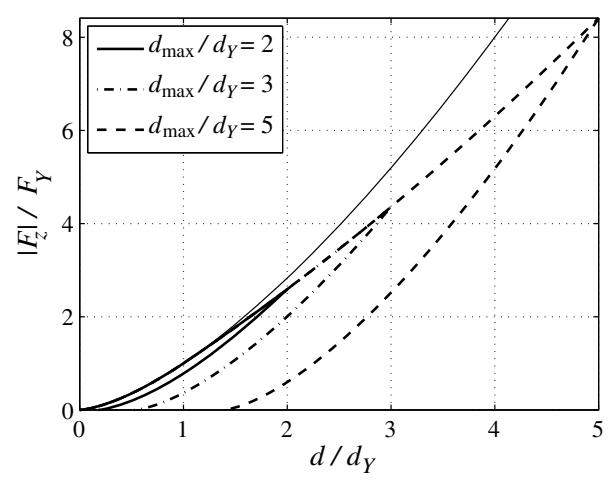

Abb. 3.18 Verlauf aus Abb. 3.16 für das Interpolationsmodell mit $Q=5$ und $D=80$

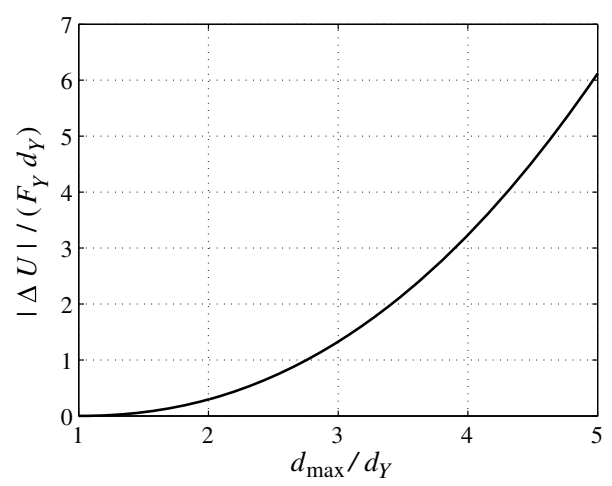

Resteindrucktiefe nach der Indentierung und ein größerer Energieverlust durch die elastoplastische Hysterese.

Es sei angemerkt, dass in der Wahl der voll-plastischen Lösung und der Interpolationsfunktionen eine gewisse Freiheit besteht; Brake [118] verwendete beispielsweise HermitePolynome und einen anderen Wert der Konstante $C$. Im obigen Text wurde dabei nur die, aus der Sicht des Autors, einfachste Variante dargestellt.

\section{Analytische Approximation von FEM-Lösungen}

Die korrekteste Variante zur Beschreibung der Lösung des elasto-plastischen Normalkontaktproblems mithilfe geschlossen analytischer Ausdrücke besteht sicherlich in der analytischen Approximation von rigorosen FEM-Rechnungen. $\mathrm{Zu}$ diesem Thema gibt es eine umfangreiche Literatur, an dieser Stelle soll aber nur ein einzelnes Modell gezeigt werden, das Jackson et al. [121] vorschlugen. Die Vorteile dieses Modells (im Rahmen des vorliegenden Buches) bestehen darin, dass es explizit für die Behandlung des Stoßproblems eingeführt und durch entsprechende Experimente validiert wurde. 
Jackson et al. untersuchten den Normalkontakt zwischen einer elasto-plastischen Kugel und einem ebenfalls elasto-plastischen Halbraum. Das weniger feste Medium ist der Halbraum. Dabei kann man praktisch von einem reinen Indentierungsproblem ausgehen, wenn die Zugfestigkeit der Kugel die des Halbraums um den Faktor 1,7 übersteigt [112]. Man unterscheidet wiederum drei Regime: den elastischen Bereich, der durch die Hertzsche Lösung beschrieben wird, den elasto-plastischen Bereich und die elastische Entlastung. Die elasto-plastische Lösung verwendeten Jackson et al. erst für Eindrucktiefen $d>1,9 d_{Y}^{*}$, mit dem modifizierten Ausdruck für die Eindrucktiefe, bei der die erste plastische Deformation einsetzt,

$$
d_{Y}^{*}:=\left(\frac{\pi C \sigma_{Y}}{2 \tilde{E}}\right)^{2} \tilde{R}, \quad C:=1,295 \exp (0,736 v),
$$

mit der Poissonzahl $v$ des Halbraums. Offenbar hängt diese Eindrucktiefe - zu der natürlich auch eine sich aus der Hertzschen Lösung ergebende Normalkraft $F_{Y}^{*}$ gehört - von $v$ ab, für $v=0,3$ stimmt sie allerdings fast genau mit dem Wert aus Gl. (3.266) überein. Die elasto-plastische Lösung lautet unter Verwendung des Kürzels $\delta:=d / d_{Y}^{*}$ wie folgt:

$$
-\frac{F_{z}}{F_{Y}^{*}}=\exp \left(-0,25 \delta^{5 / 12}\right) \delta^{3 / 2}+\frac{4 \sigma_{0}}{C \sigma_{Y}}\left[1-\exp \left(-\frac{1}{25} \delta^{5 / 9}\right)\right] \delta .
$$

Das Verhältnis zwischen Härte und Fließgrenze ist durch Gl. (3.283) bestimmt und der Kontaktradius im elasto-plastischen Regime ist durch die Beziehung

$$
a=\sqrt{\tilde{R} d}\left(\frac{\delta}{1,9}\right)^{B}, \quad B:=0,07 \exp \left(\frac{23 \sigma_{Y}}{\tilde{E}}\right)
$$

gegeben. Die Entlastung gehorcht wiederum der Gl. (3.272), wobei Jackson et al. für die Resteindrucktiefe den Ausdruck

$$
d_{\mathrm{res}}=1,02 d_{\max }\left[1-\left(\frac{d_{\max }+5,9 d_{Y}}{6,9 d_{Y}}\right)^{-0,54}\right]
$$

angaben. Der Krümmungsradius $R_{p}$ ergibt sich aus der Stetigkeit des Kraftverlaufs bei der maximalen Belastung.

\subsubsection{Normalkontakt mit Adhäsion (parabolischer Kontakt)}

Der adhäsive Normalkontakt elasto-plastischer Körper ist noch weit von einer umfassenden, robusten Beschreibung entfernt. Einzelne Aspekte des Problems wurden allerdings in der Literatur bereits behandelt.

Mesarovic und Johnson [122] untersuchten den Einfluss der Plastizität auf das Ablöseverhalten von adhäsiven Kugeln. Dazu nahmen sie an, dass man den Einfluss der Adhäsion 
während der elasto-plastischen Belastung vernachlässigen kann, und dass die Entlastung, wie im nicht-adhäsiven Fall, ein elastischer Prozess ist. Mit dem durch die plastische Deformation veränderten Profil am Ende der Belastung lösten sie das elastische Problem mit Adhäsion während der Abzugsphase im Rahmen der JKR-Theorie und der Theorie von Maugis und charakterisierten verschiedene Parameterbereiche mit unterschiedlichen Ablösemechanismen.

Ein weiterer wichtiger Beitrag stammt von Wu und Adams [123]. Diese bestimmten unter Verwendung des Adhäsionsmodells von Greenwood und Johnson [34] durch Betrachtung des vollständigen Spannungszustands im Inneren des elastischen Mediums die kritische Last, um unter einem adhäsiven Hertzschen Kontakt plastische Deformation zu initiieren, und stellten fest, dass diese kritische Last durch die Wirkung der Adhäsion deutlich herabgesetzt wird; dabei kann es in einem adhäsiven Kontakt sogar ohne eine äußere Normalkraft zu lokalem Fließen kommen. Abb. 3.19 zeigt die von Wu und Adams angegebene normierte kritische Normalkraft, um auf der Symmetrie-Achse lokales Fließen zu initiieren, als Funktion des Parameters

$$
\Phi:=\frac{\tilde{E}}{\pi \sigma_{f}}\left(\frac{16 \pi \Delta \gamma}{3 \tilde{R} \tilde{E}}\right)^{1 / 3}
$$

für verschiedene Poissonzahlen. Der Faktor $\Phi^{3}$ in der Normierung der Normalkraft stammt, bis auf einen numerischen Faktor, aus dem Verhältnis zwischen $3 \pi \Delta \gamma \tilde{R}$ und der in Gl. (3.266) gegebenen Fließgrenze $F_{Y}$ im nicht-adhäsiven Fall.

Darüber hinaus gibt es für wachsende Tabor-Parameter einen sinkenden kritischen Wert von $\Phi$, oberhalb dessen lokales Fließen durch die adhäsive Spannungskonzentration am Rand des Kontaktes einsetzt [123].

Das Grundproblem bei der Lösung des elasto-plastischen Normalkontaktes mit Adhäsion ist die Behandlung der Spannungskonzentration am Rand des Kontaktes. In der JKRTheorie divergiert diese Spannung, es tritt dann grundsätzlich lokales Fließen am Kontaktrand auf. Tatsächlich ist die Spannung wegen der Diskretheit der atomaren Struktur

Abb.3.19 Normierte kritische Normalkraft für den adhäsiven Hertzschen Kontakt, um auf der $z$-Achse lokales Fließen zu initiieren, als Funktion des Parameters $\Phi$ (siehe Gl. (3.293)) für verschiedene Poissonzahlen; nach [123]

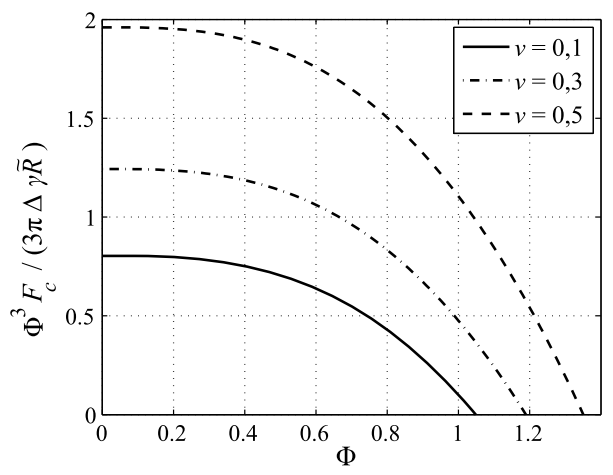


natürlich beschränkt, wie beispielsweise in der Theorie von Maugis. Trotzdem kann diese Spannung, wie oben erwähnt, ausreichen, um am Rand des Kontaktes Fließen zu initiieren. Die plastische Deformation wird dann die Öffnung und Schließung des externen Risses, den der Rand des adhäsiven Kontaktes darstellt, beeinflussen. Tvergaard und Hutchinson [124] stellten durch bruchmechanische numerische Untersuchungen fest, dass die in der inelastischen Deformation dissipierte Energie nur dann relevant gegenüber der Oberflächentrennungsarbeit wird, wenn die kohäsive (oder adhäsive) Spannung größer als die Härte des Materials ist.

\subsection{Zusammenfassung}

Dieses Kapitel widmete sich der Kontaktmechanik von axialsymmetrischen, makroskopisch glatten Körpern im Gleichgewicht. Die untersuchten Effekte, wie Reibung oder Adhäsion, wurden dabei grundsätzlich als makroskopisch gegeben betrachtet, ohne ihre tiefer liegende mikro- oder nano-tribologische Herkunft (Rauigkeit der Oberflächen auf mehreren Skalen und Anderes) genauer zu beleuchten. Alle gezeigten Modelle sind daher kontinuumsmechanischer Natur.

Eine fundamentale Voraussetzung der dargestellten Ergebnisse ist die als Halbraumnäherung bekannte Annahme, dass die Abmaße der kontaktierenden Körper sehr viel größer sind als die charakteristische Länge des Kontaktgebiets, und dass die Gradienten der sich berührenden Oberflächen (im undeformierten Zustand) in der Umgebung des Kontaktes klein sind. Wenn die Kontaktpartner außerdem elastisch ähnlich sind, kann der Kontakt zwischen ihnen auf den äquivalenten Kontakt zwischen einem starren Eindruckkörper mit einem elastischen Halbraum zurückgeführt werden.

Das reibungs- und adhäsionsfreie Normalkontaktproblem zwischen einem starren axialsymmetrischen Indenter mit einem elastischen Halbraum wird durch die Idee gelöst, dass man die Differenz zweier infinitesimal benachbarter Kontaktkonfigurationen als infinitesimale Indentierung durch einen zylindrischen Flachstempel interpretieren kann. Die Lösung des allgemeinen kreissymmetrischen Problems ergibt sich daher durch eine geeignete Superposition von Flachstempel-Lösungen.

Adhäsion spielt in Kontakten eine Rolle, wenn die betrachteten Systeme sehr klein sind, zumindest einer der Kontaktpartner sehr weich ist oder die kontaktierenden Oberflächen sehr glatt sind. Von entscheidender Bedeutung in Kontaktproblemen mit Adhäsion ist die charakteristische Reichweite der adhäsiven Wechselwirkung im Vergleich zu den Abmessungen des Kontaktes. Ist diese Reichweite klein gegenüber der kleinsten charakteristischen Kontaktlänge, spricht man vom JKR-Grenzfall des adhäsiven Kontaktes. Der entgegengesetzte Grenzfall wird durch die DMT-Theorie beschrieben. Beide Grenzfälle sind von der konkreten Form des adhäsiven Potentials unabhängig. Im Übergangsbereich zwischen beiden Grenzfällen spielt diese Form allerdings eine Rolle. Die Theorie von Maugis beschreibt den Übergang für den parabolischen Normalkontakt mithilfe des Dugdale-Stufenpotentials. 
In der JKR-Näherung ergibt sich die Lösung des axialsymmetrischen, adhäsiven Normalkontaktproblems durch die Superposition der nicht-adhäsiven Lösung mit einer StarrkörperAnhebung des Kontaktgebiets. Die Lösung des JKR-adhäsiven Kontaktproblems kann also auf die des Problems ohne Adhäsion zurückgeführt werden. Bildung und Auflösung des adhäsiven Kontaktes sind instabile Prozesse. Die kritischen Kontaktkonfigurationen hängen dabei von der Steifigkeit der Versuchsapparatur ab; die Grenzfälle sind kraft- oder weggesteuerte Versuche.

Im Gegensatz zum Normalkontaktproblem hängt die Lösung des Tangentialkontaktproblems mit Reibung nicht nur von der momentanen Kontaktkonfiguration ab, sondern im Allgemeinen von der ganzen Belastungsgeschichte, der tangential belastete Kontakt weist Gedächtnis- und Hysterese-Effekte auf. Es ist daher (ohne Kenntnis der Belastungsgeschichte) nicht möglich, einen allgemeinen Zusammenhang zwischen Tangentialkraft und -verschiebung zu formulieren. Für die einfachste Belastungsgeschichte, eine konstante Normalkraft mit einer anschließend aufgebrachten monoton wachsenden Tangentialkraft, wurde der Tangentialkontakt von Kugeln unter den folgenden Annahmen analytisch gelöst:

- Die Kontaktpartner sind elastisch ähnlich.

- Im Kontakt gilt ein lokales isotropes Amontons-Coulomb-Reibgesetz mit einem konstanten und einheitlichen Reibungskoeffizienten zwischen den kontaktierenden Oberflächen.

- Die Tangentialspannungen sind uni-direktional und rotationssymmetrisch verteilt.

- Die aus den Tangentialspannungen resultierenden Verschiebungen in Querrichtung können vernachlässigt werden.

Das Kontaktgebiet besteht dann aus einem inneren kreisförmigen Haftgebiet mit dem Radius $c \leq a$ und einem äußeren Gebiet lokalen Gleitens. Die Lösung des tangentialen Kontaktproblems ergibt sich im Rahmen der obigen (als Cattaneo-Mindlin-Näherung) bekannten Annahmen als Differenz der Lösungen des Normalkontaktproblems mit den Kontaktradien $c$ und $a$. Die Lösung für beliebige Belastungsgeschichten ergibt sich durch eine geeignete, als Algorithmus formulierbare Superposition von Lösungen für die oben beschriebene elementare Belastungsgeschichte.

Torsionskontakte weisen ähnliche Eigenschaften auf wie Tangentialkontakte: das Kontaktgebiet zerfällt in ein Haft- und Gleitgebiet und der Kontakt zeigt Gedächtnis und Hysterese. Unter den Annahmen elastischer Ähnlichkeit und lokaler Amontons-CoulombReibung kann das axialsymmetrische Kontaktproblem für die elementare Belastungsgeschichte (konstante Normalkraft und anschließend aufgebrachtes monoton wachsendes Torsionsmoment) - in Analogie zum axialsymmetrischen Normalkontakt - durch eine geeignete Superposition von Starrkörperrotationen durch einen zylindrischen Flachstempel mit wachsendem Kontaktradius $a$ gelöst werden. Die Lösung für beliebige Belastungsgeschichten ergibt sich durch eine dem Tangentialkontakt ähnliche Superposition der Lösungen für die elementare Belastungsgeschichte. 
Elastomere werden im Rahmen dieses Buches als linear-viskoelastische Medien behandelt. Das Materialverhalten kann dann durch zwei Relaxations- oder Kriechfunktionen beschrieben werden, die die Materialantwort gegen reinen Schub, bzw. hydrostatische Kompression wiedergeben. Für das reibungsfreie Normalkontaktproblem kann man den Kontakt kompressibler Medien exakt auf den mit einem äquivalenten inkompressiblen Medium zurückführen.

Zur Beschreibung der Rheologie von Elastomeren werden außerdem häufig Schaltungen von Federn und Dämpfern, sogenannte rheologische Modelle, verwendet. Das Kelvin-VoigtModell entspricht einer vollständigen Entkopplung der elastischen und viskosen Eigenschaften des Mediums und bietet deswegen die in vielerlei Hinsicht einfachste Beschreibung viskoelastischen Materialverhaltens. Weitere Modelle sind das Maxwell-Medium, das Standardmedium und Prony-Reihen mit mehreren Relaxationszeiten. Wenn das untersuchte Kontaktproblem selbst eine feste Zeitskala hat, spielt nur die Relaxation auf dieser Zeitskala eine relevante Rolle; in diesem Fall eignet sich das Kelvin-Maxwell-Modell zur Charakterisierung der viskoelastischen Eigenschaften.

Kontaktprobleme linear-viskoelastischer Medien können wegen des Korrespondenzprinzips zwischen elastischen und viskoelastischen Rand-Anfangswert-Problemen durch eine geeignete Superposition der entsprechenden elastischen Kontaktlösungen behandelt werden. Man muss dabei zwischen Belastungsgeschichten unterscheiden, bei denen der Kontaktradius monoton mit der Zeit wächst oder eine bestimmte Anzahl zeitlicher Extrema hat.

Funktionale Gradientenmedien (FGM) können gegenüber homogenen Medien in vielen Fällen deutlich verbesserte mechanische Eigenschaften aufweisen. Eine geschlossen analytische Behandlung von FGM ist nur für sehr spezielle Formen der Inhomogenität möglich, beispielsweise, wenn der elastische Modul mit der Tiefe nach einem Potenzgesetz variiert. Wie im Fall homogener Materialien kann man für FGM das axialsymmetrische nicht-adhäsive Normalkontaktproblem durch die Superposition infinitesimaler Flachstempel-Indentierungen lösen. Adhäsive Normalkontakte und Tangentialkontakte mit Reibung kann man mit den anhand homogener Medien entwickelten Methoden auf den nicht-adhäsiven Normalkontakt zurückführen.

Die Spannungen in mechanischen Kontakten sind so groß und räumlich konzentriert, dass es leicht zu lokalem Fließen der kontaktierenden Körper kommen kann. Ein vollständiger Belastungszyklus eines elasto-plastischen Kontaktes besteht dabei aus vier Stadien: elastische, elasto-plastische und voll-plastische Belastung sowie elastische Entlastung mit einem durch die plastische Deformation veränderten Profil. Die rigorose Behandlung des elasto-plastischen Normalkontaktproblems ist in der Regel nur mithilfe der FEM möglich. Unter bestimmten Annahmen können aber analytische Näherungslösungen gefunden werden. Adhäsion reduziert die nötige äußere Normalkraft, um in einem Kontakt plastische Deformationen zu initiieren. 


\section{Literatur}

1. Boussinesq, J. (1885). Application des Potentiels a L'etude de L'Equilibre et du Mouvement des Solides Elastiques. Lille: Imprimerie L. Danel.

2. Cerruti, V. (1882). Ricerche intorno all'equilibrio de' corpi elastici isotropi. Rendiconte della Accademia Nazionale dei Lincei, 3(13), 81-122.

3. Landau, L. D., \& Lifshitz, E. M. (1975). Lehrbuch der Theoretischen Physik, Bd. VII: Elastizitätstheorie. Berlin: Akademie.

4. Rao, A. K. (1971). Stress Concentrations and Singularities at Interface Corners. ZAMM Zeitschrift für Angewandte Mathematik und Mechanik, 51(5), 395-406.

5. Föppl, L. (1941). Elastische Beanspruchung des Erdbodens unter Fundamenten. Forschung auf dem Gebiet des Ingenieurwesens A, 12(1), 31-39.

6. Schubert, G. (1942). Zur Frage der Druckverteilung unter elastisch gelagerten Tragwerken. Ingenieur-Archiv, 13(3), 132-147.

7. Galin, L. A. (1946). Spatial contact problems of the theory of elasticity for punches of circular shape in plane. PrikladnayaMatematika i Mekhanika, 10, 425-448.

8. Sneddon, I. N. (1965). The relation between load and penetration in the axisymmetric boussinesq problem for a punch of arbitrary profile. International Journal of Engineering Science, 3(1), 47-57.

9. Mossakovski, V. I. (1963). Compression of elastic bodies under conditions of sticking (axisymmetric case). PMM Journal of Applied Mathematics and Mechanics, 27(3), 630-643.

10. Jäger, J. (1995). Axi-symmetric bodies of equal material under torsion or shift. Archive of Applied Mechanics, 65(7), 478-487.

11. Johnson, K. L. (1985). Contact mechanics. Cambridge: Cambridge University Press.

12. Popov, V. L. (2015). Kontaktmechanik und Reibung. Von der Nanotribologie bis zur Erdbebendynamik, 3. Aufl. Berlin: Springer-Vieweg.

13. Barber, J. R. (2018). Contact mechanics. Basel: Springer.

14. Bracewell, R. N. (2000). The fourier transform and its applications (3. Aufl.). New York: McGraw-Hill Book Company.

15. Hertz, H. (1882). Über die Berührung fester elastischer Körper. Journal für die reine und angewandte Mathematik, 92, 156-171.

16. Segedin, C. M. (1957). The relation between load and penetration for a spherical punch. Mathematika, 4(2), 156-161.

17. Popov, V. L., Heß, M., \& Willert, E. (2018). Handbuch der Kontaktmechanik. Exakte Lösungen axialsymmetrischer Kontaktprobleme. Berlin: Springer Vieweg.

18. Mossakovski, V. I. (1954). The fundamental mixed problem of the theory of elasticity for a half-space with a circular line separating the boundary conditions. Prikladnaya Matematika $i$ Mekhanika, 18, 187-196.

19. Spence, D. A. (1968). Self-similar solutions to adhesive contact problems with incremental loading. Proceedings of the Royal Society of London, Series A, 305, 55-80.

20. Spence, D. A. (1975). The hertz contact problem with finite friction. Journal of Elasticity, 5(3-4), 297-319.

21. Storåkers, B., \& Elaguine, D. (2005). Hertz contact at finite friction and arbitrary profiles. Journal of the Mechanics and Physics of Solids, 53(6), 1422-1447.

22. Zhupanska, O. I. (2009). Axisymmetric contact with friction of a rigid spherewith an elastic half-space. Proceedings of the Royal Society of London, Series A, 465, 2565-2588.

23. Borodich, F. M., \& Keer, L. M. (2004). Contact problems and depth-sensing nanoindentation for frictionless and frictional boundary conditions. International Journal of Solids and Structures, 41(9-10), 2479-2499. 
24. Griffith, A. A. (1921). The phenomena of rupture and flow in solids. Philosophical Transactions of the Royal Society of London, Series A, 221, 163-198.

25. Johnson, K. L., Kendall, K., \& Roberts, A. D. (1971). Surface energy and the contact of elastic solids. Proceedings of the Royal Society of London, Series A, 324, 301-313.

26. Derjaguin, B. V., Muller, V. M., \& Toporov, Y. P. (1975). Effect of contact deformations on the adhesion of particles. Journal of Colloid and Interface Science, 53(2), 314-326.

27. Bradley, A. I. (1932). The cohesive force between solid surfaces and the surface energy of solids. The London, Edinburgh, and Dublin Philosophical Magazine and Journal of Science, 13(86), 853-862.

28. Tabor, D. (1977). Surface forces and surface interactions. Journal of Colloid and Interface Science, 58(1), 2-13.

29. Maugis, D. (1992). Adhesion of spheres: The JKR-DMT transition using a Dugdale model. Journal of Colloid and Interface Science, 150(1), 243-269.

30. Dugdale, D. S. (1960). Yielding of steel sheets containing slits. Journal of the Mechanics and Physics of Solids, 8(2), 100-104.

31. Greenwood, J. A. (1997). Adhesion of elastic spheres. Proceedings of the Royal Society of London, Series A, 453, 1277-1297.

32. Feng, J. Q. (2000). Contact behavior of spherical elastic particles: A computational study of particle adhesion and deformations. Colloids and Surfaces A: Physicochemical and Engineering Aspects, 172(1-3), 175-198.

33. Muller, V. M., Yushchenko, V. S., \& Derjaguin, B. V. (1980). On the influence of molecular forces on the deformation of an elastic sphere and its sticking to a rigid plane. Journal of Colloid and Interface Science, 77(1), 91-101.

34. Greenwood, J. A., \& Johnson, K. L. (1998). An alternative to the Maugis model of adhesion between elastic spheres. Journal of Physics D: Applied Physics, 31(22), 3279-3290.

35. Barthel, E. (1998). On the Description of the Adhesive Contact of Spheres with Arbitrary Interaction Potentials. Journal of Colloid and Interface Science, 200(1), 7-18.

36. Ciavarella, M., Joe, J., Papangelo, A., \& Barber, J. R. (2019). The role of adhesion in contact mechanics. Journal of the Royal Society Interface, 16, 20180738. https://doi.org/10.1098/rsif. 2018.0738 .

37. Barquins, M., \& Maugis, D. (1982). Adhesive contact of axisymmetric punches on an elastic half-space: The modified Hertz-Huber's stress tensor for contacting spheres. Journal de Mécanique Théorique et Appliquée, 1(2), 331-357.

38. Kesari, H., \& Lew, A. J. (2012). Adhesive frictionless contact between an elastic isotropic half-space and a rigid axi-symmetric punch. Journal of Elasticity, 106(2), 203-224.

39. Argatov, I. I., Li, Q., Pohrt, R., \& Popov, V. L. (2016). Johnson-Kendall-Roberts adhesive contact for a toroidal indenter. Proceedings of the Royal Society of London, Series A, 472, 20160218. https://doi.org/10.1098/rspa.2016.0218.

40. Popov, V. L. (2018). Solution of adhesive contact problem on the basis of the known solution for nonadhesive one. Facta Universitatis, Series Mechanical Engineering, 16(1), 93-98.

41. Ciavarella, M. (2018). An approximate JKR solution for a general contact, including rough contacts. Journal of the Mechanics and Physics of Solids, 114, 209-218.

42. Popov, V. L. (2017). Surface profiles with zero and finite adhesion force and adhesion instabilities. https://arxiv.org/abs/1707.07867.

43. Giannakopoulos, E. A., Lindley, T. C., \& Suresh, S. (1998). Aspects of equivalence between contact mechanics and fracture mechanics: Theoretical connections and a life-prediction methodology for fretting-fatigue. Acta Materialia, 46(9), 2955-2968.

44. Savkoor, A. R., \& Briggs, G. A. D. (1977). The effect of tangential force on the contact of elastic solids in adhesion. Proceedings of the Royal Society of London, Series A, 356, 103-114. 
45. Hutchinson, J. W. (1990). Mixed mode fracture mechanics of interfaces. In M. von Rühle (Hrsg.), Metal-ceramic interfaces, (S. 295-306). Pergamon.

46. Johnson, K. L. (1997). Adhesion and friction between a smooth elastic spherical asperity and a plane surface. Proceedings of the Royal Society of London, Series A, 453, 63-179.

47. Popov, V. L., Lyashenko, I. A., \& Filippov, A. E. (2017). Influence of tangential displacement on the adhesion strength of a contact between a parabolic profile and an elastic half-space. Royal Society Open Science, 4(8), 161010. https://doi.org/10.1098/rsos.161010.

48. Kim, K. S., McMeeking, R. M., \& Johnson, K. L. (1998). Adhesion, slip, cohesive zones and energy fluxes for elastic spheres in contact. Journal of the Mechanics and Physics of Solids, 46(2), 243-266.

49. Waters, J. F., \& Guduru, P. R. (2009). Mode-mixity-dependent adhesive contact of a sphere on a plane surface. Proceedings of the Royal Society of London, Series A, 466, 1303-1325.

50. Menga, N., Carbone, G., \& Dini, D. (2018). Do uniform tangential interfacial stresses enhance adhesion? Journal of the Mechanics and Physics of Solids, 112, 145-156.

51. Popov, V. L., \& Dimaki, A. V. (2017). Friction in an adhesive tangential contact in the CoulombDugdale approximation. The Journal of Adhesion, 93(14), 1131-1145.

52. Filippov, A. E., Klafter, J., \& Urbakh, M. (2004). Friction through dynamical formation and rupture of molecular bonds. Physical Review Letters, 92(13), 135503. https://doi.org/10.1103/ PhysRevLett.92.135503.

53. Borodich, F. M., Galanov, B. A., Prostov, Y. I., \& Suarez-Alvarez, M. M. (2012). Influence of complete sticking on the indentation of a rigid cone into an elastic half-space in the presence of molecular adhesion. PMM Journal of Applied Mathematics and Mechanics, 76(5), 590-596.

54. Lyashenko, I. A., Willert, E., \& Popov, V. L. (2016). Adhesive impact of an elastic sphere with an elastic half space: Numerical analysis based on the method of dimensionality reduction. Mechanics of Materials, 92, 155-163.

55. Mergel, J. C., Sahli, R., Scheibert, J., \& Sauer, R. A. (2018). Continuum contact models for coupled adhesion and friction. The Journal of Adhesion, 95(12), 1101-1133. https://doi.org/ 10.1080/00218464.2018.1479258.

56. Cattaneo, C. (1938). Sul Contatto di due Corpore Elastici: Distribuzione degli sforzi. Rendiconti dell' Academi Nazionale dei Lincei, 27, 342-348, 434-436, 474-478.

57. Mindlin, R. D. (1949). Compliance of Elastic Bodies in Contact. Journal of Applied Mechanics, 16(3), 259-268.

58. Munisamy, R. L., Hills, D. A., \& Nowell, D. (1994). Static Axisymmetric Hertzian contacts subject to shearing forces. Journal of Applied Mechanics, 61(2), 278-283.

59. Mindlin, R. D., \& Deresiewicz, H. (1953). Elastic spheres in contact under varying oblique forces. Journal of Applied Mechanics, 20(3), 327-344.

60. Jäger, J. (1993). Elastic contact of equal spheres under oblique forces. Archive of Applied Mechanics, 63(6), 402-412.

61. Jäger, J. (1998). A new principle in contact mechanics. Journal of Tribology, 120(4), 677-684.

62. Ciavarella, M. (1998). Tangential loading of general three-dimensional contacts. Journal of Applied Mechanics, 65(4), 998-1003.

63. Ciavarella, M. (1998). The generalized Cattaneo partial slip plane contact problem. I - Theory. International Journal of Solids and Structures, 35(18), 2349-2362.

64. Aleshin, V., \& Van Den Abeele, K. (2012). Hertz-Mindlin problem for arbitrary oblique 2D loading: General solution by memory diagrams. Journal of the Mechanics and Physics of Solids, 60(1), 14-36.

65. Aleshin, V., Bou Matar, O., \& Van Den Abeele, K. (2015). Method of memory diagrams for mechanical frictional contacts subject to arbitrary 2D loading. International Journal of Solids and Structures, 60-61, 84-95. 
66. Lubkin, J. L. (1951). The torsion of elastic spheres in contact. Journal of Applied Mechanics, $18(2), 183-187$.

67. Jäger, J. (1994). Torsional impact of elastic spheres. Archive of Applied Mechanics, 64(4), 235-248.

68. Deresiewicz, H. (1954). Contact of elastic spheres under an oscillating torsional couple. Journal of Applied Mechanics, 21, 52-56.

69. Vandamme, M., \& Ulm, F. J. (2006). Viscoelastic solutions for conical indentation. International Journal of Solids and Structures, 43(10), 3142-3165.

70. Zimmermann, J., \& Stommel, M. (2013). The mechanical behaviour of rubber under hydrostatic compression and the effect on the results of finite element analyses. Archive of Applied Mechanics, 83(2), 293-302.

71. Bachrach, N. M., Mow, V. C., \& Guilak, F. (1998). Incompressibility of the solid matrix of articular cartilage under high hydrostatic pressures. Journal of Biomechanics, 31(5), 445-451.

72. Tanaka, Y., Klemann, V., Martinec, Z., \& Riva, R. E. M. (2011). Spectral-finite element approach to viscoelastic relaxation in a spherical compressible Earth: Application to GIA modelling. Geophysical Journal International, 184(1), 220-234.

73. Alfrey, T. (1944). Non-homogeneous stresses in visco-elasticmedia. Quarterly of Applied Mathematics, 2(2), 113-119.

74. Lee, E. H. (1955). Stress analysis in visco-elastic bodies. Quarterly of Applied Mathematics, 13(2), 183-190.

75. Radok, J. R. M. (1957). Visco-elastic stress analysis. Quarterly of Applied Mathematics, 15(2), 198-202.

76. Willert, E. (2018). Short note: Method of dimensionality reduction for compressible viscoelasticmedia. II. Frictionless normal contact for arbitrary shear and bulk rheologies. ZAMM Zeitschrift für Angewandte Mathematik und Mechanik, 98(11), 2022-2026.

77. Lee, E. H., \& Radok, J. R. M. (1960). The contact problem for viscoelastic bodies. Journal of Applied Mechanics, 27(3), 438-444.

78. Graham, G. A. C. (1965). The contact problem in the linear theory of viscoelasticity. International Journal of Engineering Science, 3(1), 27-46.

79. Ting, T. C. T. (1966). The contact stresses between a rigid indenter and a viscoelastic half-space. Journal of Applied Mechanics, 33(4), 845-854.

80. Hunter, S. C. (1960). The Hertz problem for a rigid spherical indenter and a viscoelastic halfspace. Journal of the Mechanics and Physics of Solids, 8(4), 219-234.

81. Greenwood, J. A. (2010). Contact between an axisymmetric indenter and a viscoelastic halfspace. International Journal of Mechanical Sciences, 52(6), 829-835.

82. Graham, G. A. C. (1967). The contact problemin the linear theory of viscoelasticitywhen the time dependent contact area has any number of maxima and minima. International Journal of Engineering Science, 5(6), 495-514.

83. Ting, T. C. T. (1968). Contact problems in the linear theory of viscoelasticity. Journal of Applied Mechanics, 35(2), 248-254.

84. Liu, Z., Meyers, M. A., Zhang, Z., \& Ritchie, R. O. (2017). Functional gradients and heterogeneities in biological materials: Design principles, functions, and bioinspired applications. Progress in Materials Science, 88, 467-498.

85. Suresh, S. (2001). Graded Materials for Resistance to Contact Deformation and Damage. Science, 292, 2447-2451.

86. Holl, D. L. (1941). Stress transmissions in earths. Highway Research Board Proceedings, 20, 709-721.

87. Rostovtsev, N. A. (1961). An integral equation encountered in the problem of a rigid foundation bearing on nonhomogeneous soil. PMM Journal of Applied Mathematics and Mechanics, 25(1), 238-246. 
88. Gibson, R. E. (1967). Some results concerning displacements and stresses in a nonhomogeneous elastic half-space. Géotechnique, 17(1), 58-67.

89. Booker, J. R., Balaam,N. P., \& Davis, E. H. (1985). The behaviour of an elastic nonhomogeneous half-space. Part I-line and point loads. International Journal for Numerical and Analytical Methods in Geomechanics, 9(4), 353-367.

90. Booker, J. R., Balaam,N. P., \& Davis, E. H. (1985). The behaviour of an elastic nonhomogeneous half-space. Part II-circular and strip footings. International Journal for Numerical and Analytical Methods in Geomechanics, 9(4), 369-381.

91. Giannakopoulos, E. A., \& Suresh, S. (1997). Indentation of solids with gradients in elastic properties: Part I. Point force. International Journal of Solids and Structures, 34(19), 23572392.

92. Giannakopoulos, E. A., \& Suresh, S. (1997). Indentation of solids with gradients in elastic properties: Part II. axisymmetric indentors. International Journal of Solids and Structures, 34(19), 2393-2428.

93. Selvadurai, A. P. (2007). The analytical method in geomechanics. Applied Mechanics Reviews, 60(3), 87-106.

94. Fabrikant, V. I., \& Sankar, T. S. (1984). On contact problems in an inhomogeneous half-space. International Journal of Solids and Structures, 20(2), 159-166.

95. Chen, S., Yan, C., Zhang, P., \& Gao, H. (2009). Mechanics of adhesive contact on a power-law graded elastic half-space. Journal of the Mechanics and Physics of Solids, 57(9), 1437-1448.

96. Jin, F., \& Guo, X. (2013). Mechanics of axisymmetric adhesive contact of rough surfaces involving powerlaw graded materials. International Journal of Solids and Structures, 50(2021), 3375-3386.

97. Jin, F., Guo, X., \& Zhang, W. (2013). A unified treatment of axisymmetric adhesive contact on a power-law graded elastic half-space. Journal of Applied Mechanics, 80(6), 061024. https:// doi.org/10.1115/1.4023980.

98. Heß, M., \& Popov, V. L. (2016). Method of dimensionality reduction in contact mechanics and friction: A user's handbook. II. Power-law graded materials. Facta Universitatis, Series Mechanical Engineering, 14(3), 251-268.

99. Paulino, G. H., \& Jin, Z. H. (2001). Correspondence principle in viscoelastic functionally graded materials. Journal of Applied Mechanics, 68(1), 129-132.

100. Willert, E. (2018). Dugdale-Maugis adhesive normal contact of axisymmetric power-law graded elastic bodies. Facta Universitatis, Series Mechanical Engineering, 16(1), 9-16.

101. Willert, E., \& Popov, V. L. (2017). The oblique impact of a rigid sphere on a power-law graded elastic halfspace. Mechanics of Materials, 109, 82-89.

102. Etsion, I., Kligerman, Y., \& Kadin, Y. (2005). Unloading of an elastic-plastic loaded spherical contact. International Journal of Solids and Structures, 42(13), 3716-3729.

103. Ghaednia, H., et al. (2017). A review of elastic-plastic contact mechanics. AppliedMechanics Review, 69(6), 060804. https://doi.org/10.1115/1.4038187.

104. Ishlinski, A. J. (1944). The problem of plasticity with the axial symmetry and Brinell's test. Prikladnaya Matematika i Mekhanika, 8, 201-224.

105. Tabor, D. (1951). The Hardness of Metals. Oxford: Oxford University Press.

106. Hardy, C., Baronet, C. N., \& Tordion, G. V. (1971). The elasto-plastic indentation of a half-space by a rigid sphere. International Journal for Numerical Methods in Engineering, 3(4), 451-462.

107. Follansbee, P. S., \& Sinclair, G. B. (1984). Quasi-static normal indentation of an elasto-plastic half-space by a rigid sphere - I: Analysis. International Journal of Solids and Structures, 20(1), 81-91.

108. Sinclair, G. B., Follansbee, P. S., \& Johnson, K. L. (1985). Quasi-static normal indentation of an elasto-plastic half-space by a rigid sphere - II: Results. International Journal of Solids and Structures, 21(8), 865-888. 
109. Hill, R., Storåkers, B., \& Zdunek, A. B. (1989). A theoretical study of the Brinell hardness test. Proceedings of the Royal Society of London, Series A, 423, 301-330.

110. Mesarovic, S. D., \& Fleck, N. A. (1999). Spherical indentation of elastic-plastic solids. Proceedings of the Royal Society of London, Series A, 455, 2707-2728.

111. Mesarovic, S. D., \& Fleck, N. A. (2000). Frictionless indentation of dissimilar elastic-plastic spheres. International Journal of Solids and Structures, 37(46-47), 7071-7091.

112. Ghaednia, H., Pope, S. A., Jackson, R. L., \& Marghitu, D. B. (2016). A comprehensive study of the elastoplastic contact of a sphere and a flat. Tribology International, 93(A), 78-90.

113. Kogut, L., \& Etsion, I. (2002). Elastic-plastic contact analysis of a sphere and a rigid flat. Journal of Applied Mechanics, 69(5), 657-662.

114. Jackson, R. L., \& Kogut, L. (2005). A comparison of flattening and indentation approaches for contact mechanics modeling of single asperity contacts. Journal of Tribology, 128(1), 209-212.

115. Vu-Quoc, L., Zhang, X., \& Lesburg, L. (1999). A normal force-displacement model for contacting spheres accounting for plastic deformation: Force-driven formulation. Journal of Applied Mechanics, 67(2), 363-371.

116. Thornton, C. (1997). Coefficient of restitution for collinear collisions of elastic-perfectly plastic spheres. Journal of Applied Mechanics, 64(2), 383-386.

117. Zhao, Y., Maietta, D. M., \& Chang, L. (2000). An asperity microcontact model incorporating the transition from elastic deformation to fully plastic flow. Journal of Tribology, 122(1), 86-93.

118. Brake, M. R. (2012). An analytical elastic-perfectly plastic contact model. International Journal of Solids and Structures, 49(22), 3129-3141.

119. Huber, M. T. (1904). Zur Theorie der Berührung fester elastischer Körper. Annalen der Physik, $14,153-163$.

120. Li, L. Y., Wu, C. Y., \& Thornton, C. (2001). A theoretical model for the contact of elastoplastic bodies. Proceedings of the Institution of Mechanical Engineers, Part C: Journal of Mechanical Engineering Science, 216(4), 421-431.

121. Jackson, R. L., Green, I., \& Marghitu, D. B. (2010). Predicting the coefficient of restitution of impacting elastic-perfectly plastic spheres. Nonlinear Dynamics, 60(3), 217-229.

122. Mesarovic, S. D., \& Johnson, K. L. (2000). Adhesive contact of elastic-plastic spheres. Journal of the Mechanics and Physics of Solids, 48(10), 2009-2033.

123. Wu, Y. C., \& Adams, G. G. (2008). Plastic yield conditions for adhesive contacts between a rigid sphere and an elastic half-space. Journal of Tribology, 131(1), 011403. https://doi.org/10. $1115 / 1.3002329$.

124. Tvergaard, V., \& Hutchinson, J. W. (1992). The relation between crack growth resistance and fracture process parameters in elastic-plastic solids. Journal of the Mechanics and Physics of Solids, 40(6), 1377-1397. 
Open Access Dieses Kapitel wird unter der Creative Commons Namensnennung 4.0 International Lizenz (http://creativecommons.org/licenses/by/4.0/deed.de) veröffentlicht, welche die Nutzung, Vervielfältigung, Bearbeitung, Verbreitung und Wiedergabe in jeglichem Medium und Format erlaubt, sofern Sie den/die ursprünglichen Autor(en) und die Quelle ordnungsgemäß nennen, einen Link zur Creative Commons Lizenz beifügen und angeben, ob Änderungen vorgenommen wurden.

Die in diesem Kapitel enthaltenen Bilder und sonstiges Drittmaterial unterliegen ebenfalls der genannten Creative Commons Lizenz, sofern sich aus der Abbildungslegende nichts anderes ergibt. Sofern das betreffende Material nicht unter der genannten Creative Commons Lizenz steht und die betreffende Handlung nicht nach gesetzlichen Vorschriften erlaubt ist, ist für die oben aufgeführten Weiterverwendungen des Materials die Einwilligung des jeweiligen Rechteinhabers einzuholen. 